\title{
Authenticated Encryption in the Face of Protocol and Side Channel Leakage $^{\star}$
}

\author{
Guy Barwell ${ }^{1}$, Daniel P. Martin ${ }^{2}$, Elisabeth Oswald ${ }^{1}$, and Martijn Stam ${ }^{1}$ \\ 1 Department of Computer Science, University of Bristol, \\ Merchant Venturers Building, Woodland Road, \\ Bristol, BS8 1UB, United Kingdom. \\ rgb.crypto@gmail.com, \{elisabeth.oswald, martijn.stam\}@bris.ac.uk \\ 2 School of Mathematics, University of Bristol, Bristol, BS8 1TW, UK, \\ and the Heilbronn Institute for Mathematical Research, Bristol, UK. \\ dan.martin@bris.ac.uk
}

\begin{abstract}
Authenticated encryption schemes in practice have to be robust against adversaries that have access to various types of leakage, for instance decryption leakage on invalid ciphertexts (protocol leakage), or leakage on the underlying primitives (side channel leakage). This work includes several novel contributions: we augment the notion of nonce-base authenticated encryption with the notion of continuous leakage and we prove composition results in the face of protocol and side channel leakage. Moreover, we show how to achieve authenticated encryption that is simultaneously both misuse resistant and leakage resilient, based on a sufficiently leakage resilient PRF, and finally we propose a concrete, pairing-based instantiation of the latter.
\end{abstract}

Keywords: provable security, authenticated encryption, generic composition, leakage resilience, robustness

${ }^{\star}$ An extended abstract of this paper will appear at ASIACRYPT 2017; this is the full version. 


\section{Table of Contents}

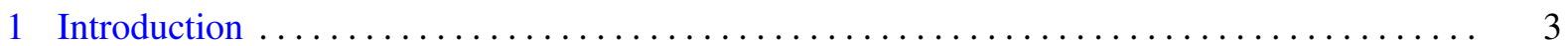

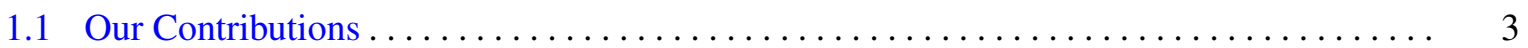

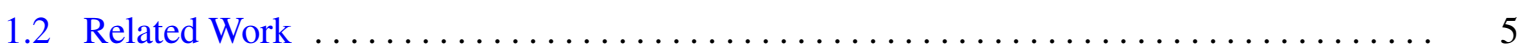

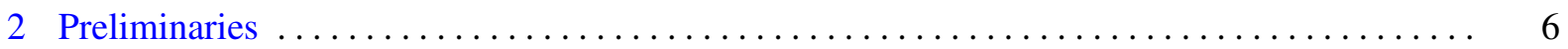

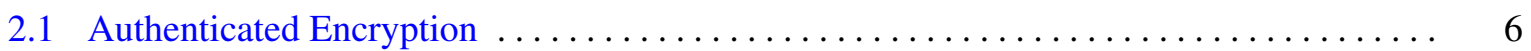

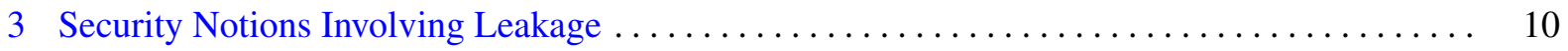

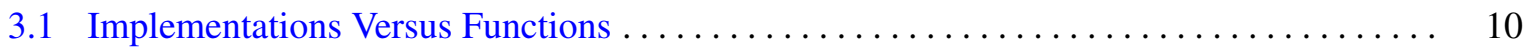

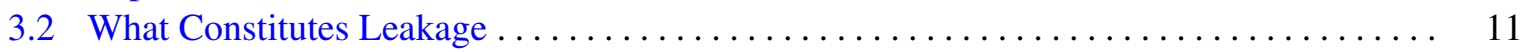

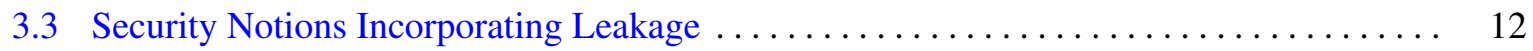

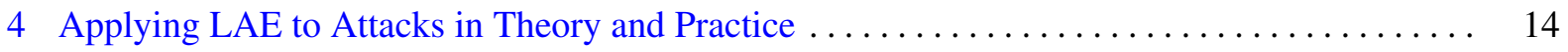

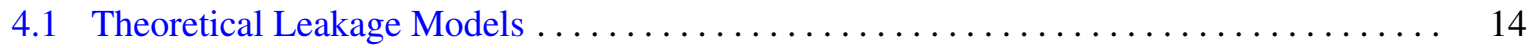

4.2 Casting an Existing Attack against GCM into Our Framework ................ 15

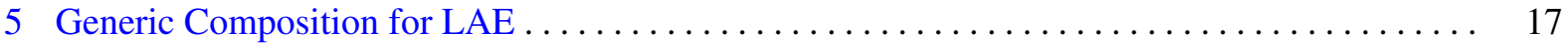

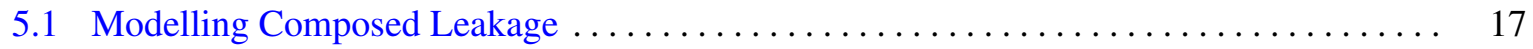

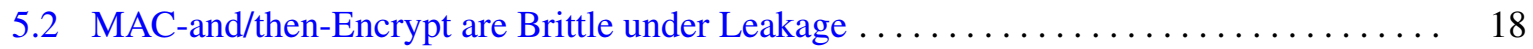

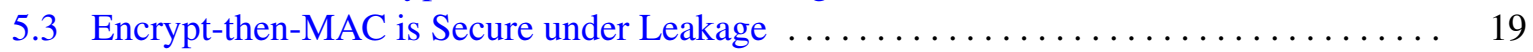

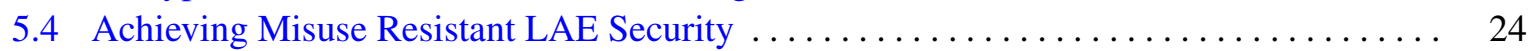

5.5 A Leakage Resilient IV-based Encryption Scheme . . . . . . . . . . . . . . . . . . . . . . 25

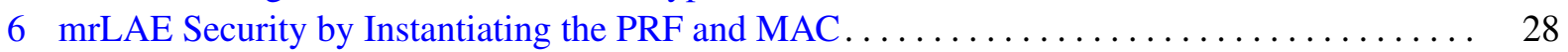

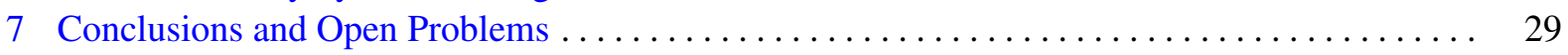

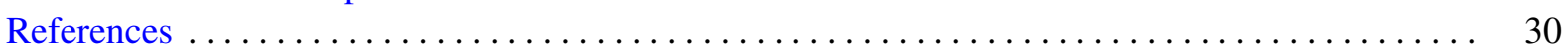

A A PRF and MAC Resilient Against Continuous and Fully Adaptive Leakage $\ldots \ldots \ldots \ldots \ldots . \ldots 32$

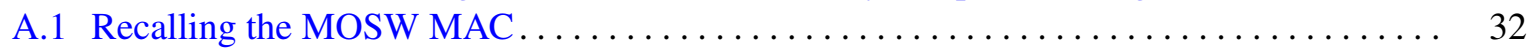

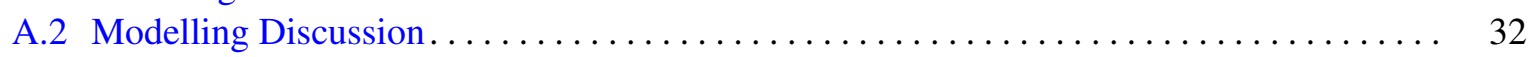

A.3 A Leakage Resilient PRF ...................................... 33

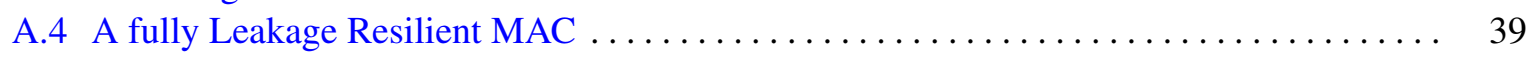

A.5 nLAE and mrLAE Constructions in the Generic Group Model................. 41 


\section{Introduction}

Authenticated Encryption (AE) has arisen out of (practical) necessity: historic modes-of-operation for symmetric encryption [42] implicitly target confidentiality against passive adversaries, but most realistic threat models also demand security against active adversaries. Thwarting adversaries trying to modify ciphertexts is best captured by requiring ciphertext integrity; encryption schemes that offer both this and a suitable passive indistinguishability notion are said to provide authenticated encryption. Today, authenticated encryption has become the primitive of choice to enable secure communication. AE schemes can be constructed from components that individually provide either confidentiality or authenticity, both in a traditional probabilistic setting [8] and a more modern nonce-based one [40]. As a result, there exist several black-box constructions of authenticated encryption schemes based on simpler, keyed primitives such as pseudorandom functions or permutations, including MACs and blockciphers.

Unfortunately, in practice neither the composition nor the underlying components behave as blackboxes: side-channel attacks often leak additional information to an adversary, leading to real-life breaks (e.g. [57]). Invariably, these attacks are possible by exploiting a discrepancy between the capabilities of a theoretical adversary and an actual, real-life one. Thus, these attacks neither violate the security assumptions on the primitive nor do they invalidate the security claims: rather, they render these claims insufficient and the existing security models as inadequate.

In response, a number of works have tried to capture more closely how protocols behave when implemented [14,21,25]. We are particularly interested in subtle authenticated encryption [4] which augments the authenticated encryption security game with an implementation-dependent leakage oracle that provides an adversary deterministic decryption leakage on invalid ciphertexts only. Subtle authenticated encryption encompasses earlier notions such as multiple decryption errors [13] and the release of unverified plaintexts [2]; it can be regarded as protocol leakage.

Orthogonally, primitives can leak. Kocher (et al.) [30,31] showed how both timing and power measurements lead to a side-channel, enabling the extraction of secret data out of cryptographic devices. Primitives believed to be secure, such as AES, were broken without actually violating the assumption that AES is a secure pseudorandom permutation. Such attacks are captured in the framework of leakage resilient cryptography. Here an adversary can adaptively choose a leakage function that is restricted in scope as only computation is assumed to leak information [39], and in size. The latter is captured by leaking only a certain number of bits per call. If the overall leakage remains unbounded the model is referred to as continuous leakage. For a variety of schemes and security notions, resilience against certain classes of leakage can be proven [17,29,56], but dealing with adaptivity that allows leakage after an adversary has received a challenge is often problematic.

The current theory of authenticated encryption is not suited to take this additional leakage resource into account. In this work we provide a framework for dealing with AE in the presence of leakage, which then allows us to determine the constraints on primitives and constructions alike to yield $\mathrm{AE}$ secure against classes of leakage functions. Moreover, we propose a concrete instantiation of a leakage-resilient pseudorandom function suitable to be used to form the first leakage-resilient, nonce-based authenticated encryption scheme.

\subsection{Our Contributions}

Augmenting nonce-base authenticated encryption with leakage. We start by augmenting the noncebased authenticated encryption security notion (Section 2.1) with leakage (Section 3). This new notion, which we will refer to as LAE, can be regarded as a generalization of the SAE framework by Barwell et al. [4], yet it also captures leakage-resilience as introduced by Dziembowski and Pietrzak [19]. We provide corresponding leakage notions for the primitives used by the composition results by Namprempre, Rogaway and Shrimpton [40] (henceforth NRS), namely nonce- or iv-based encryption, pseudorandom functions, and message authentication codes.

For the traditional AE notion by Rogaway and Shrimpton [51], an adversary has to distinguish between a world with a real encryption and decryption oracle on the one hand, and a world with a 
random ciphertext generator and a rejection oracle on the other. In the LAE game the number of oracles available to the adversary increased from two to four: both worlds are augmented with true encryption and decryption oracles and we will allow (only) these additional oracles to leak.

For the leakage mechanism, we adopt the approach originally suggested by Micali and Reyzin [39] and later adapted for leakage resilience [19] where an adversary can provide a leakage function to be evaluated on the internal variables of the oracle, with the leakage output to be returned to the adversary alongside the normal output. The model is very powerful, allowing the adversary to adaptively choose which leakage function they would like evaluated on a query by query basis.

To avoid trivial wins, the leakage functions that are allowed need to be restricted to prevent, for instance, leaking the entire key in one go. We model this by explicitly defining security relative to a class of leakage functions (as is common for instance in the contexts for related-key or key-dependent message attacks). By appropriately setting the class of leakage functions, we show that our notion generalises previous strengthened AE security notions, including SAE, RUP and distinguishable decryption errors $[2,4,13]$, and previous leakage notions, including the simulatable leakage, auxiliary input and probing models $[17,26,56]$.

Generic composition with leakage. Our second contribution (Section 5) is an investigation on how to perform generic composition in the presence of leakage by extending the results of NRS [40]. We establish that schemes susceptible to release of unverified plaintext are unsuitable even for much more modest types of leakage and we confirm modern folklore that this affects all schemes that are roughly of the type Encrypt-and-MAC or MAC-then-Encrypt (cf. [2]). Conversely, we show that Encrypt-thenMAC style schemes are secure against a large class of leakage functions, where we express this class in terms of the leakage classes against which the underlying primitives are secure. For this composition of leakage from different primitives, we effectively just concatenate the leakage of the constituent parts, which implicitly assumes that only computation leaks (cf. [39]).

In particular, we show security of the N2 and A5 constructions of NRS against nonce-respecting adversaries (Theorem 1 and Corollary 1), and of A6 against adversaries who never repeat a nonce and associated-data pair (Corollary 2).

The above result imply that none of the NRS schemes achieve misuse resistant LAE security (mrLAE), hence we propose a novel generic construction that does meet this strongest definition of security, albeit at the cost of further ciphertext expansion (Theorem 3). Our result gives ciphertexts that are two blocks longer than the messages (rather than the single block expansion of an NRS scheme): we leave open whether mrLAE security can be achieved with less ciphertext expansion.

Moreover, we show that instantiating CFB mode with a pseudorandom function yields a secure ivbased encryption scheme even under leakage (Theorem 4). This allows us to apply our generic composition results to construct the first $\mathrm{AE}$ scheme secure against continuous leakage based on a pseudorandom function actively secure against continuous leakage and a MAC scheme secure against continuous leakage of both tagging and verification.

Instantiation using a new leakage resilient PRF. Our final contribution (Appendix A) is the construction of these latter two primitives. To this end, we extend the MAC of Martin et al. [36] in two directions. First, we show how it can be adapted such that it may leak under verification(Theorem 9), answering an open question from their work. Then, we show how to implement the tagging function such that it is a PRF in the face of leakage. While the previous implementation of the MAC is a pseudorandom function when no leakage is present, already small amounts of leakage are disastrous for the pseudorandomness property. It turns out that the underlying key update mechanism due to Kiltz and Pietrzak [29] is intrinsically unsuitable to create an actively secure pseudorandom function: the mechanism shares a key out in two which allows a form of leak-in-the-middle attack. The solution we propose is to use three shares instead and we prove that the resulting construction is indeed a pseudorandom function that is leakage-resilient even against adaptive adversaries. 


\subsection{Related Work}

Authenticated encryption. One of the earliest symmetric works on concrete security of AE was by Bellare and Namprempre [8]. Working within the probabilistic model, they formalised what it meant to be both confidential and authentic, and investigated how one could achieve this through generic composition, combining two schemes (one with each security property) such that their composition achieved both. Yet, modern authenticated encryption is a stateless and deterministic notion, taking in any randomness or state as an extra parameter termed the nonce. It was formalised across a number of papers, culminating in Rogaway and Shrimpton's 2006 work on DAE [51] and only recently a comprehensive study of all the ways one could combine a PRF with an encryption scheme was completed in the noncebased setting [40].

The CAESAR competition [10] has driven further research into AE, and particularly into the concept of robustness, namely the idea that a scheme should be more resistant to common problems faced in the real-world. One branch of this research has been into designing schemes that are resistant to certain forms of leakage. Prior to the competition, Boldyreva et al. [13] had investigated how to model a scheme from which decryption failures are not identical, such as under a timing attack. Andreeva et al. [2] (RUP) considered the release of unverified plaintexts, where the decryption oracle releases candidate plaintexts even if they fail verification. The robust authenticated encryption notion of Hoang et al. [25] also implies security against the leakage of these candidate plaintexts, among other goals. Barwell et al. [4] defined the SAE framework as a generalisation of these notions, and used it to compare the three previous works. However, in each of these cases the adversary only receives leakage from decryption, and this leakage is modelled as a fixed, deterministic function, rather than a more general set of functions available to an adaptive side-channel attacker.

Leakage resilient constructions. Within the leakage resilient literature, there are several works towards providing leakage resilient encryption, but most of them have been in the bounded leakage model [24, 46]. In the bounded retrieval model, Bellare et al. [7] proved the security of a symmetric encryption scheme that provides authenticated encryption in the leak free case, and indistinguishability when leakage is involved. Pereira et al. [43] proposed what is, to our knowledge, the first and only leakage resilient encryption scheme in the simulatable leakage model. However, the construction requires a leak free component and in practice relies on the existence of efficient simulators of the leakage from (e.g.) AES, simulators that Longo et al. [33] demonstrate are unlikely to exist.

Following on from Pereira et al. [43], the recent work by Berti et al. [12] also attempts to construct leakage resilient misuse-resistent authenticated encryption, albeit from a very different direction. In some respects, our work is "top-down", setting a clear objective and evaluating what this demands of the underlying primitives, while theirs is "bottom-up", beginning with well understood primitives and asking what can be constructed. Motivated by this, the two papers adopt very different leakage models: we work in full generality, whereas different sections of Berti follow different leakage models. More generally, their work assumes a single (completely) leak free component, whereas ours allows any of the components to leak as long as the overall leakage is not too great. They hypothesis that (without many leak-free components) leakage resilient misuse resistant authenticated encryption is impossible, while we show that this can be achieved. Furthermore, their work does not consider associated data.

Another manner to ensure that the adversary cannot progressively leak the key material is to update the keys themselves (instead of their representation). Previous leakage resilient works in this direction include the MAC of Schipper [53], or the DH-ratcheting concept [16,44]. However, these tend to require that all parties to the communication hold modifiable state and remain perfectly in sync, a demand we are able to avoid.

Each of the models above severely restricts the information or computations that an adversary may be able to perform, thereby limiting their utility for modelling active side-channel attacks. The continuous leakage model mitigates these problems, which is why we focus on that when instantiating our AE scheme. To the best of our knowledge, ours is the first leakage resilient encryption scheme in the continuous leakage model. 
Our generic composition results allow us to combine leakage resilient components, for which we provide candidates built around a PRF secure against leakage. Currently there are two leakage resilient PRGs, due to Pietrzak (and Dziembowski) [19,45], from which it may be possible to build a leakage resilient stream cipher, although issues arise with restarting using the same key. Works of Dodis and Pietrzak [18], and Faust et al. [20] describe two PRFs secure under non-adaptive leakage: each requires that the leakage (functions) are fixed at the start of the game, while the latter also requires the inputs to be fixed. For a PRF to be used within a composition theorem, adaptive security is required. Finally, Martin et al. [36] provide a MAC which is secure against leakage on the tagging function only. We will use this as the basis of our instantiations, and extend it to achieve security against leakage on verification queries, resolving an open question from their work.

\section{Preliminaries}

General notation. For assignment of a value $U$ to the variable $T$ we will write $T \leftarrow U$, where $U$ may also be the outcome of some computation. If the variable is a set, we use the shorthand $S \leftarrow \cup U$ for $S \leftarrow S \cup\{U\}$. To assign a value drawn uniformly at random from some finite set $B$ to variable $A$, we write $A \leftarrow \$ B$. By convention, arrays and lists are initialised empty. We use $=$ for equality testing. We write $\mathbb{A} \rightarrow b$, to denote that adversary $\mathbb{A}$ outputs some value $b$. To define notions etc. we will write $X:=Y$ to say that $X$ is defined as some expression $Y$. The distinguished symbol $z$ denotes an invalid query. The symbol $\|$ denotes an unambiguous encoding, meaning if $Z \leftarrow X \| Y$ it must be possible given $Z$ to uniquely recover $X$ and $Y$, notated $X \| Y \leftarrow Z$, no matter what types $X, Y$ may take. The length $|A|$ is the length of $A$ when expressed as a string of elements of some underlying alphabet $\Sigma$ (usually $\Sigma=\{0,1\}$.

Whenever a function is described with a subscript, this will define the first parameter, meaning $f_{k}(\cdot, \cdot)=f(k, \cdot, \cdot)$. For consistency and clarity of notation, we refer to security definitions in capitals (e.g. IND-CPA) and typeset functions in calligraphic $(\mathcal{E})$, spaces in sans serif $(\mathrm{K})$, "secret" elements in lower case $(k)$, known elements in upper case $(M)$, and adversaries in blackboard bold $(\mathbb{A})$. When we introduce implementations, these will be denoted in bold $(\mathcal{E})$.

Adversarial advantages. We will define our security notions through indistinguishability games where an adversary is given access to one of two collections of oracles. The adversary $\mathbb{A}$ may make queries to these oracles, and eventually outputs a bit. Instead of writing the games in code, we adopt shorthand notation [2] so that the distinguishing advantage of $\mathbb{A}$ between two collections of $n$ oracles $\left(\mathcal{O}_{1}, \ldots, \mathcal{O}_{n}\right)$ and $\left(\mathcal{P}_{1}, \ldots, \mathcal{P}_{n}\right)$ is defined as

$$
\Delta_{\mathbb{A}}\left(\stackrel{\mathcal{O}}{1}_{\mathcal{P}_{1}}, \ldots, \mathcal{O}_{n}\right):=\left|\operatorname{Pr}\left[\mathbb{A}^{\mathcal{O}_{1}, \ldots, \mathcal{O}_{n}} \rightarrow 1\right]-\operatorname{Pr}\left[\mathbb{A}^{\mathcal{P}_{1}, \ldots, \mathcal{P}_{n}} \rightarrow 1\right]\right|,
$$

where the probabilities are taken over the randomness of the oracles, and key $k \leftarrow \$ \mathrm{~K}$ (note that multiple oracles will often use the same key). We may refer to the oracles by their numerical position: the $i^{\text {th }}$ oracle implements either $\mathcal{O}_{i}$ or $\mathcal{P}_{i}$ depending which collection the adversary is interacting with.

A scheme is considered secure with respect to a particular security goal if the relevant adversarial advantage is small for all adversaries running within reasonable resources. We do not draw judgement as to what "small" may mean, nor what constitutes "reasonable resources", since these depend heavily on context.

\subsection{Authenticated Encryption}

Core definitions. Early works to formalize symmetric encryption (cf. [27]) closely followed the precedent for public key encryption. Over the years understanding of what should be expected of symmetric encryption evolved considerably, both in terms of syntax and security. The basis for our work will be the widely accepted nonce-based model using indistinguishability from random bits for confidentiality [48-50]. After introducing this model, we will briefly refer back to an older, non-authenticated version of encryption as it is one of the building blocks later on. 


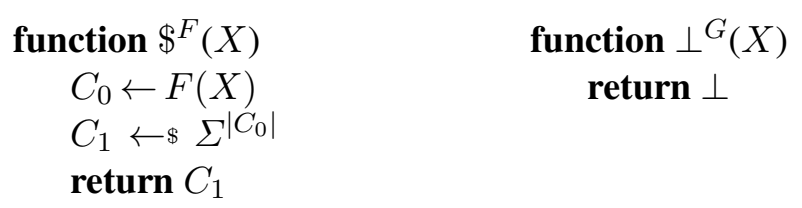

Fig. 1: The generic oracles $\$^{F}$ and $\perp^{G}$ idealise the output of $F$ as random elements of $\Sigma$, and of $G$ as always rejecting. They are used to define the reference world in our security definitions, for various choices of $(F, G)$, which will be omitted whenever clear. Usually $\Sigma=\{0,1\}$, with $\left|C_{0}\right|$ the length of $C_{0}$ as a bitstring.

Syntax. An authenticated encryption scheme consists of a pair of deterministic functions Enc and Dec, called encryption and decryption, respectively. Encryption Enc takes four inputs, resulting in a single ciphertext $C \in \mathrm{C}$. Besides the key $k \in \mathrm{K}$ and the message $M \in \mathrm{M}$, the inputs are some associated data $A \in \mathrm{A}$ that will be authenticated but not encrypted, and finally a nonce $N \in \mathrm{N}$ used to ensure that repeat encryptions will not result in repeat ciphertexts. Decryption Dec takes as input again the key, the nonce, and the associated data, in addition to the ciphertext. It outputs a purported message or an error message $\perp \notin \mathrm{M}$.

This syntax can be summarized as

$$
\begin{aligned}
& \text { Enc: } \mathrm{K} \times \mathrm{N} \times \mathrm{A} \times \mathrm{M} \rightarrow \mathrm{C} \\
& \text { Dec: } \mathrm{K} \times \mathrm{N} \times \mathrm{A} \times \mathrm{C} \rightarrow \mathrm{M} \cup\{\perp\} .
\end{aligned}
$$

In practice, the key space $K$, nonce space $N$, associated data $A$, message space $M$, and ciphertext space $C$ are generally bitstrings of various lengths. It is common to have $A=M=C=\{0,1\}^{*}$, and $K=$ $\mathrm{N}=\{0,1\}^{n}$ for some security parameter $n$. That said, our implementation in Appendix A instantiates the various spaces with more general groups (linked to pairings).

We require that an authenticated encryption scheme is both correct and tidy. These two properties are satisfied iff, for all $k, N, A, M, C$ in the appropriate spaces:

Correctness : $\operatorname{Dec}_{k}\left(N, A, \operatorname{Enc}_{k},(N, A, M)\right)=M$

Tidiness: $\quad$ if $\operatorname{Dec}_{k}(N, A, C) \neq \perp$ then $\operatorname{Enc}_{k}\left(N, A, \operatorname{Dec}_{k}(N, A, C)\right)=C$

Together, tidiness and correctness imply that decryption is wholly specified by the encryption routine.

Additionally, we require encryption to be length regular, which is satisfied if there exists some stretch function $\tau: \mathbb{N} \rightarrow \mathbb{N}$ such that for all inputs $\left|\operatorname{Enc}_{k}(N, A, M)\right|=|M|+\tau(|M|)$.

Security notions. Ever since Rogaway and Shrimpton's treatment of deterministic authenticated encryption, it is customary to capture both confidentiality and integrity requirements in a single game. Here the adversary gets oracle access either to the "real" world or to the "ideal" world and needs to distinguish between these two worlds. In the real world, oracle access consists of the encryption and decryption functionalities $\mathrm{Enc}_{k}$ and $\mathrm{Dec}_{k}$, using a randomly drawn and secret key $k$. In the ideal world, the encryption oracle is replaced with an oracle $\$$ that generates randomly drawn ciphertexts and the decryption oracle with an oracle $\perp$ that rejects all ciphertexts. Irrespective of which world the adversary is in, we will refer to the $\mathrm{Enc}_{k}$ vs. $\$$ oracle as the challenge encryption oracle or as the first oracle (based on the oracle ordering) and to the $\operatorname{Dec}_{k}$ vs. $\perp$ oracle as the challenge decryption (or second) oracle.

We will use a slightly different, but equivalent, formulation where an adversary additionally has access to the true encryption and decryption oracles in both worlds. Thus the adversary will have access to four oracles in each world: the challenge encryption oracle, the challenge decryption oracle, the true encryption oracle, and finally the true decryption oracle. Having these extra oracles will help us later on to add leakage, which will only ever be on the true oracles and never on one of the challenge oracles. One could even argue that the additional oracles provide a more representative and expressive framework: the honest oracles describe how an adversary may "learn" about a system, while the challenge ones allow them to "prove" they have done so (cf. a similar, more detailed argument for subtle authenticated encryption [4]).

As our reference point we will use the oracles defined in Figure 1, with all probabilities taken over randomness of the key and sampling within the oracle. 
Queries. Already in the leak-free setting, certain combinations of queries will easily distinguish the two worlds. To avoid these trivial wins, we will therefore prohibit certain queries—or in some cases simply assume adversaries refrain from making prohibited queries. For example, if an adversary can send a challenge encryption to decryption they can trivially win. As a general rule, we prohibit the same query being made to oracles which take the same inputs (such as the honest and challenge encryption oracles), and also prohibit performing the inverse of previous queries. For example, the ciphertext output from the challenge encryption oracle cannot be passed into the decryption oracle.

If an adversary has made a query $(N, A, M)$ to an encryption oracles (either challenge or true) receiving output $C$, then making the same query again to one of the encryption oracles or making the query $(N, A, C)$ to one of the decryption oracles (either challenge or true) the original and the new queries are deemed equivalent. For any query, we refer to the process of later making an equivalent query as forwarding the query, i.e. to make a second query whose inputs were inputs or outputs from the first query. A special case of forwarding a query is repeating the query, namely making the same query again to the same oracle. Forwarding queries from challenge to true oracles (or vice versa) or from challenge encryption to challenge decryption oracles (or vice versa) will lead to trivial wins unless oracle behaviour is adapted. Without loss of generality, we will restrict the adversary from making problematic queries instead.

Nonce selection requirements. Our security games will be agnostic over how the nonce is selected, with this property enforced by restricting the adversary. An adversary against an (authenticated) encryption scheme is called nonce respecting if whenever making a new query they do not use a nonce more than once to any oracle matching the syntax of $\mathrm{Enc}_{k}$ or $\mathcal{E}_{k}$. They are random-iv respecting, or simply iv respecting, if for any new query with these oracles their nonce $N$ (which we term an IV and will generally write as $I$ instead) is sampled uniformly from $\mathrm{N}$ immediately prior to querying the oracle (and thus not involved in the logic used to select other elements of the query). These requirements do not apply when interacting with oracles matching the syntax of $\operatorname{Dec}_{k}$ or $\mathcal{D}_{k}$. A scheme is called (nonce) misuse resistant if the adversary does not have to be nonce respecting, providing that the adversary does not make multiple queries using the same $(N, A, M)$ triple.

Definition 1 (nAE). Let Enc be an authenticated encryption scheme, $\mathbb{A}$ an adversary who does forward queries to or from his first or second oracle (and thus does not repeat first oracle queries). Then, the $n A E$ advantage of an adversary $\mathbb{A}$ against Enc is

$$
\left.\operatorname{Adv}_{\mathrm{Enc}}^{\mathrm{AE}}(\mathbb{A}):=\underset{\mathbb{A}}{\left(\mathrm{Enc}_{k}, \mathrm{Dec}_{k}, \mathrm{Enc}_{k}, \mathrm{Dec}_{k}\right.} \begin{array}{c}
\perp, \mathrm{Enc}_{k}, \operatorname{Dec}_{k}
\end{array}\right)
$$

Following our earlier convention, we will refer to a secure $n A E$ scheme (or simply $\mathrm{nAE}$ ) if this $\mathrm{nAE}$ advantage is small for all nonce-respecting adversaries running within reasonable resources, and mrAE if it is small for all adversaries running within reasonable resources that might repeat nonces.

Encryption. Authenticated encryption schemes are often constructed based on simpler encryption schemes. From a security perspective, the latter typically only provide a form of passive confidentiality and not integrity or authenticity. Consequently, these simpler encryption schemes do not take associated data as input, leading to the following syntax:

$$
\begin{aligned}
& \mathcal{E}: \mathrm{K} \times \mathrm{N} \times \mathrm{M} \rightarrow \mathrm{C} \\
& \mathcal{D}: \mathrm{K} \times \mathrm{N} \times \mathrm{C} \rightarrow \mathrm{M} \cup\{\perp\}
\end{aligned}
$$

where $\mathcal{E}$ is length-regular and $\mathcal{D}$ is uniquely determined by $\mathcal{E}$ to ensure both correctness and tidiness.

The standard confidentiality notion for an encryption scheme is indistinguishability under chosen plaintext attacks (Def. 2). For iv-based, or ivE, schemes, only iv-respecting adversaries are considered, whereas for nonce-based, or $\mathrm{nE}$, schemes only nonce-respecting adversaries are considered. 
Definition 2 (Indistinguishability under chosen plaintext attacks). Let $\mathcal{E}$ be an encryption scheme, and $\mathbb{A}$ an adversary who does not repeat first oracle queries or forward queries between his oracles. Then, the IND-CPA advantage of $\mathbb{A}$ against $\mathcal{E}$ is

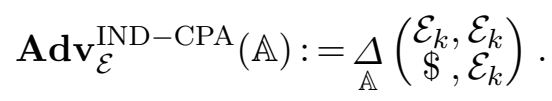

Building blocks: MACs and PRFs. A Message Authentication Code (MAC) consists of a pair of deterministic functions $(\mathcal{T}, \mathcal{V})$, named for Tag and Verify, having the syntax:

$$
\begin{aligned}
& \mathcal{T}: \mathrm{K} \times \mathrm{M} \rightarrow \mathrm{T} \\
& \mathcal{V}: \mathrm{K} \times \mathrm{M} \times \mathrm{T} \rightarrow\{\mathrm{T}, \perp\}
\end{aligned}
$$

where $T$ is called the tag space.

We restrict ourselves to deterministic MACs since our overall objective is to define a scheme that can be implemented in a deterministic way, and hence each component must be deterministic. In this setting, it is common to omit $\mathcal{V}$, since recomputating and comparing this with the candidate tag suffices (similar to how as encryption defines decryption for any tidy encryption schemes). We opt for the more descriptive setting and make $\mathcal{V}$ explicit, since later when we introduce leakage it will be important to differentiate between the tagging and verification implementations. Indeed, when leakage is available, simply recomputing the MAC is no longer sufficient to achieve security.

Definition 3 (Strong Existential Unforgeability under Chosen Message Attack). Let $(\mathcal{T}, \mathcal{V})$ be a (deterministic) MAC, and $\mathbb{A}$ an adversary who does not forward queries from his second oracle to the first. The Strong Existential Unforgeability under Chosen Message Attack (sEUF-CMA) advantage of $\mathbb{A}$ against $(\mathcal{T}, \mathcal{V})$ is the probability $\mathbb{A}$ can distinguish between interacting with $\mathcal{V}_{k}$ and $\perp$ when given access to $\mathcal{T}_{k}$ and $\mathcal{V}_{k}$. That is,

$$
\operatorname{Adv}_{(\mathcal{T}, \mathcal{V})}^{\mathrm{sEUF}-\mathrm{CMA}}(\mathbb{A}):=\underset{\mathbb{A}}{\Delta}\left(\begin{array}{l}
\mathcal{V}_{k}, \mathcal{T}_{k}, \mathcal{V}_{k} \\
\perp,
\end{array}, \mathcal{T}_{k}, \mathcal{V}_{k}\right)
$$

A keyed function $\mathcal{F}$ is called pseudorandom if for a randomly drawn secret key, it behaves as if it were a random function, formalized in Def. 4 below. It is easy to build a MAC from a pseudorandom function $\mathcal{F}$, simply by setting $\mathcal{T}_{k}=\mathcal{F}_{k}$ and $\mathcal{V}_{k}(M, T)=T \Longleftrightarrow \mathcal{F}_{k}(M)=T$. In that case a distinguishing adversary against the MAC in the sEUF-CMA game can be turned in an equally successful adversary against the PRF property of $\mathcal{F}$ (without incurring any significant overhead in the reduction).

Definition 4 (Pseudo Random Functions). Let $\mathcal{F}: \mathrm{K} \times \mathrm{M} \rightarrow \mathrm{T}$ be a function, and $\mathbb{A}$ an adversary who does not forward queries between their oracles or repeat first oracle queries. Then, the PRF advantage of $\mathbb{A}$ against $\mathcal{F}$ is

$$
\operatorname{Adv}_{\mathcal{F}}^{\mathrm{PRF}}(\mathbb{A}):=\underset{\mathbb{A}}{\Delta}\left(\begin{array}{c}
\mathcal{F}_{k}, \mathcal{F}_{k} \\
\$
\end{array}, \mathcal{F}_{k}\right)
$$

Generic composition for nAE. NRS [40] investigated how to construct an nAE scheme by composing two PRFs with an ivE scheme. The IV of the ivE scheme is derived from the nAE's inputs using the first PRF call; the optional second PRF call may be used to create an authentication tag. Different schemes emerge by changing which variables are provided to each of the components. NRS identify eight schemes, dubbed A1-A8, with strong security bounds. For a further four schemes (A9-A12) neither strong security bounds nor insecurity was established. Additionally, NRS investigated mechanisms for combining a PRF with an $\mathrm{nE}$ scheme. Three schemes (N1-N3) were found secure, with that of a fourth (N4) remaining unresolved.

Figure 2's middle panel shows the schemes A5 and A6. For these two schemes, as well as for N2 (on the left), the ciphertext is input to the second PRF, which means they classify as Encrypt-then-MAC (EtM). The schemes A4, A7-A12, as well as N3 and N4 only use a single PRF and release the IV as tag; for that reason we refer to them as MAC-then-Encrypt (MtE). Finally, the schemes A1-A3 and N1 use two PRFs that can be called in parallel, leading to their classification as Encrypt-and-MAC (E\&M). We refer to NRS for full descriptions and graphical illustrations of all schemes mentioned above. 


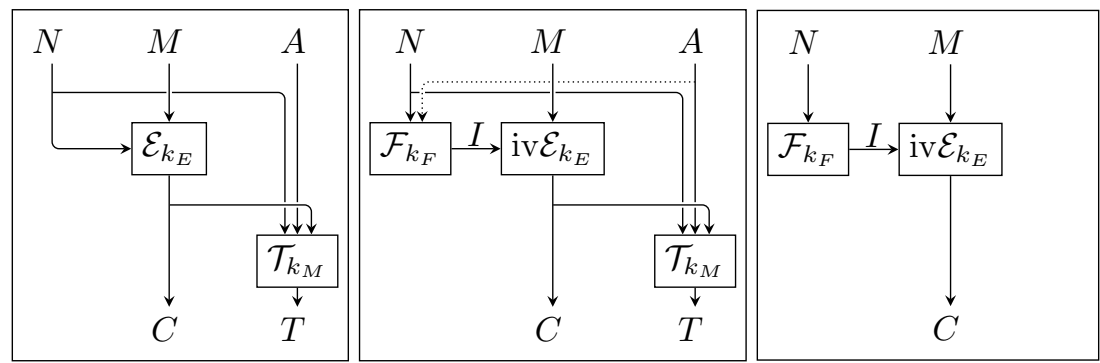

Fig. 2: Graphical representations of the encryption directions of generic composition mechanisms. On the left, N2 converts a nonce-based encryption algorithm $\mathcal{E}$ and MAC scheme $(\mathcal{T}, \mathcal{V})$ into an nAE scheme. On the right, iv2n converts an iv-based encryption scheme iv $\mathcal{E}$ and a $\mathrm{PRF}$ into a nonce-based encryption algorithm. Composing these yields $\mathrm{A} 5$, shown in the middle ignoring the dotted input, while $\mathrm{A} 6$ includes the dotted input. Overall decryption of $\mathrm{A} 5, \mathrm{~A} 6$, and $\mathrm{N} 2$ will recompute and verify the tag first, only proceeding with further decryption of $C$ if this verification is successful.

\section{Security Notions Involving Leakage}

Authenticated encryption, as defined above, is deterministic. In a leakage-free setting, this provides a stronger notion than the older probabilistic notion of encryption (as implicitly still used for ivE). When introducing leakage, deterministic schemes are problematic both from a practical and a theoretical perspective.

On the one hand, a practical side-channel attack such as differential power analysis can effectively recover keys from unprotected blockciphers and their AE modes with near certainty. Randomized masking based on secret sharing is one of the main countermeasures against these attacks.

On the other hand, theoretical leakage is often modelled as a function on the inputs of the computation, which will include the key. If with each invocation of the scheme an adversary can let the scheme leak a different key bit of its choice, the full key is easily recovered. To prevent such devastating yet simple leakage, a typical design strategy is to split the key in two shares and update the shares on-the-fly using fresh randomness, mimicking the practical approach.

\subsection{Implementations Versus Functions}

In both the practical and the theoretical approaches mentioned above, a deterministic scheme is implemented in a randomized fashion in order to provide resistance against leakage. Therefore, when arguing about leakage, we will need to make a distinction between the scheme (a collection of deterministic functions) and its probabilistic implementation.

For our definition of the implementations of a function we take our cue from the secret-sharing approach, where a redundant representation of the key is used and this representation is rerandomized as part of the implementation. To enable this rerandomization, we provide the implementation of a function with explicit randomness in Definition 5 below, where we use a bold font to denote either the implementation of a function or the representation of a key used by the implementation.

Definition 5 (Implementation of $f$ ). An implementation of a function $f: \mathrm{K} \times \mathrm{X} \rightarrow \mathrm{Y}$ is a deterministic function $\boldsymbol{f}: \mathrm{K} \times \mathrm{X} \times \mathrm{R} \rightarrow \mathrm{K} \times \mathrm{Y}$ along with a probabilistic key initialisation function $\iota: \mathrm{K} \rightarrow \mathbf{K}$ such that $\iota(k)=\iota(l) \Rightarrow k=l$. We define the inverse of $\iota$ as the function $\iota^{-1}: \mathbf{K} \rightarrow \mathrm{K} \cup\{\perp\}$ such that $\iota^{-1}(\boldsymbol{k})=k$ if $\iota(k)$ could have resulted in $\boldsymbol{k}$, and $\perp$ if no such $k$ exists.

The implementation is correct iff for all $k \in \mathrm{K}, X \in \mathrm{X}$, and $r \in \mathrm{R}$, setting $\boldsymbol{k} \leftarrow \iota(k)$ and $\left(\boldsymbol{k}^{\prime}, Y\right) \leftarrow \boldsymbol{f}(\boldsymbol{k}, X ; r)$ guarantees both $Y=f(k, X)$ and $\iota^{-1}\left(\boldsymbol{k}^{\prime}\right)=k$.

The initial representation of the key is generated using the function $\iota$, which maps a key $k \in \mathrm{K}$ to a suitable representation $\boldsymbol{k} \in \mathbf{K}$ for the implementation. We assume that $\iota$ is performed only once, and in a leak-free manner, during setup (straight after key generation). Moreover, its inverse $\iota^{-1}$ induces an equivalence relation on the space $\mathbf{K}$; in other words, the implementation keys $k$ can be thought of as alternative representations of the key. During evaluation of $\boldsymbol{f}$ the auxiliary input $r \in \mathrm{R}$ is used to refresh the representation; typically this requires a good randomness source to draw $r$ from. 
Discussion. Correctness implies that an implementation is identical to the original function when restricted to the second output and that the new key representation $\boldsymbol{k}^{\prime}$ is equivalent to the initial one $\boldsymbol{k}$. We make no demands of $\boldsymbol{k}$ or $\boldsymbol{k}^{\prime}$ beyond these, so it is permissible to set $\boldsymbol{k}=\boldsymbol{k}^{\prime}=k$ and thus recover the traditional syntax. Our security definitions will be such that for correct schemes and assuming "trivial" leakage, the corresponding leak-free security notions from the preceding section will emerge.

Definition 5 can be linked to practice in a straightforward manner. Recall that practical implementations of blockciphers often use masking based on secret sharing schemes. In this case, the implementation of the blockcipher describes how to evaluate the blockcipher based on the shares of the key as well as how the sharing is refreshed using external randomness $r$ (which need not be leak-free). Furthermore, $\iota$ is exactly the function that creates the initial secret sharing of the key.

Syntactically the implementation $\boldsymbol{f}$ may appear stateful: after all they take in some $\boldsymbol{k}$ and output an updated $\boldsymbol{k}^{\prime}$ for the next invocation. However, since the implementation is of a stateless function $f$, there is no need to synchronize state between communication parties. Instead, each party can use its own, independent representation of the key.

Implementation of an nAE Scheme. For concreteness, we now explicitly define the implementation of an nAE scheme. We assume that Enc and Dec syntactically use the same representations $\mathbf{K}$ (and key initialisation function $\iota$ ), which we later use for expressing our security notions.

By correctness of the implementation, one can see that the ciphertext output by Enc (resp. message by Dec) will always be independent of the randomness $r$, since they are equal to the corresponding output of Enc (resp. Dec). Definitions for the implementations of other security primitives are written accordingly.

Definition 6 (AE Implementation). Let (Enc, Dec) be an authenticated encryption scheme. An AE implementation is a pair of deterministic functions

$$
\begin{aligned}
& \text { Enc: } \mathbf{K} \times \mathbf{N} \times \mathbf{A} \times \mathrm{M} \times \mathrm{R} \rightarrow \mathbf{K} \times \mathrm{C} \\
& \text { Dec: } \mathbf{K} \times \mathrm{N} \times \mathrm{A} \times \mathrm{C} \times \mathrm{R} \rightarrow \mathbf{K} \times(\mathrm{M} \cup\{\perp\})
\end{aligned}
$$

along with $\iota: \mathrm{K} \rightarrow \mathbf{K}$ satisfying $\iota(k)=\iota(l) \Rightarrow k=l$ and $\iota^{-1}: \mathbf{K} \rightarrow \mathrm{K} \cup\{\perp\}$ such that $\iota^{-1}(\boldsymbol{k})=k$ if $\iota(k)$ could have resulted in $\boldsymbol{k}$, and $\perp$ if no such $k$ exists. The implementation is correct iff for any $k, N, A, M, C, r$ from the appropriate spaces and $\boldsymbol{k} \leftarrow \$ \iota(k)$, setting

$$
\left(\boldsymbol{k}^{\prime}, C^{\prime}\right) \leftarrow \operatorname{Enc}(\boldsymbol{k}, N, A, M ; r) \text { and }\left(\boldsymbol{k}^{\prime \prime}, M^{\prime}\right) \leftarrow \operatorname{Dec}(\boldsymbol{k}, N, A, C ; r),
$$

$\left(\boldsymbol{k}^{\prime}, C^{\prime}\right) \leftarrow \operatorname{Enc}(\boldsymbol{k}, N, A, M ; r)$ and $\left(\boldsymbol{k}^{\prime \prime}, M^{\prime}\right) \leftarrow \mathbf{D e c}(\boldsymbol{k}, N, A, C ; r)$, the following properties hold:

$$
\begin{aligned}
& k=\iota^{-1}(\boldsymbol{k})=\iota^{-1}\left(\boldsymbol{k}^{\prime}\right)=\iota^{-1}\left(\boldsymbol{k}^{\prime \prime}\right) \\
& C^{\prime}=\operatorname{Enc}_{k}(N, A, M) \text { and } M^{\prime}=\operatorname{Dec}_{k}(N, A, C) .
\end{aligned}
$$

\subsection{What Constitutes Leakage}

Following Micali and Reyzin's approach, we will model leakage by allowing an adversary to specify a leakage function in conjunction with an oracle query. The input signature of the leakage function matches that of the implementation $f$ it relates to, allowing it to wholly simulate the implementation. A leakage set is a collection of leakage functions for an implementation.

Definition 7 (Leakage Functions). A leakage function of an implementation $f: \mathrm{K} \times \mathrm{X} \times \mathrm{R} \rightarrow \mathrm{K} \times \mathrm{Y}$ is a function $L: \mathrm{K} \times \mathrm{X} \times \mathrm{R} \rightarrow \mathrm{L}$ for some output leakage space $\mathrm{L}$. A leakage set of an implementation $f$ is a set of leakage functions.

The choice of leakage set should contain all plausible (functions of) inputs to the implementation that an adversary can compute, and may be probabilistic. This might include functions of any intermediate 

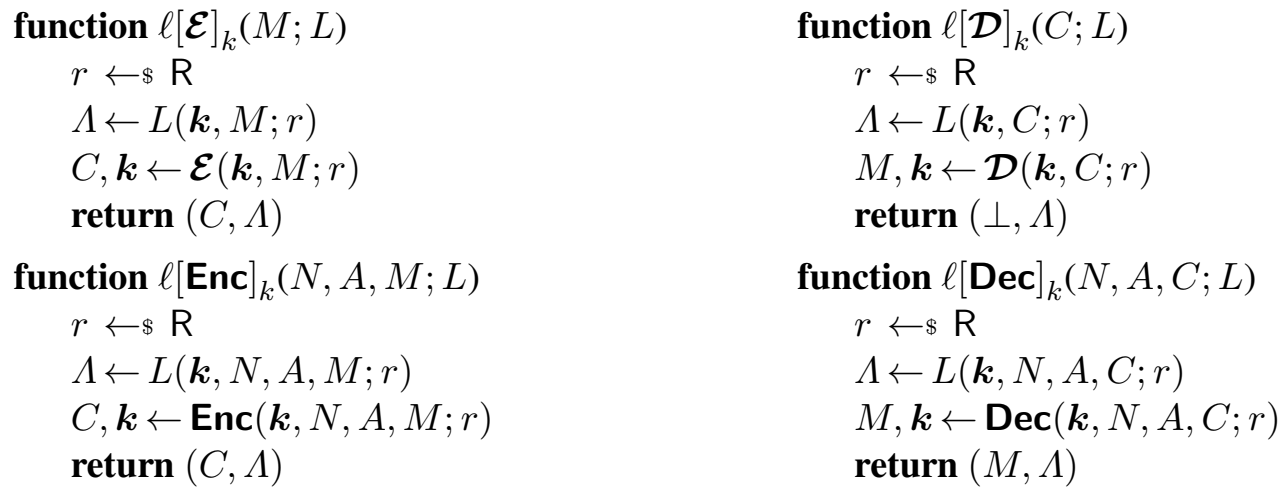

Fig. 3: Honest leakage oracles an adversary may use to help them distinguish. All inputs are taken from the appropriate spaces, with leakage functions chosen from the relevant leakage set. For $\mathcal{L}_{\mathrm{E}}$-IND-CPLA, the adversary has access to $\ell[\mathcal{E}]_{k}$, and for the augmented notion $\left(\mathcal{L}_{\mathrm{E}}, \mathcal{L}_{\mathrm{D}}\right)-$ IND-aCPLA they are also given very limited access to $\ell[\mathcal{D}]_{k}$. LAE security, $\left(\mathcal{L}_{\text {Enc }}, \mathcal{L}_{\text {Dec }}\right)$-LAE provides access to $\left(\ell[\text { Enc }]_{k}, \ell[\text { Dec }]_{k}\right)$.

variables, since these are computable from the inputs simply by simulating the construction. Broadly speaking, the larger the leakage set the more powerful the adversary is likely to be. The leakage set $\emptyset$ allows us to model the leak-free case. Technically we define it to be the set containing just the null function, meaning the adversary can always select a leakage function, thus maintaining the correct syntax for our security games.

\subsection{Security Notions Incorporating Leakage}

We are now in a position to define the security of an implementation in the presence of leakage. We do so by reframing the classical notions given to work on the implementation of a function, and by extending the notions such that the honest oracles are allowed to leak. The adversary wins the game if they can distinguish whether their leak-free challenge oracles implement the scheme honestly or are idealised. We differentiate our notions from the classic variant by prefixing an "L", for leakage.

In the classical setting, each oracle simply evaluates the appropriate function with the game's secret key. For an implementation, a similar, but slightly more complicated, approach is required. The oracle must draw randomness, and provide this to the implementation to update the key representation. This same randomness, along with all other inputs, must be provided to the leakage function. The new representation must then be stored, and the two outputs returned to the adversary. For any implementation $\boldsymbol{f}$, the corresponding leakage oracle is denoted $\ell[\boldsymbol{f}]_{k}$, when initialised with representation $\boldsymbol{k}=\iota(k)$. Code-based descriptions for certain leaky implementations related to authenticated encryption are given in Figure 3. If an adversary has access to multiple oracles based on the same key, say $\mathrm{Enc}_{k}$ and $\mathrm{Dec}_{k}$, then we will assume that their respective implementation oracles (so $\ell[\mathbf{E n c}]_{k}$ and $\ell[\mathbf{D e c}]_{k}$ ) will operate on the same representation $\boldsymbol{k}$, which hence will be initialized only once. Such a shared representation corresponds to a setting where both Enc and Dec are implemented on the same device. Needless to say, our security definitions below can be strengthened by allowing an adversary to interact with multiple implementations each using their own representation of the same key.

As in the leakage free definitions, security is taken over the randomness of the initial keys, and of the oracles. Notice that this choice includes the sampling from R. We assume the adversary only ever makes queries for which his inputs are selected from the appropriate spaces. For leakage, this means some leakage set that will be specified in the security notion.

For the purposes of defining forwarding of queries, we will ignore the additional input associated to the leakage. For instance, after a query $(N, A, M)$ to the challenge encryption oracle, the query $(N, A, M, L)$ to the true encryption oracle will be considered equivalent—and would constitute forwarding-irrespective of $L$.

Definition 8 (AE security against leakage). Let (Enc, Dec) be an implementation of an authenticated encryption scheme Enc, Dec, and $\mathbb{A}$ an adversary who does not forward queries to or from his first or second oracles (and thus does not repeat such queries). Then, the $\left(\mathcal{L}_{\mathrm{Enc}}, \mathcal{L}_{\text {Dec }}\right)-\mathrm{LAE}$ advantage of an 
adversary $\mathbb{A}$ against $($ Enc, Dec $)$ under leakage $\left(\mathcal{L}_{\mathrm{Enc}}, \mathcal{L}_{\mathrm{Dec}}\right)$ is

$$
\mathbf{A d v}_{\mathrm{Enc}, \operatorname{Dec} ; \mathcal{L}_{\mathrm{Enc}}, \mathcal{L}_{\mathrm{Dec}}^{\mathrm{LAE}}}(\mathbb{A}):=\underset{\mathbb{A}}{\Delta}\left(\begin{array}{c}
\mathrm{Enc}_{k}, \mathrm{Dec}_{k}, \ell[\mathbf{E n c}]_{k}, \ell[\mathbf{D e c}]_{k} \\
\$, \perp, \ell[\mathbf{E n c}]_{k}, \ell[\mathbf{D e c}]_{k}
\end{array}\right)
$$

Definition 9 (Encryption security against leakage). Let $\mathcal{E}$ be an implementation of an encryption scheme $\mathcal{E}$, and $\mathbb{A}$ an adversary who never forwards queries to or from his first oracle (and thus does not repeat first oracle queries). The $\mathcal{L}_{\mathrm{E}-I N D-C P L A}$ advantage (named for chosen-plaintext-with-leakageattack) of $\mathbb{A}$ against $\mathcal{E}$ is

$$
\operatorname{Adv}_{\mathcal{E} ; \mathcal{L}_{\mathrm{E}}}^{\mathrm{IND}-\mathrm{CPLA}}(\mathbb{A}):=\underset{\mathbb{A}}{\Delta}\left(\begin{array}{c}
\mathcal{E}_{k}, \ell[\mathcal{E}]_{k} \\
\$, \ell[\mathcal{E}]_{k}
\end{array}\right)
$$

We next provide an additional encryption notion, IND-aCPLA, that will be required for our composition results later. It describes a modified version of the IND-CPLA game in which the adversary is also allowed leakage from the decryption implementation $\ell[\mathcal{D}]_{k}$ (see Figure 3), but only on ciphertexts they have previously received from $\ell[\mathcal{E}]_{k}$. At first glance, this appears to be more similar to an INDCCA style notion, but we emphasise this is not the case since the possible decryption queries are heavily restricted. Thus it should be thought of as IND-CPA under the most general form of leakage. Indeed, when the leakage sets are empty, the resulting security notion is equivalent to IND-CPA.

Definition 10 (Augmented CPLA security). Let $(\mathcal{E}, \mathcal{D})$ be an implementation of an encryption scheme, $\mathbb{A}$ an adversary who does not forward queries to or from his first oracle, and only makes queries to their third oracle that were forwarded from the second. Then the $\left(\mathcal{L}_{\mathrm{E}}, \mathcal{L}_{\mathrm{D}}\right)$-IND-aCPLA advantage of $\mathbb{A}$ against $\mathcal{E}$ is

$$
\operatorname{Adv}_{\mathcal{E}, \mathcal{D} ; \mathcal{L}_{\mathrm{E}}, \mathcal{L}_{\mathrm{D}}}^{\mathrm{IND}}(\mathbb{A}):=\underset{\mathbb{A}}{\Delta}\left(\begin{array}{c}
\mathcal{E}_{k}, \ell[\mathcal{E}]_{k}, \ell[\mathcal{D}]_{k} \\
\$, \ell[\mathcal{E}]_{k}, \ell[\mathcal{D}]_{k}
\end{array}\right)
$$

The IND-aCPLA notion is required for the general composition, where the goal is to construct an LAE scheme from an ivLE scheme (and other components). However, for decryption of the LAE scheme to leak (as we want the leakage to be as powerful as possible), the decryption of ivLE scheme would have to leak. The IND-CPLA security notion does not capture this. Consider an IND-CPA scheme where encryption does not leak, but the leakage from decrypting the zero string returns the key. Clearly the scheme is also IND-CPLA but will trivially break when the adversary is given decryption leakage. The IND-aCPLA notion is trying to capture that decryption "does not leak too much information", so that limited decryption queries made by the LAE scheme will be able to leak.

Against many natural choices of leakage sets, $\left(\mathcal{L}_{\mathrm{E}}, \mathcal{L}_{\mathrm{D}}\right)$-IND-aCPLA and $\mathcal{L}_{\mathrm{E}}$-IND-CPLA are equivalent, since the encryption oracle often suffices to simulate any leakage from decryption. In the nonceabusing setting (where the adversary is free to select nonces however they wish) there is an obvious mechanism for proving the equivalence, using repeat encryption queries to simulate leaking decryption queries, but even this requires rather strong assumptions on the leakage sets.

In the nonce respecting or iv respecting scenarios such a general reduction is not possible, because there is no way to allow the adversary to use the same nonce multiple times, something a decryption oracle would allow. If the leakage is independent of the nonce (for example) similar results can be recovered, but these are much more restrictive scenarios. It is an interesting open problem to describe sets $\mathcal{L}_{\mathrm{ED}}$ that are in some sense "minimal" for various pairs of leakage sets $\left(\mathcal{L}_{\mathrm{E}}, \mathcal{L}_{\mathrm{D}}\right)$ taken from some general function classes.

LMAC and LPRF. Here we give the PRF and MAC notions a similar treatment to the encryption definitions by enhancing the standard definitions (Appendix 2.1) to incorporate leakage.

The LPRF definition below strengthens earlier definitions by Dodis and Pietrzak [18], and by Faust et al. [20]: in our definition both the leakage functions and the inputs can be chosen adaptively based on outputs already seen by the adversary. 

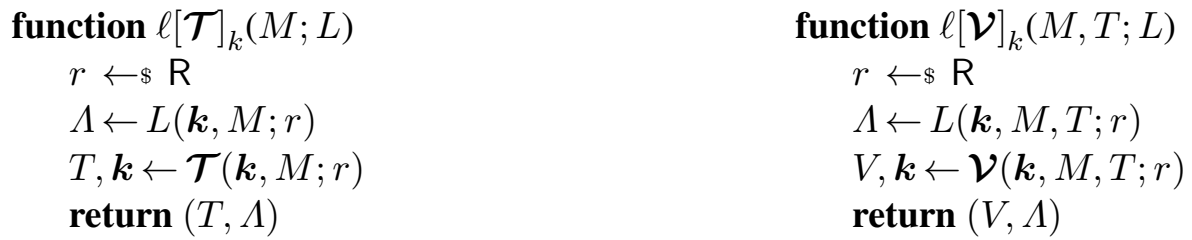

Fig. 4: Honest leakage oracles an adversary may use to help them distinguish. All inputs are taken from the appropriate spaces, with leakage functions chosen from the relevant leakage set. $\left(\mathcal{L}_{\mathrm{T}}, \mathcal{L}_{\mathrm{V}}\right)$-LMAC security gives access to $\left(\ell[\mathcal{T}]_{k}, \ell[\mathcal{V}]_{k}\right)$. Since PRFs and the tagging function of a MAC have the same syntax, the LPRF game provides access to $\ell[\mathcal{F}]_{k}$, which is identical to $\ell[\mathcal{T}]_{k}$.

Definition 11 (PRF security against leakage). Let $\mathcal{F}$ be an implementation of a function $\mathcal{F}$, and $\mathbb{A}$ an adversary who never forwards or repeats queries. Then the $\mathcal{L}_{\mathrm{F}}-P R L F$ advantage of $\mathbb{A}$ against $\mathcal{F}$ under leakage $\mathcal{L}_{\mathrm{F}}$ is

$$
\operatorname{Adv}_{\mathcal{F} ; \mathcal{L}_{\mathrm{F}}}^{\mathrm{PRLF}}(\mathbb{A}):=\Delta \underset{\mathbb{A}}{\Delta}\left(\begin{array}{c}
\mathcal{F}_{k}, \ell[\mathcal{F}]_{k} \\
\$, \ell[\mathcal{F}]_{k}
\end{array}\right)
$$

Our notion of strong existential unforgeability under chosen message with leakage (below) strengthens both the classical definition, and the leakage definition of Martin et al. [36] (they only allow tagging to leak; setting $\mathcal{L}_{\mathrm{V}}=\emptyset$ recovers their definition). Allowing meaningful leakage on $\mathcal{T}$ hampers direct use of a secure LPRF as a MAC as typically during verification the "correct" tag would be recomputed as output of the PRF and could consequently be leaked upon (effectively yielding a surreptitious tagging algorithm).

Definition 12 (MAC security against leakage). Let $(\mathcal{T}, \mathcal{V})$ be an implementation of a $M A C(\mathcal{T}, \mathcal{V})$, and $\mathbb{A}$ an adversary who does not forward queries from his second oracle to the first. Then the $\left(\mathcal{L}_{\mathrm{T}}, \mathcal{L}_{\mathrm{V}}\right)$ sEUF-CMLA advantage of $\mathbb{A}$ against $(\mathcal{T}, \mathcal{V})$ under leakage $\left(\mathcal{L}_{\mathrm{T}}, \mathcal{L}_{\mathrm{V}}\right)$ is

$$
\operatorname{Adv}_{\mathcal{T}, \mathcal{V} ; \mathcal{L}_{\mathrm{T}}, \mathcal{L}_{V}}^{\mathrm{sEUF}}(\mathbb{A}):=\underset{\mathbb{A}}{\Delta}\left(\begin{array}{l}
\mathcal{V}_{k}, \ell[\mathcal{T}]_{k}, \ell[\mathcal{V}]_{k} \\
\perp, \ell[\mathcal{T}]_{k}, \ell[\mathcal{V}]_{k}
\end{array}\right)
$$

Note that we cast unforgeability as a distinguishing game, rather than as a more usual computational game ("adversary must forge a tag"), but it is straightforward to show equivalence (even in the face of leakage).

\section{Applying LAE to Attacks in Theory and Practice}

A security framework is not much use if it does not highlight the difference between schemes for which strong security results are known, and those against which efficient attacks exist. In this section we discuss the types of leakage normally considered within the literature. We show how previous leakage models can be captured by our leakage set style notion. In the literature there is focus on two types of leakage; protocol leakage (by the AE literature) and side channel leakage (by the leakage resilient literature). We believe that these two notions are highly related and thus we discuss how to capture both. For example, termination of an algorithm at different points (distinguishable decryption failures) is normally detected by a side-channel; timing can be used to capture this if the failures terminate the algorithm at different points in time and power can be used to detect if conditional branches were taken.

After first describing generic methods to recast existing leakage resilience work within our general framework, we consider a concrete example of this, describing an existing attack within our setting.

\subsection{Theoretical Leakage Models}

We observe that our model is in many ways the most general possible, and that many previous leakage notions can be captured as version of the $\left(\mathcal{L}_{\mathrm{E}}, \mathcal{L}_{\mathrm{D}}\right)$-LAE security game for suitable choice of leakage sets $\left(\mathcal{L}_{\mathrm{E}}, \mathcal{L}_{\mathrm{D}}\right)$. Reassuringly, by setting $\left(\mathcal{L}_{\mathrm{E}}, \mathcal{L}_{\mathrm{D}}\right)=(\emptyset, \emptyset)$ we recover the traditional leakage-free security notions, with $(\emptyset, \emptyset)$-nLAE equivalent to nAE, and both $\emptyset$-IND-CPLA and $(\emptyset, \emptyset)$-IND-aCPLA equivalent to IND-CPA, meaning a secure $\mathrm{nE}$ scheme is $\emptyset$-nLE secure. 
The deterministic decryption leakage notions from the AE literature can be recovered by choosing the appropriate leakage set. The SAE framework generalises both the RUP model, and (nonce-based analogues of) the Distinguishable Decryption Failure notions of Boldyreva et al. $[2,4,13]$. The security notions are parametrised by a deterministic decryption leakage function $\Lambda$, corresponding to security under the leakage sets $\left(\mathcal{L}_{\mathrm{E}}, \mathcal{L}_{\mathrm{D}}\right)=(\emptyset,\{\Lambda\})$. Thus the strongest notions available in these settings are equivalent to $(\emptyset,\{\Lambda\})$-LAE. Several of their weaker notions translate to the corresponding weakening of this, including authenticity under deterministic leakage, (known variously as CTI-sCPA, INT-RUP or an extended form of INT-CTXT), which translates to a variant of $(\emptyset,\{\Lambda\})$-LAE in which the adversary cannot query the encryption challenge oracle (and thus does not interact with either $\mathcal{E}_{k}$ or $\$$ ).

In the simulatable leakage model (e.g. [56]), the adversary receives leakage in addition to their query, but is restricted to leakage functions that can be simulated without the key. The simulatable model considered by Standaert et al. (for example) can be captured by our model by having set of leakage functions contain the single function which provides the power trace to the adversary. The auxiliary input model [17] gives the adversary the output of a hard to invert function applied to the key, alongside the normal security notion interactions. The only computation leaks model [39] (discussed in more detail in Section 5.1) restricts the adversary to leakage functions that can be locally computed: any step of the algorithm can only leak on variables being used at that point. In the following sections we show how this leakage set can be defined for our given constructions.

In the probing model [26] the adversary can gain access to the values of $t$ of the internal wires from the computation. A scheme is secure if an adversary with the knowledge of $t$ internal wires can do no better than if they had access to the function in a black box manner. If there are $n$ internal wires, this leakage can be captured by our set notation by constructing a set with $n$ choose $t$ leakage functions, each giving the complete value of the relevant wires.

Our leakage sets incorporate the bounded leakage model (e.g. [24, 28, 32]) by restricting the set of allowable adversaries to those who only make sufficiently few queries to the leakage oracles.

One mechanism that need not rely on randomness is to instead use a leak-free component [58]. Although instantiating such components in practice is between hard and impossible [35], our framework nonetheless supports it (by suitable choice of leakage set).

Another idea to provide security is frequent rekeying. However, such a solution relies on synchronized states between encryption and decryption which can be difficult to maintain, thereby restricting applicability of this approach. However, in specific contexts such as secure channels, synchronization might not be too onerous.

\subsection{Casting an Existing Attack against GCM into Our Framework}

As a concrete example of how to set an existing attack within the framework, consider the attack by Belaïd, Fougue and Gérard [5] against the well-known AES-GCM [38] AEAD mode. The authors observe that that the least significant bit of a vector's Hamming weight is simply a linear combination of its bits. Therefore, if the Hamming weight (or an approximation of it) is available, then (some approximation) of this linear combination can be deduced. Based on this, they construct an attack on the authentication key, using leakage from the first polynomial multiplication.

The GCM encryption routine and the relevant leakage oracle are given in Figure 5. Note that as the implementation under attack was not randomised, the oracle's randomness $r$ is not used. To apply the attack, we require that $L(k, N, A, M ; r)=\operatorname{HW}\left(A_{1} \bullet \mathrm{E}_{k}\left(0^{128}\right)\right) \oplus \varepsilon$ is an element of the encryption leakage set $\mathcal{L}_{\mathrm{E}}$, or that the corresponding function $L(k, N, A, C ; r)$ is in $\mathcal{L}_{\mathrm{D}}$. Within GCM, the value $H=\mathrm{E}_{k}\left(0^{128}\right)$ is the authentication key, so this leakage function calculates its Hamming weight (HW) after multiplication by a known quantity $A_{1}$, before adding some Gaussian noise $\varepsilon$ to better describe experimental error.

The adversary makes a series of queries to their honest oracles to acquire this leakage, and then collects the least significant bit of the each leakage query, along with the corresponding value $A_{1}$, to form a system of (noisy) linear equations. In the most naive case, if it is possible to guess where the noise causes errors in the linear equations, they can be solved in the standard manner to find $H=\mathrm{E}_{k}\left(0^{128}\right)$, 


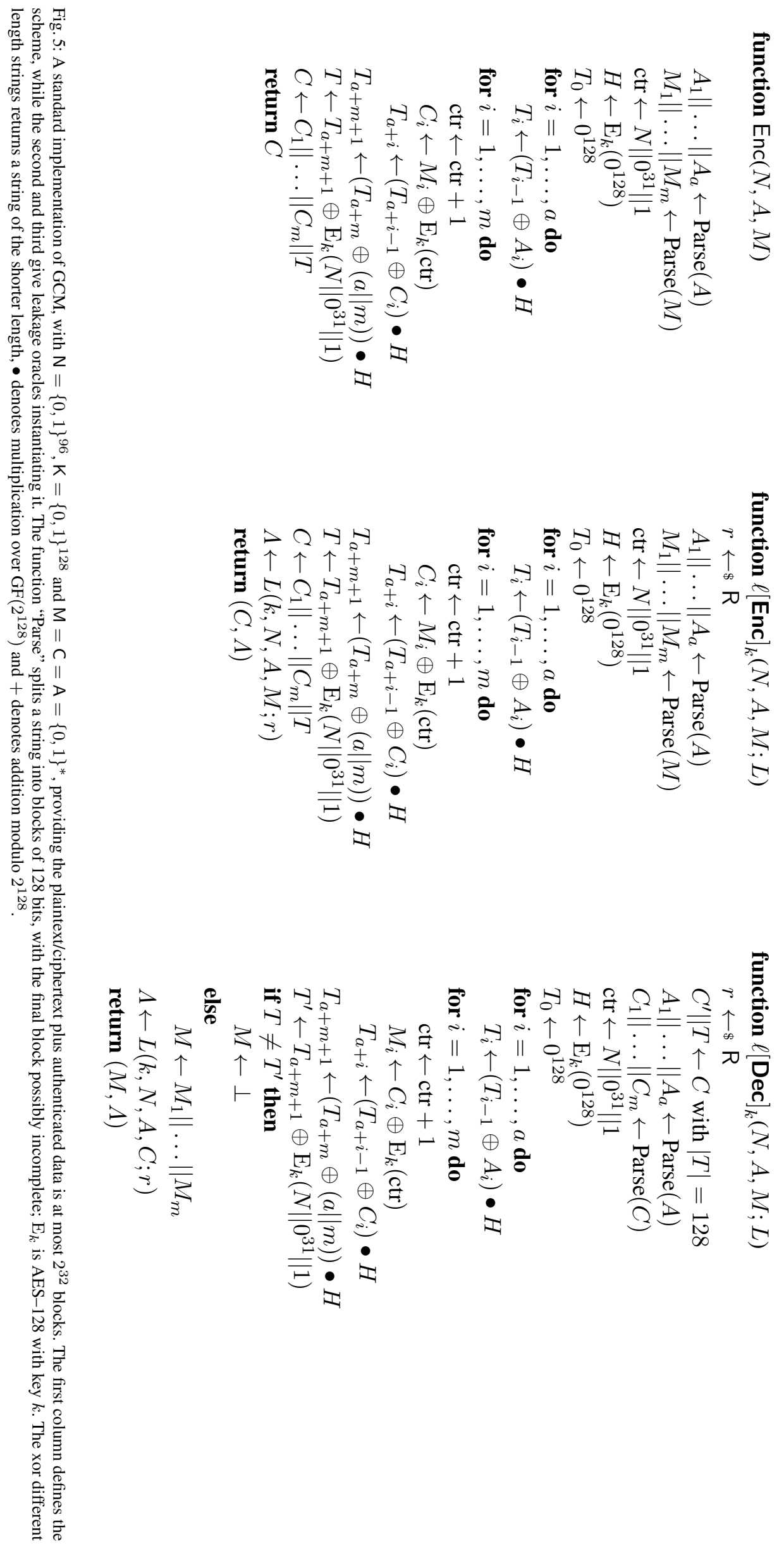


which completely breaks the security of the scheme. For 6 errors this requires approximately $2^{32}$ effort. Other elements of the original work expand upon this, providing more involved techniques for reducing the amount of work required to solve this noisy system.

Overall then, this attack demonstrates that the implementation of AES-GCM described in Figure 5 is neither $(\emptyset,\{L\})$-nLAE nor $(\{L\}, \emptyset)$-nLAE.

\section{Generic Composition for LAE}

\subsection{Modelling Composed Leakage}

Our challenge is to establish to what extent the NRS schemes remain secure when taking leakage into account. Ideally, we would like to claim that if both the ivE and the PRFs are secure in the presence of leakage, then so will the composed nAE be. To make such a statement precise, the leakage classes involved need to be specified. We opt for an approach where the leakage classes for the components are given (and can be arbitrary) and then derive a leakage class for the resulting nAE for which we can prove security.

Encryption leakage. In a nutshell, we define the leakage of the composition as the composition of the leakage. As an example, consider an implementation of A5 (Figure 2). When encrypting, the leakage may come from any of the components: the PRF $\mathcal{F}$ may leak some information $L_{F}\left(\boldsymbol{k}_{F}, N ; r_{F}\right)$; the IVencryption routine iv $\mathcal{E}$ might leak some information $L_{E}\left(\boldsymbol{k}_{E}, I, M ; r_{E}\right)$; the Tag function $\mathcal{T}$ may leak some information $L_{T}\left(\boldsymbol{k}_{M}, N, A, C_{e} ; r_{M}\right)$. To ease notation, we will use the shorthand $L_{F}(\star), L_{E}(\star)$, and $L_{T}(\star)$ respectively for these leakages. In that case, we say that the leakage on the authenticated encryption operation as a whole consists of the triple $\left(L_{F}(\star), L_{E}(\star), L_{T}(\star)\right)$. Under the hood, this implies some parsing and forwarding of the AE's key $\left(\boldsymbol{k}_{F}, \boldsymbol{k}_{E}, \boldsymbol{k}_{M}\right)$, randomness $\left(r_{F}, r_{E}, r_{M}\right)$ and inputs $N, A, M$, including the calculated values $I$ and $C_{e}$, to the component leakage functions $L_{F}, L_{E}$, and $L_{T}$.

Expanding the above to classes of functions is as follows. Let $\mathcal{L}_{\mathrm{F}}, \mathcal{L}_{\mathrm{E}}$, and $\mathcal{L}_{\mathrm{T}}$ be the respective leakage classes for $\mathcal{F}$, ive $\mathcal{E}$, and $\mathcal{T}$. Then the leakage class $\mathcal{L}_{\text {Enc }}$ for the resulting authenticated encryption scheme is defined as $\left\{\left(L_{F}, L_{E}, L_{T}\right) \mid L_{F} \in \mathcal{L}_{\mathrm{F}}, L_{E} \in \mathcal{L}_{\mathrm{E}}, L_{T} \in \mathcal{L}_{\mathrm{T}}\right\}$. Since an adversary has to select a leakage function in $\mathcal{L}_{\mathrm{Enc}}$ the moment it queries the encryption oracle, it will not be possible to adaptively select for instance the leakage function $L_{T}$ based on the leakage received from $L_{E}$ of that encryption query.

Decryption leakage. In order to describe leakage from decryption, we take a closer look at the role of the two PRFs in the generic constructions. The first one, $\mathcal{F}$, computes the initial vector which is needed both for encryption and decryption. This makes it inevitable that during decryption $\mathcal{F}$ is again computed as a PRF, presumably using the same implementation $\mathcal{F}$. On the other hand, the second PRF, $\mathcal{T}$, is used to create a tag $T$ during encryption. Normally during decryption one would recompute the tag (again using $\mathcal{T}$ ) and check whether the recomputed tag $T^{\prime}$ equals the received tag $T$. Yet, in the leakage setting this approach is problematic: $T^{\prime}$ is the correct tag and its recomputation might well leak it, even when used (repeatedly) to check an incorrect and completely unrelated $T$. Hence, during decryption we will not use a recompute-and-check model, but rather refer directly to a tag-verification implementation $\mathcal{V}$ (that hopefully leaks less).

When considering the decryption leakage of $\mathrm{A} 5$, we will assume that, on invalid ciphertexts, the computation terminates as soon as the verification algorithm returns $\perp$. This implies that for invalid ciphertexts only leakage on $\mathcal{V}$ will be available, whereas for valid ciphertexts all three components $(\mathcal{V}, \mathcal{F}$, and iv $\mathcal{E})$ might leak.

Overview and interpretation. Recall that we divided the NRS schemes in three categories: MtE, M\&E, and EtM. Figure 6 shows how the composed leakage will leak for each of these schemes. For completeness, we also listed the leakage for the EtM scheme (such as A5) in case full decryption will always take place, even for invalid ciphertexts (where one could have aborted early). 


\begin{tabular}{|c|c|c|c|}
\hline Structure & Leakage & Inverse & Inverse Leakage \\
\hline $\begin{array}{l}\mathrm{MtE} \\
\mathrm{M} \& \mathrm{E}\end{array}$ & $\begin{array}{l}L_{T}(\star), L_{F}(\star), L_{E}(\star) \\
L_{T}(\star), L_{F}(\star), L_{E}(\star)\end{array}$ & DtV & $L_{F}(\star), L_{D}(\star), L_{V}(\star)$ \\
\hline & & $\mathrm{D} \& \mathrm{~V}$ & $L_{F}(\star), L_{D}(\star), L_{V}(\star)$ \\
\hline EtM & $L_{F}(\star), L_{E}(\star), L_{T}(\star)$ & $\mathrm{VtD}$ & $\begin{cases}L_{V}(\star) & \text { if } \mathcal{V}(\star)=\perp \\
L_{V}(\star), L_{F}(\star), L_{D}(\star) & \text { if } \mathcal{V}(\star)=\top\end{cases}$ \\
\hline
\end{tabular}

Fig. 6: The structure of a leakage function from a composition scheme based on the order of its primitives. The exact input parameters to the leakage function vary per scheme, so have been replaced with $\star$ : the different $\star$ variables are not the same. On the left are the encryption structures MtE, M\&E and EtM, along with the associated leakage function. The right gives the associated inverse: DtV (Decrypt then Verify) is the only way of inverting MtE or M\&E schemes. EtM schemes can be inverted in any order, as DtV, D\&V (Decrypt and Verify) or VtD (Verify then Decrypt). All constructions have the same encryption leakage, and most have the same decryption leakage. The only one that is different is an EtM-VtD scheme, where the decryption leakage format depends on the validity of the ciphertext.

Our choice to model the leakage from the authenticated encryption scheme as completely separate components from the three underlying primitives is rooted in the assumption that only computation leaks. This assumption was first formalized by Micali and Reyzin [39] and, although there are counterexamples to the assumption at for instance the gate level [47], we believe that implementations of the three primitives result in large enough physical components, which can be suitably segregated to avoid cross-leakage.

Leakage on the wire (for instance of the initial vector $I$ ) can be captured as leakage of the PRF computing the $I$ or alternatively as that of the ivE. In particular, by letting the decryption of the ivE component leak its full output (while not allowing any further leakage), we capture the release of unverified plaintext. Furthermore, distinguishable decryption failures on MtE and M\&E schemes invariably arise from verification, which might incorporate a padding check as well. This is modelled by allowing $\mathcal{V}$ to leak, but not any of the other components.

\subsection{MAC-and/then-Encrypt are Brittle under Leakage}

For schemes where the plaintext is input to the MAC (i.e. MtE and M\&E schemes), decryption is inevitably of the form DtV. Consequently, during decryption a purported message $M$ is computed before the tag can be verified. Leaking this message $M$ corresponds to the release of unverified plaintext [2], but even more modest leakage, such as the first bit of the candidate message, can be insecure as we show by the following example.

Let us assume for a moment that the encryption routine iv $\mathcal{E}$ is online, so that reencrypting a slightly modified plaintext using the same $I$ will only affect a change in the ciphertext after the modification in the plaintext. $\mathrm{CBC}$ and $\mathrm{CFB}$ modes are well-known examples of online iv $\mathcal{E}$ schemes. Additionally, assume that iv $\mathcal{E}$ 's decryption routine indeed leaks the first bit of the message. Then the authenticated encryption scheme is not secure in the presence of leakage (for the leakage class derived according to the principles outlined previously), which an attack demonstrates.

The adversary first submits a message $M$ to its challenge encryption oracle, receiving a ciphertext $C$ which either is an encryption $\mathcal{E}_{k}(M \| T)$ or, in the ideal world, a uniformly random string. The adversary subsequently queries its decryption-with-leakage oracle on $C$ with its final bit flipped. In the real world, where $C=\mathcal{E}_{k}(M \| T)$, the leakage will then equal the first bit of $M$ with probability 1 . Yet in the ideal world, $C$ is independent of $M$, so the leakage will equal the first bit of $M$ with probability half. Thus, testing whether the decryption leakage equals the first bit of $M$ leads to a distinguisher with a significant advantage. However, this does not invalidate IND-aCPLA security of iv $\mathcal{E}$ as in that game decryption only leaks on valid ciphertexts with known plaintexts.

The above observation implies that for schemes where decryption follows a DtV or D\&V structure proving generic composition secure in the presence of leakage is impossible. This affects the NRS compositions A1-A4, A7-A12, N1, N3 and N4; none of which can be regarded as generically secure under leakage and all are insecure when using online iv $\mathcal{E}$ and releasing unverified plaintext.

Less general composition results might still be possible, for instance by restricting the leakage classes of the primitives. After all, in the trivial case that the leakage classes are all $\emptyset$, the original NRS 
results hold directly. We leave open whether significantly larger realistic leakage classes exist leading to secure MtE constructions.

Alternatively, stronger assumptions on $\mathcal{E}$ could help. For instance, if $\mathcal{E}$ 's security matches that of a tweakable (variable input length) cipher, the MAC-then-Encrypt constructions become a sort of encodethen-encipher. The latter is secure against release of unverified plaintext [25]. We leave open the identification of sufficient conditions on $\mathcal{E}$ for a generic composition result in the presence of leakage to pull through for EtM or E\&M; relatedly, we leave open the extension of our work to the encode-then-encipher setting.

\subsection{Encrypt-then-MAC is Secure under Leakage}

The iv-based schemes $A 5$ and $A 6$, as well as the nonce-based N2, all fall under the EtM design. The inverse of an EtM scheme can be $\mathrm{D} \& \mathrm{~V}$ or $\mathrm{VtD}$, but as just discussed for the $\mathrm{D} \& \mathrm{~V}$ variant no meaningful generic security is possible; henceforth we restrict attention to the VtD variant only. These schemes, along with the iv $2 \mathrm{n}$ mechanism for building a nonce-based encryption scheme out of an iv-based one, are all represented in Figure 2. Before proving their security, we begin with some observations about EtM-VtD designs in the face of leakage.

Initial observations. Since the final ciphertext will be formed from an encryption ciphertext and a tag, if the overall output is to be indistinguishable from random bits, then so must the tag. Thus we require both that $(\mathcal{T}, \mathcal{V})$ is a secure $\left(\mathcal{L}_{\mathrm{T}}, \mathcal{L}_{\mathrm{V}}\right)$-LMAC, and that $\mathcal{T}$ is a secure $\mathcal{L}_{\mathrm{T}}$-LPRF. Shrimpton and Terashima [55] defined a (weaker) authenticated encryption notion where the "recovery information" does not need to be random — only the ciphertext—in which case one may drop the second requirement.

In the traditional case, it is possible to build secure EtM schemes from an encryption scheme that is IND-CPA secure. After all, by assumption on the security of the MAC, the only output the adversary can ever receive from the internal decryption function $\mathcal{D}$ is a plaintext corresponding to a previous $\mathcal{E}$ query. However, when leakage is involved, this previously harmless decryption query suddenly allows the adversary to evaluate a leakage function $L \in \mathcal{L}_{\mathrm{D}}$, albeit on a $(N, C)$ pair for which they already know the corresponding plaintext. If $\mathcal{L}_{\mathrm{D}}$ contained functions revealing sufficient information about the key, this would render the composed scheme completely broken, notwithstanding any IND-CPLA security. Luckily, the augmented IND-aCPLA game in which the adversary is allowed to leak on select decryption queries, is sufficiently nuanced to capture relevant weaknesses in the decryption's implementation.

Security of EtM composition schemes. We now describe the security of the composition schemes $\mathrm{A} 5, \mathrm{~A} 6$ and N2, and the iv2n construction. Working under the assumption of OCLI-style leakage, as described in Section 5.1, we will reduce the security of the composition to the security of its components. Technically the bound includes a term quantifying any additional weaknesses due to the composition scheme, but in all cases this term is zero. We begin with N2, and show it is essentially as secure as the weakest of its components, by constructing explicit adversaries against each.

Theorem 1 (nLAE from nLE and $\mathrm{LPRF}$ via $\mathrm{N} 2$ composition). Let $\left(\mathcal{L}_{\mathrm{E}}, \mathcal{L}_{\mathrm{D}}, \mathcal{L}_{\mathrm{T}}, \mathcal{L}_{\mathrm{V}}\right)$ be leakage sets for the appropriate primitives, and define $\left(\mathcal{L}_{\mathrm{Enc}}, \mathcal{L}_{\mathrm{Dec}}\right)$ as in Section 5.1. Let $\mathbb{A}$ be an adversary against the $\left(\mathcal{L}_{\mathrm{Enc}}, \mathcal{L}_{\mathrm{Dec}}\right)-n L A E$ security of $\mathrm{N} 2[\mathcal{E}, \mathcal{D} ; \mathcal{T}, \mathcal{V}]$. Then, there exist adversaries $\mathbb{A}_{\mathrm{CPA}}, \mathbb{A}_{\mathrm{PRF}}$ and $\mathbb{A}_{\mathrm{MAC}}$ against the $\left(\mathcal{L}_{\mathrm{E}}, \mathcal{L}_{\mathrm{D}}\right)-n L E$ security of $(\mathcal{E}, \mathcal{D})$, the $\mathcal{L}_{\mathrm{T}}-L P R F$ security of $\mathcal{T}$ and the $\left(\mathcal{L}_{\mathrm{T}}, \mathcal{L}_{\mathrm{V}}\right)$ LMAC security of $(\mathcal{T}, \mathcal{V})$ such that:

$$
\operatorname{Adv}_{\mathrm{N} 2 ; \mathcal{L}_{\mathrm{Enc}}, \mathcal{L}_{\mathrm{Dec}}}^{\mathrm{nLAE}}(\mathbb{A}) \leq \mathbf{A d v}_{\mathcal{E}, \mathcal{D} ; \mathcal{L}_{\mathrm{E}}, \mathcal{L}_{\mathrm{D}}}^{\mathrm{IND}-\mathrm{aCPLA}}\left(\mathbb{A}_{\mathrm{CPA}}\right)+\mathbf{A d v}_{\mathcal{T}_{\mathcal{T}} ; \mathcal{L}_{\mathrm{T}}}^{\mathrm{LPRF}}\left(\mathbb{A}_{\mathrm{PRF}}\right)+2 \cdot \mathbf{A d v}_{\mathcal{T}, \mathcal{V} ; \mathcal{L}_{\mathrm{T}}, \mathcal{L}_{V}}^{\mathrm{sEUF}}\left(\mathbb{A}_{\mathrm{MAC}}\right)
$$

Proof. We will begin with two identical-until-bad arguments, based on whether the adversary is able to construct a forgery against the MAC in the real or ideal worlds. If they are (and thus set the bad flag $\mathrm{F}$, for "forge"), we demonstrate how to construct an adversary against the LMAC security of the MAC. Alternatively, they cannot (and so $\neg$ F). In this case, we reduce to the IND-aCPLA security of $\mathcal{E}$, 

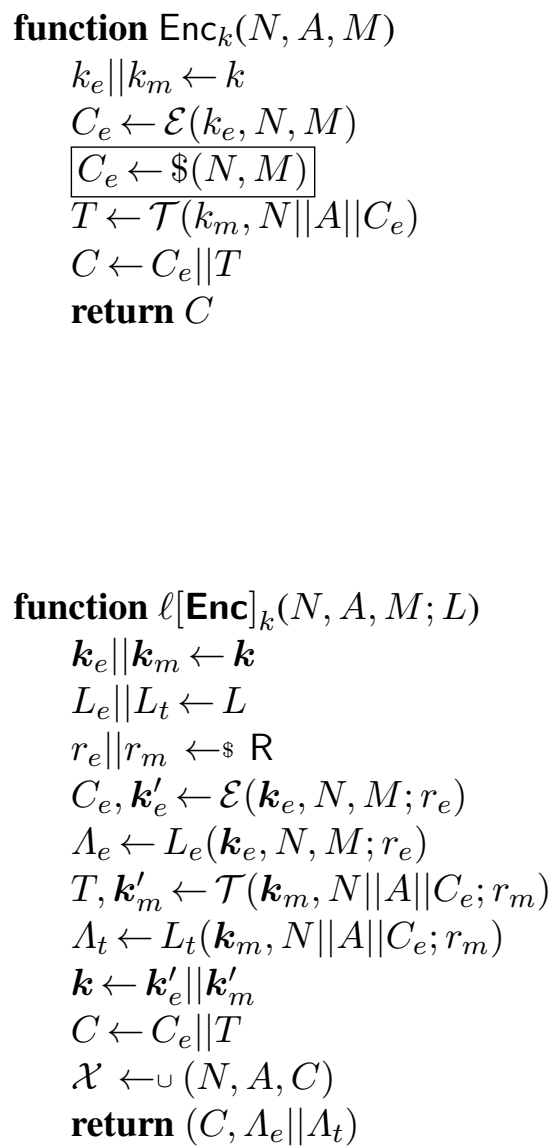

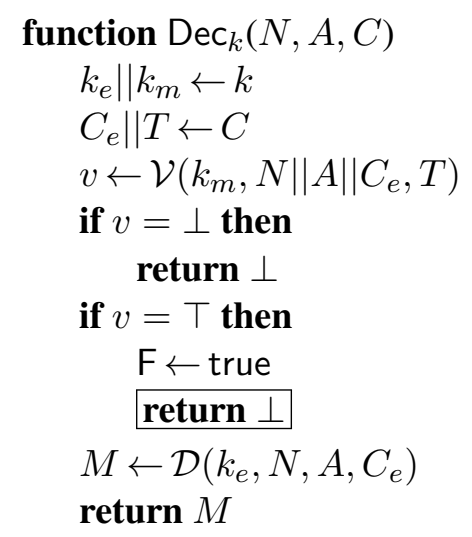

function $\ell[\mathbf{D e c}]_{k}(\boldsymbol{k}, N, A, C ; L)$

$\boldsymbol{k}_{e} \| \boldsymbol{k}_{m} \leftarrow \boldsymbol{k}$

$L_{v} \| L_{d} \leftarrow L$

$r_{e} \| r_{m} \leftarrow \$ \mathrm{R}$

$C_{e} \| T \leftarrow C$

$v, \boldsymbol{k}_{m}^{\prime} \leftarrow \mathcal{V}\left(\boldsymbol{k}_{m}, N\|A\| C_{e}, T ; r_{m}\right)$

$\Lambda_{v} \leftarrow L_{v}\left(\boldsymbol{k}_{m}, N\|A\| C_{e}, T ; r_{m}\right)$

if $v=\perp$ then

$$
\begin{aligned}
& \boldsymbol{k} \leftarrow \boldsymbol{k}_{e} \| \boldsymbol{k}_{m}^{\prime} \\
& \text { return }\left(\perp, \Lambda_{v}\right)
\end{aligned}
$$

if $v=\top \wedge(N, A, C) \notin \mathcal{X}$ then

$$
\begin{aligned}
& \mathrm{F} \leftarrow \text { true } \\
& \hline \boldsymbol{k} \leftarrow \boldsymbol{k}_{e} \| \boldsymbol{k}_{m}^{\prime} \\
& \hline \hline \text { return }\left(\perp, \Lambda_{v}\right) \\
& \hline
\end{aligned}
$$

$M, \boldsymbol{k}_{e}^{\prime} \leftarrow \mathcal{D}\left(\boldsymbol{k}_{e}, N, A, C_{e} ; r_{e}\right)$

$\Lambda_{e} \leftarrow L_{d}\left(\boldsymbol{k}_{e}, N, A, C_{e} ; r_{e}\right)$

$\boldsymbol{k} \leftarrow \boldsymbol{k}_{e}^{\prime} \| \boldsymbol{k}_{m}^{\prime}$

return $\left(M, \Lambda_{v} \| \Lambda_{d}\right)$

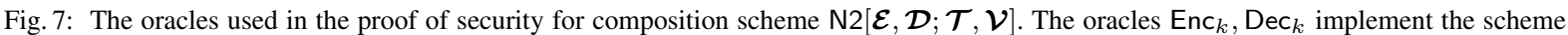
honestly and do not include the boxed code. Oracles $\ell[\mathbf{E n c}]_{k}, \ell[\mathbf{D e c}]_{k}$ implement the leakage oracle and split the leakage function following the OCLI paradigm, again not including the boxed code. In each case, including the boxed code leads to a variant used in the proof, which we denote with a box. Thus $\mathrm{Enc}_{k}$ is $\mathrm{Enc}_{k}$ with the boxed code included.

before finally using the PRLF security of $\mathcal{T}$ to complete the proof. This is formalised using the oracles of Figure 7, which implement the various functions. They also describe an alternative version by adding the boxed code. So, for example, $\ell[\mathbf{D e c}]_{k}$ contains the line "return $\perp$ ", whereas $\ell[\mathbf{D e c}]_{k}$ does not. 
So, let us start by expanding out via triangle inequality such that two of the steps are covered by trivial identical-until-bad arguments, each with bad event F:

$$
\begin{aligned}
& \mathbf{A d v}_{\mathrm{N} 2 ; \mathcal{L}_{\mathrm{Enc}}, \mathcal{L}_{\mathrm{Dec}}}^{\mathrm{LAE}}(\mathbb{A})=\underset{\mathbb{A}}{\Delta}\left(\begin{array}{c}
\mathrm{Enc}_{k}, \mathrm{Dec}_{k}, \ell[\text { Enc }]_{k}, \ell[\mathbf{D e c}]_{k} \\
\$, \perp, \ell[\mathbf{E n c}]_{k}, \ell[\mathbf{D e c}]_{k}
\end{array}\right)
\end{aligned}
$$

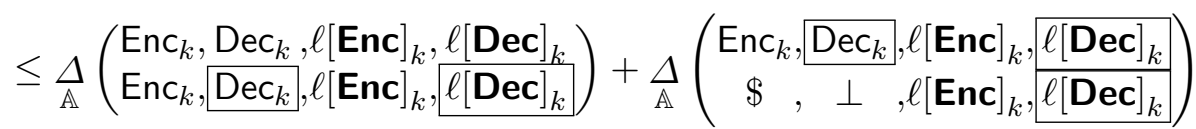

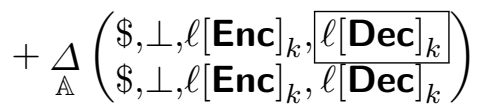

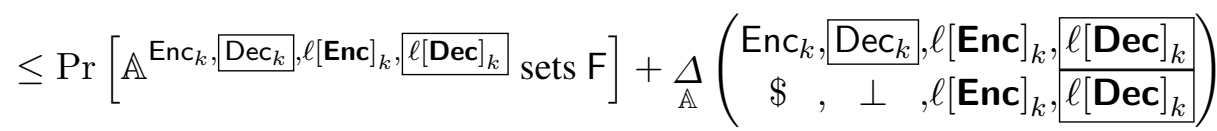

$$
\begin{aligned}
& +\operatorname{Pr}\left[\mathbb{A}^{\$, \perp, \ell[\text { Enc }]_{k},\left[\ell[\text { [Dec }]_{k}\right.} \text { sets } \mathrm{F}\right] \text {. }
\end{aligned}
$$

To bound $\operatorname{Pr}\left[\mathbb{A}^{E n c_{k}, \overline{D e c}_{k}, \ell[E n c]_{k}, \ell\left[{ }^{[D e c}\right]_{k}}\right.$ sets $\left.F\right]$, we construct an adversary $\mathbb{A}_{\text {MAC }}^{\prime}$ against the LMAC security of $(\mathcal{T}, \mathcal{V})$.

$\mathbb{A}_{\text {MAC }}^{\prime}$ draws their own key $l \leftarrow \$ \mathrm{~K}_{E}$ for the encryption scheme, and initialises an instantiation $\left(\ell[\mathcal{E}]_{l}, \ell[\mathcal{D}]_{l}\right)$ with this. As an LMAC adversary, they interact with three oracles implementing the scheme under some key $j$ : the first implements $\mathcal{V}_{j}$ or $\perp$, the other two $\ell[\mathcal{T}]_{j}$ and $\ell[\mathcal{V}]_{j}$ respectively.

Using their $\left(\ell[\mathcal{T}]_{j}, \ell[\mathcal{V}]_{j}\right)$ oracles, and $\left(\ell[\mathcal{E}]_{l}, \ell[\mathcal{D}]_{l}\right), \mathbb{A}_{\text {MAC }}^{\prime}$ simulates $\left(\right.$ Enc $\left._{k}, \operatorname{Dec}_{k}, \ell[\text { Enc }]_{k}, \ell[\text { Dec }]_{k}\right)$, and runs $\mathbb{A}$ on this simulation. Since $k$ follows the same (uniform) distribution as $l \| j$, the probability $\mathbb{A}$ sets $\mathrm{F}$ in the original setting is precisely the same as doing so in this case. If at any point a $\mathrm{F}$ is set, $\mathbb{A}_{M A C}^{\prime}$ repeats the final query to their challenge oracle, and returns 1 if the response $T$, otherwise 0 . If $\mathbb{A}$ does not set $F, \mathbb{A}_{\mathrm{MAC}}^{\prime} \rightarrow 0$.

If $\mathrm{F}$ was set by $\ell[\mathbf{D e c}]_{k}$, the query $(N, A, C) \notin \mathcal{X}$, meaning it was not output from $\ell[\text { Enc }]_{k}$, and as $\mathbb{A}$ does not forward queries from his first oracle, the query was not output from Enc either. Thus $F$ is only set by a fresh query, and so was never queried to the tagging oracle $\ell[\mathcal{T}]_{k}$ by $\mathbb{A}_{\mathrm{MAC}}^{\prime}$. Thus $\mathbb{A}_{\mathrm{MAC}}^{\prime}$ is a valid adversary, and does not make prohibited queries.

If $\mathrm{F}$ is set it must have been by some fresh query $(N, A, C)=\left(N, A, C_{e} \| T\right)$, meaning $\mathcal{V}_{j}\left(N\|A\| C_{e}, T\right)=$ $T$. So, $\mathbb{A}_{\text {MAC }}^{\prime}$ distinguishes with probability 1 . Therefore,

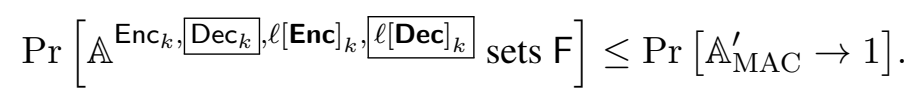

Similarly, we may construct $\mathbb{A}_{\text {MAC }}^{\prime \prime}$ to bound the other bad event. In this case we require $\mathbb{A}_{\text {MAC }}^{\prime \prime}$ to simulate the random oracle $\$$ rather than Enc, but otherwise they act equivalently. This allows us to bound

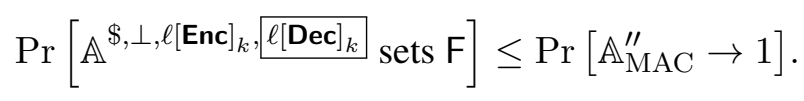

So, consider now the remaining advantage term. We observe that $\operatorname{Dec}_{k}$ returns $\perp$ to any query, and so we can bound

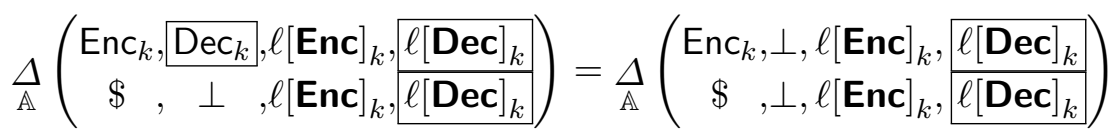

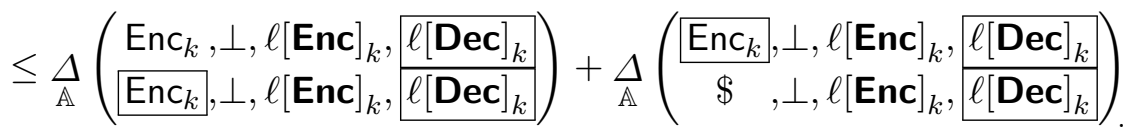

Starting with the first of these terms, we observe that the adversary only receives leakage from $\mathcal{D}_{k}$ if the final else section of $\ell[\mathbf{D e c}]_{k}$ is executed, which occurs if and only if $(N, A, C) \in \mathcal{X}$. That is, the adversary is only provided with leakage on the internal decryption function $\mathcal{D}_{k}$ if making a 


$$
\begin{aligned}
& \text { function } \mathcal{E}_{k}(N, M) \\
& \quad k_{f} \| k_{e} \leftarrow k \\
& I \leftarrow \mathcal{F}\left(k_{f}, N, M\right) \\
& I \leftarrow \$(N, M) \\
& I \leftarrow \operatorname{iv} \mathcal{E}\left(k_{e}, I, M\right) \\
& \text { return } C
\end{aligned}
$$

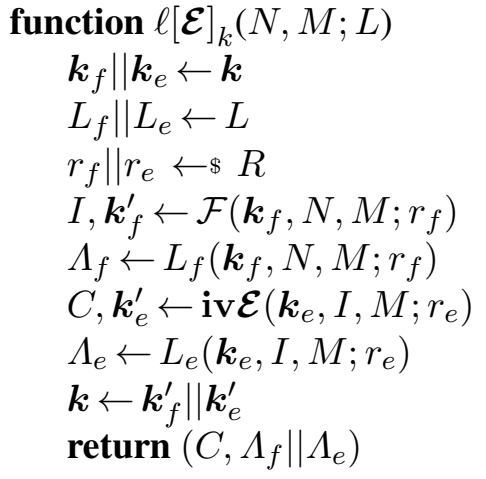

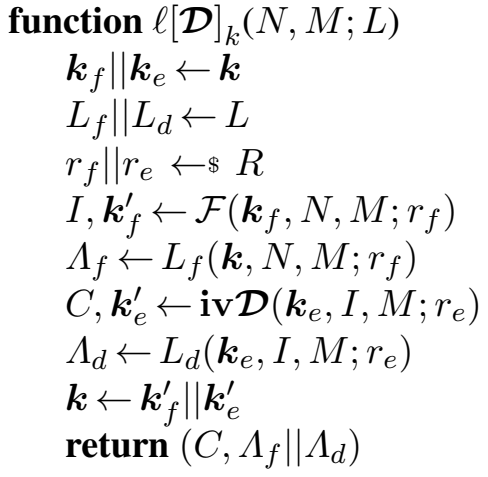

Fig. 8: Oracles from the security proof of iv2n. On the left $\mathcal{E}_{k}$ describes the scheme, and does not include the boxed code. $\mathcal{E}_{k}$, which does, is an intermediate step used in the proof. The centre and right are $\ell[\mathcal{E}]_{k}$ and $\ell[\mathcal{D}]_{k}$, with the leakage function and implementation written out in full as per their definitions. The leakage from $\ell[\mathcal{E}]_{k}$ is $L_{f}\|I\| L_{e}$, and leakage from $\ell[\mathcal{D}]_{k}$ is $L_{f}\|I\| L_{d}$.

query equivalent to a previous encryption query. We will define $\mathbb{A}_{\mathrm{CPA}}$ to be an IND-aCPLA adversary interacting with $\left(\mathcal{E}_{k}, \mathcal{D}_{k}\right)$. They will run $\mathbb{A}$ on a simulation of $\mathrm{N} 2$ and return $\mathbb{A}$ s response as their own. The composition's encryption or decryption queries are passed to $\mathbb{A}_{\mathrm{CPA}} \mathrm{s}$ corresponding oracles, and the tagging queries are run locally by picking a fresh key and verification queries are always $\perp$. By the observation above, $\mathbb{A}_{\mathrm{CPA}}$ never makes any prohibited queries to his own oracles, and so is a valid adversary.

If $\mathbb{A}_{\mathrm{CPA}}$ is in the real world and their challenge oracle is honest, the oracles they provide are precisely Enc $_{k}, \perp, \ell[\text { Enc }]_{k}, \ell[\mathbf{D e c}]_{k}$. If they are in the ideal world, then the first oracle provided to $\mathbb{A}$ is instead $E \mathrm{Cn}_{k}$. Thus they distinguish if and only if $\mathbb{A}$ does, and

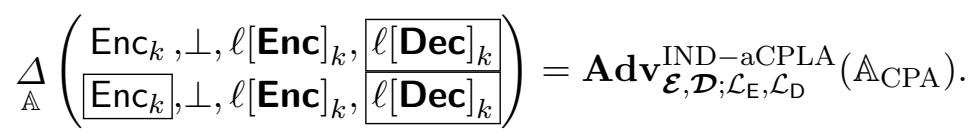

The only difference between $\$$ and $E \mathrm{Enc}_{k}$ is that in the second case the tag is not sampled uniformly at random. We construction a PRLF adversary $\mathbb{A}_{\mathrm{PRF}}$ against the PRLF security of $\mathcal{T}$ in the obvious manner. They run $\mathbb{A}$ on a simulated version of $\mathrm{N} 2$ and forward $\mathbb{A}$ s result as their own. To answer oracle queries from $\mathbb{A}, \mathbb{A}_{\mathrm{PRF}}$ samples a key and uses this to simulate all the encryption-related functionality. Queries made to $\mathcal{T}$ via the $\mathrm{Enc}_{k}$ oracle are answered by their own challenge oracle, and queries made through $\ell[\text { Enc }]_{k}$ by their own leakage oracle. Thus

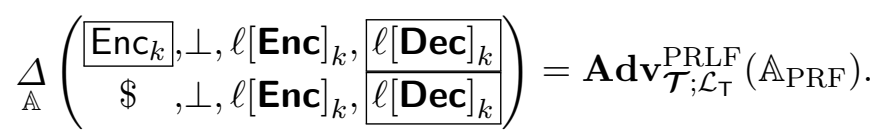

Collecting these bounds and setting $\mathbb{A}_{\mathrm{MAC}}$ to be the LMAC distinguisher with a higher distinguishing advantage (out of $\mathbb{A}_{\text {MAC }}^{\prime}$ and $\mathbb{A}_{M A C}^{\prime}$ ) gives the claimed result.

As the following result shows, the intuitive mechanism for building a nLE scheme from a secure ivLE scheme and a secure LPRF is itself secure. While unsurprising, this will allow us to instantiate the $\mathrm{N} 2$ construction with the more common object of an ivLE scheme.

Theorem 2 (nLE from ivLE and LPRF). Let $\left(\mathcal{L}_{\mathrm{ivE}}, \mathcal{L}_{\mathrm{ivD}}, \mathcal{L}_{\mathrm{F}}\right)$ be leakage sets for the appropriate primitives, and define $\left(\mathcal{L}_{\mathrm{E}}, \mathcal{L}_{\mathrm{D}}\right)$ as in Section 5.1. Let $\mathbb{A}$ be an adversary against the $\left(\mathcal{L}_{\mathrm{E}}, \mathcal{L}_{\mathrm{D}}\right)-n L E$ security of $\operatorname{iv} 2 \mathrm{n}[\mathrm{iv} \mathcal{E}, \mathrm{iv} \mathcal{D} ; \mathcal{F}]$. Then, there exist $\mathbb{A}_{\mathrm{CPA}}, \mathbb{A}_{\mathrm{PRF}}$ against the $\left(\mathcal{L}_{\mathrm{ivE}}, \mathcal{L}_{\mathrm{ivD}}\right)$-ivLE security of (iv $\mathcal{E}$, iv $\mathcal{D}$ ), and the $\mathcal{L}_{\mathrm{F}}-L P R F$ security of $\mathcal{F}$ respectively, such that:

$$
\mathbf{A d v}_{\mathrm{iv} 2 \mathrm{n} ; \mathcal{L}_{\mathrm{E}}, \mathcal{L}_{\mathrm{D}}}^{\mathrm{IND}-\mathrm{aCPLA}}(\mathbb{A}) \leq \mathbf{A d v}_{\mathcal{F} ; \mathcal{L}_{\mathrm{F}}}^{\mathrm{LPRF}}\left(\mathbb{A}_{\mathrm{PRF}}\right)+\mathbf{A d v}_{\mathbf{i v} \mathcal{E}, \mathrm{iv} \mathcal{D} ; \mathcal{L}_{\text {ive }}, \mathcal{L}_{\mathrm{ivD}}}^{\mathrm{IND}}\left(\mathbb{A}_{\mathrm{CPA}}\right)
$$


Proof. By triangle inequality,

$$
\operatorname{Adv}_{\mathrm{iv2n} ; \mathcal{L}_{\mathrm{E}}, \mathcal{L}_{\mathrm{D}}}^{\mathrm{IND}}(\mathbb{A})=\underset{\mathbb{A}}{\Delta}\left(\begin{array}{c}
\mathcal{E}_{k}, \ell[\mathcal{E}]_{k}, \ell[\mathcal{D}]_{k} \\
\$, \ell[\mathcal{E}]_{k}, \ell[\mathcal{D}]_{k}
\end{array}\right) \leq \underset{\mathbb{A}}{\Delta}\left(\begin{array}{c}
\mathcal{E}_{k}, \ell[\mathcal{E}]_{k}, \ell[\mathcal{D}]_{k} \\
\mathcal{E}_{k}, \ell[\mathcal{E}]_{k}, \ell[\mathcal{D}]_{k}
\end{array}\right)+\underset{\mathbb{A}}{\Delta}\left(\begin{array}{c}
\mathcal{E}_{k}, \ell[\mathcal{E}]_{k}, \ell[\mathcal{D}]_{k} \\
\$, \ell[\mathcal{E}]_{k}, \ell[\mathcal{D}]_{k}
\end{array}\right)
$$

Let $\mathbb{A}_{\mathrm{PRF}}$ be a PRLF adversary against $\mathcal{F}$. She runs $\mathbb{A}$ on a simulated version of the construction. To answer $\mathbb{A}$ s challenge queries, they use their challenge oracle for $\mathcal{F}$ and a random key to instantiate iv $\mathcal{E}$. Since the leakage sets follow the OCLI paradigm, any leakage function $L_{f}$ is a valid query to make to their own leakage oracle, so the first half of the leakage oracle can be simulated using their own leakage oracles. For the second half of the leakage oracles, they use their iv $\mathcal{E}$ key to evaluate iv $\mathcal{E}$, iv $\mathcal{D}$ and $L_{e}$. Finally, they forward $\mathbb{A} s$ answer as their own. If their challenge oracle is honest, this perfectly matches $\left(\mathcal{E}_{k}, \ell[\mathcal{E}]_{k}, \ell[\mathcal{D}]_{k}\right)$, whereas if their challenge oracle is ideal this exactly matches $\left(\mathcal{E}_{k}, \ell[\mathcal{E}]_{k}, \ell[\mathcal{D}]_{k}\right)$. Thus,

$$
\underset{\mathbb{A}}{\Delta}\left(\begin{array}{c}
\mathcal{E}_{k}, \ell[\mathcal{E}]_{k}, \ell[\mathcal{D}]_{k} \\
\mathcal{E}_{k}, \ell[\mathcal{E}]_{k}, \ell[\mathcal{D}]_{k}
\end{array}\right)=\operatorname{Adv}_{\mathcal{F} ; \mathcal{L}_{\mathrm{F}}}^{\mathrm{PRLF}}\left(\mathbb{A}_{\mathrm{PRF}}\right)
$$

Conversely, let $\mathbb{A}_{\mathrm{CPA}}$ be an adversary against the $\left(\mathcal{L}_{\mathrm{ivE}}, \mathcal{L}_{\mathrm{ivD}}\right)$-ivLE security of (iv $\mathcal{E}$, iv $\left.\mathcal{D}\right)$. He runs $\mathbb{A}$, answering any encryption-related queries with his own oracles, and evaluating the rest locally. Then, he forwards $\mathbb{A}$ s response as his own. At no point does $\mathbb{A}$ ask a query that he is himself forbidden from asking his oracles, and the leakage sets $\left(\ell[\mathcal{E}]_{k}, \ell[\mathcal{D}]_{k}\right)$ follow the OCLI paradigm, meaning any leakage queries he forwards are valid. Moreover, since the variable $I$ is randomly sampled, he is an iv-respecting adversary. The fact $\mathbb{A}$ has access to $I$, the IV, through leakage does not prevent this since it is only available after the fact, and not in advance. In the real world, the oracles he proves $\mathbb{A}$ match $\mathcal{E}_{k}, \ell[\mathcal{E}]_{k}, \ell[\mathcal{D}]_{k}$, and in the ideal case they match $\$, \ell[\mathcal{E}]_{k}, \ell[\mathcal{D}]_{k}$. Thus,

$$
\underset{\mathbb{A}}{\Delta}\left(\frac{\mathcal{E}_{k}, \ell[\mathcal{E}]_{k}, \ell[\mathcal{D}]_{k}}{S, \ell[\mathcal{E}]_{k}, \ell[\mathcal{D}]_{k}}\right)=\mathbf{A d v}_{\text {iv } \mathcal{E}, \text { iv } \mathcal{D} ; \mathcal{L}_{\text {ive }}, \mathcal{L}_{\text {ivD }}}^{\text {IND }}\left(\mathbb{A}_{\mathrm{CPA}}\right)
$$

Substituting these into the above expansion competes the proof.

Pulling these two results together and taking the maximum over the similar adversaries, we are able to prove the security of the $\mathrm{A} 5$ construction. The security of $\mathrm{A} 6$ against adversaries who never repeat the pair $(N, A)$ can be easily recovered from this by considering it as an equivalent representation of the $\mathrm{A} 5$ scheme acting on nonce space $\mathrm{N}^{\prime}=\mathrm{N} \times \mathrm{A}$ but with no associated data.

Corollary 1 (nLAE from ivLE and LPRF via A5 composition). Let $\left(\mathcal{L}_{\mathrm{ivE}}, \mathcal{L}_{\mathrm{ivD}}, \mathcal{L}_{\mathrm{T}}, \mathcal{L}_{\mathrm{V}}, \mathcal{L}_{\mathrm{F}}\right)$ be leakage sets for the appropriate primitives, and define $\left(\mathcal{L}_{\mathrm{Enc}}, \mathcal{L}_{\mathrm{Dec}}\right)$ as in Section 5.1. Let $\mathbb{A}$ be an adversary against the $\left(\mathcal{L}_{\text {Enc }}, \mathcal{L}_{\text {Dec }}\right)$-nLAE security of $\mathrm{A} 5[\mathrm{iv} \mathcal{E}, \mathrm{iv} \mathcal{D} ; \mathcal{F} ; \mathcal{T}, \mathcal{V}]$. Then, there exist adversaries $\mathbb{A}_{\mathrm{CPA}}$, $\mathbb{A}_{\mathrm{PRF}}, \mathbb{A}_{\mathrm{PRF}}^{\prime}$, and $\mathbb{A}_{\mathrm{MAC}}$ against the $\left(\mathcal{L}_{\mathrm{ivE}}, \mathcal{L}_{\mathrm{ivD}}\right)$-ivLE security of (iv $\left.\mathcal{E}, \mathrm{iv} \mathcal{D}\right)$, the $\mathcal{L}_{\mathrm{F}}-L P R F$ security of $\mathcal{F}$, the $\mathcal{L}_{\mathrm{T}}-L P R F$ security of $\mathcal{T}$ and the $\left(\mathcal{L}_{\mathrm{T}}, \mathcal{L}_{\mathrm{V}}\right)$-LMAC security of $(\mathcal{T}, \mathcal{V})$ such that

$$
\begin{aligned}
\operatorname{Adv}_{\mathrm{A} 5 ; \mathcal{L}_{\text {Enc }}, \mathcal{L}_{\text {Dec }}}^{\mathrm{nLAE}}(\mathbb{A}) \leq & \mathbf{A d v}_{\mathcal{E}, \mathcal{D} ; \mathcal{L}_{\text {ive }}, \mathcal{L}_{\text {ivD }}}^{\mathrm{IND}}\left(\mathbb{A}_{\mathrm{CPA}}\right)+\operatorname{Adv}_{\mathcal{F} ; \mathcal{L}_{\mathrm{F}}}^{\mathrm{LPRF}}\left(\mathbb{A}_{\mathrm{PRF}}\right) \\
& +\operatorname{Adv}_{\mathcal{T} ; \mathcal{L}_{\mathrm{T}}}^{\mathrm{LPRF}}\left(\mathbb{A}_{\mathrm{PRF}}^{\prime}\right)+2 \cdot \mathbf{A d v}_{\mathcal{T}, \mathcal{V} ; \mathcal{L}_{\mathrm{T}}, \mathcal{L}_{\mathrm{V}}}^{\mathrm{EUF}-\mathrm{CMLA}}\left(\mathbb{A}_{\mathrm{MAC}}\right) .
\end{aligned}
$$

Corollary 2 (nLAE from ivLE and LPRF via $\mathrm{A} 6$ composition). Let $\left(\mathcal{L}_{\mathrm{ivE}}, \mathcal{L}_{\mathrm{ivD}}, \mathcal{L}_{\mathrm{T}}, \mathcal{L}_{\mathrm{V}}, \mathcal{L}_{\mathrm{F}}\right)$ be leakage sets for the appropriate primitives, and define $\left(\mathcal{L}_{\mathrm{Enc}}, \mathcal{L}_{\mathrm{Dec}}\right)$ as in Section 5.1. Let $\mathbb{A}$ be an adversary against the $\left(\mathcal{L}_{\text {Enc }}, \mathcal{L}_{\text {Dec }}\right)-L A E$ security of $\mathrm{A} 6[\mathrm{iv} \mathcal{E}, \mathrm{iv} \mathcal{D} ; \mathcal{F} ; \mathcal{T}, \mathcal{V}]$ who does not make two encryption queries with the same $(N, A)$ pair. Then, there exist explicit adversaries $\mathbb{A}_{\mathrm{CPA}}, \mathbb{A}_{\mathrm{PRF}}, \mathbb{A}_{\mathrm{PRF}}^{\prime}$, and $\mathbb{A}_{\mathrm{MAC}}$ against the $\left(\mathcal{L}_{\mathrm{ivE}}, \mathcal{L}_{\mathrm{ivD}}\right)$-ivLE security of (iv $\left.\mathcal{E}, \mathrm{iv} \mathcal{D}\right)$, the $\mathcal{L}_{\mathrm{F}}-L P R F$ security of $\mathcal{F}$, the $\mathcal{L}_{\mathrm{T}}-L P R F$ security of $\mathcal{T}$ and the $\left(\mathcal{L}_{\mathrm{T}}, \mathcal{L}_{\mathrm{V}}\right)-L M A C$ security of $(\mathcal{T}, \mathcal{V})$ such that

$$
\begin{aligned}
\operatorname{Adv}_{\mathrm{A} 6 ; \mathcal{L}_{\text {Enc }}, \mathcal{L}_{\text {Dec }}}^{\mathrm{nLAE}}(\mathbb{A}) \leq & \mathbf{A d v}_{\mathcal{E}, \mathcal{D} ; \mathcal{L}_{\text {ive }}, \mathcal{L}_{\text {iv }}}^{\mathrm{IND}-\mathrm{aCPLA}}\left(\mathbb{A}_{\mathrm{CPA}}\right)+\operatorname{Adv}_{\mathcal{F} ; \mathcal{L}_{\mathrm{F}}}^{\mathrm{LPRF}}\left(\mathbb{A}_{\mathrm{PRF}}\right) \\
& +\operatorname{Adv}_{\mathcal{T} ; \mathcal{L}_{\mathrm{T}}}^{\mathrm{LPRF}}\left(\mathbb{A}_{\mathrm{PRF}}^{\prime}\right)+2 \cdot \mathbf{A d v}_{\mathcal{T}, \mathcal{V} ; \mathcal{L}_{\mathrm{T}}, \mathcal{L}_{V}}^{\mathrm{sEUF}}\left(\mathbb{A}_{\mathrm{MAC}}\right)
\end{aligned}
$$




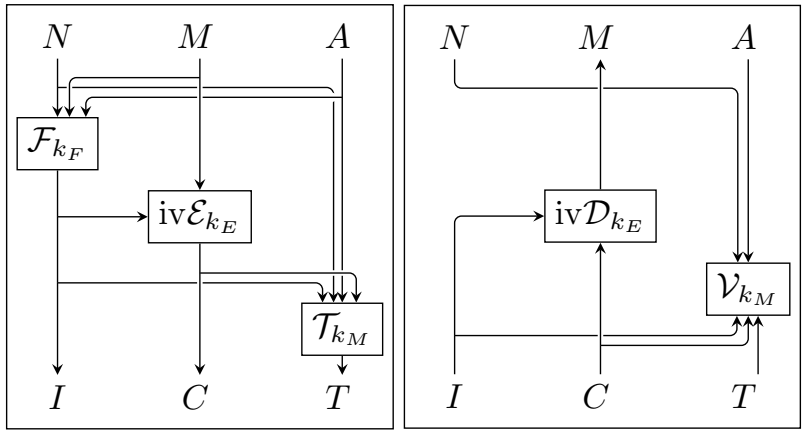

Fig. 9: The Synthetic-IV-and-Tag (SIVAT) scheme. On the left, the encryption routine runs from top to bottom, outputting a ciphertext $I\|C\| T$. Decryption (on the right) runs from bottom to top. If during decryption verification fails, and $\mathcal{V}_{\boldsymbol{k}_{m}}$ returns $\perp$, no further computations are performed. In the decryption direction, the PRF $\mathcal{F}$ is not required.

\subsection{Achieving Misuse Resistant LAE Security}

In Section 5.2 we discussed why no composition scheme can be (generically) secure against leakage if its decryption begins by calculating a candidate plaintext. This meant ruling out every NRS construction secure in the nonce misuse model, an important feature for a modern robust AE schemes [10,25,51]. Roughly speaking, for MRAE security a scheme must be MtE (to ensure maximum diffusion) yet for leakage resilience it must be EtM (to ensure minimal leakage).

The Synthetic IV and Tag (SIVAT) scheme, defined in Figure 9, addresses the combined mrLAE goal, by essentially using an MtEtM approach. It can be seen as composing the SIV construction [51] (referred to as A4 in NRS) with a secure MAC, or alternatively as the natural strengthening of A6 towards nonce misuse security, by adding the message to the IV calculation and making the appropriate modifications to enable decryption.

Our additional feature does come at a cost. While schemes in the traditional setting achieve misuse resistance for the same ciphertext expansion as non-resistant schemes, the SIVAT scheme requires essentially twice the expansion. It also has a large number of internal wires, with each function taking in a large number of inputs, although removing any one leads to incorrectness or insecurity. For encryption calls, all inputs must go into the LPRF (for misuse resistance) and for decryption they must go into verification (to prevent RUP attacks).

The proof is very similar to that for $A 5$ or $A 6$ (Corollaries 1 and 2), since the additional element of a SIVAT ciphertext $(I)$ is present in those settings, and might already be available to the adversary through leakage.

Theorem 3 (mrLAE from ivLE and LPRF via SIVAT composition). Let $\left(\mathcal{L}_{\mathrm{ivE}}, \mathcal{L}_{\mathrm{ivD}}, \mathcal{L}_{\mathrm{T}}, \mathcal{L}_{\mathrm{V}}, \mathcal{L}_{\mathrm{F}}\right)$ be leakage sets for the appropriate primitives, and define $\left(\mathcal{L}_{\mathrm{Enc}}, \mathcal{L}_{\mathrm{Dec}}\right)$ as in Section 5.1. Let $\mathbb{A}$ be an adversary against the $\left(\mathcal{L}_{\mathrm{Enc}}, \mathcal{L}_{\text {Dec }}\right)$-mrLAE security of $\operatorname{SIVAT}[\mathrm{iv} \mathcal{E}, \mathrm{iv} \mathcal{D} ; \mathcal{F} ; \mathcal{T}, \mathcal{V}]$. Then, there exist explicit adversaries $\mathbb{A}_{\mathrm{CPA}}, \mathbb{A}_{\mathrm{PRF}}, \mathbb{A}_{\mathrm{PRF}}^{\prime}$, and $\mathbb{A}_{\mathrm{MAC}}$ against the $\left(\mathcal{L}_{\mathrm{ivE}}, \mathcal{L}_{\mathrm{ivD}}\right)$-ivLE security of (ive $\mathcal{E}$, iv $\left.\mathcal{D}\right)$, the $\mathcal{L}_{\mathrm{F}}-L P R F$ security of $\mathcal{F}$, the $\mathcal{L}_{\mathrm{T}}-L P R F$ security of $\mathcal{T}$ and the $\left(\mathcal{L}_{\mathrm{T}}, \mathcal{L}_{\mathrm{V}}\right)-L M A C$ security of $(\mathcal{T}, \mathcal{V})$ such that

$$
\begin{aligned}
& \mathbf{A d v}_{\mathrm{SIVAT} ; \mathcal{L}_{\mathrm{Enc}}, \mathcal{L}_{\text {Dec }}^{\mathrm{nLLAE}}}(\mathbb{A}) \leq \mathbf{A d} \mathbf{v}_{\mathcal{E}, \mathcal{D} ; \mathcal{L}_{\mathrm{ivE}}, \mathcal{L}_{\mathrm{ivD}}}^{\mathrm{IND}-\mathrm{aCPLA}}\left(\mathbb{A}_{\mathrm{CPA}}\right)+\mathbf{A d v}_{\mathcal{F} ; \mathcal{L}_{\mathrm{F}}}^{\mathrm{LPRF}}\left(\mathbb{A}_{\mathrm{PRF}}\right) \\
& +\operatorname{Adv}_{\mathcal{T} ; \mathcal{L}_{T}}^{\mathrm{LPRF}}\left(\mathbb{A}_{\mathrm{PRF}}^{\prime}\right)+2 \cdot \mathbf{A d v}_{\mathcal{T}, \mathcal{V} ; \mathcal{L}_{\mathrm{T}}, \mathcal{L}_{V}}^{\mathrm{sEUF}}\left(\mathbb{A}_{\mathrm{MAC}}\right) .
\end{aligned}
$$

Proof. We immediately observe that the SIVAT construction contains no wires that are not given to the adversary as output, or themselves inputs. Thus the leakage can be expressed very succinctly as leakage of the internal primitives alone, (along with the order in which this occurs). With this in mind, the proof proceeds in a similar, manner to that of Theorems 1 and 2 above, if anything being simpler. Let $\mathbb{A}$ be an adversary against the mrLAE security of SIVAT, and recall that this means he never repeats the triple $(N, A, M)$ on an encryption query, nor forwards queries to/from his challenge oracles.

We begin almost identically to the proof of Theorem 1, performing an identical-until-bad switch on both real and ideal worlds. We define the event $F$ as that of the adversary making a second or 


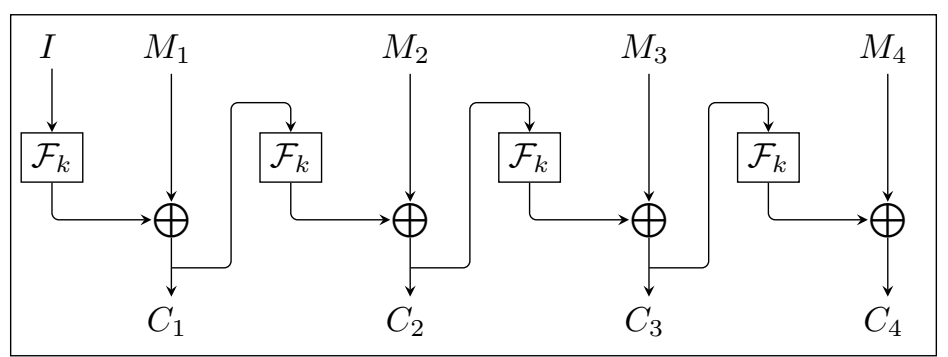

Fig. 10: CFB Mode of Operation based on $\mathcal{F}: \mathrm{K} \times \mathrm{X} \rightarrow \mathrm{X}$. The message $M$ is parsed into blocks or elements $M_{1}\|\ldots\| M_{m}$, and fed through to output ciphertext $C_{1}\|\ldots\| C_{m}$. The operation $\oplus$ can be any group operation on $\mathrm{X}$.

fourth oracle query $\left(N, A, I\left\|C_{e}\right\| T\right)$ that is not the result of a previous encryption query but that $\mathcal{V}_{k_{m}}\left(N, A, I\left\|C_{e}\right\| T\right)=\top$. By the same logic as before exist explicit adversaries $\mathbb{A}_{\mathrm{MAC}}$ and $\mathbb{A}_{\mathrm{MAC}}^{\prime}$ such that the probability the adversary $\mathbb{A}$ triggering $\mathrm{F}$ when interacting with either the real or ideal world in either setting is less than that of the appropriate adversary winning the EUF-CMLA game.

Next, define an adversary against the PRLF security of $\mathcal{F}$, analogous to that in Theorem 2 . They build the SIVAT construction around their own challenge and leakage oracles, instantiated the ivE and tagging schemes with internally sampled primitives: after the previous switch verification can be idealised to reject all non-forwarded queries. They run $\mathbb{A}$ on this, and output $\mathbb{A}$ s result as their own. Since $\mathbb{A}$ never repeats a triple $(N, A, M)$, every query made to the PRF is unique, making $\mathbb{A}_{\mathrm{PRF}}$ a valid PRF adversary. So, $\mathbb{A}$ can distinguish whether $\mathcal{F}$ has been replaced with an idealised version if and only if $\mathbb{A}_{\mathrm{PRF}}$ wins the PRLF game.

Third, we replace $\mathcal{E}$ with the idealised form $\$$. As with Theorem 1, we construct the adversary $\mathbb{A}_{\mathrm{CPA}}$ against the IND-aCPLA security of $(\mathcal{E}, \mathcal{D})$ who builds this scheme by choosing the other primitives himself, runs $\mathbb{A}$ on it and forwards $\mathbb{A}$ s result as his own. He is able to do this because the only decryption queries that call $\mathcal{D}$ or $\ell[\mathcal{D}]_{k}$ are those which are repeats of previous queries, due to the earlier switch of $\mathcal{V}$. Then $\mathbb{A}$ distinguishes between these cases if and only if $\mathbb{A}_{\text {CPA }}$ wins the IND-aCPLA game.

Finally, we construct an adversary $\mathbb{A}_{\text {PRF }}^{\prime}$ against the PRLF security of $\mathcal{T}$, who simply runs $\mathbb{A}$ on an implementation of SIVAT in which every component has been idealised other than $\mathcal{T}$ and its associated leakage. This completes a chain of game hops which have taken us from the real to the ideal world, leaving just to collect the terms to form the final bound.

\subsection{A Leakage Resilient IV-based Encryption Scheme}

A crucial component required for our composition is an encryption scheme iv $\mathcal{E}$, whose implementation $(\mathbf{i v} \mathcal{E}, \mathbf{i v} \mathcal{D})$ is IND-aCPLA secure against a rich class $\left(\mathcal{L}_{\text {ivE }}, \mathcal{L}_{\text {ivD }}\right)$ of leakage functions. As generic composition relies on a secure PRLF implementation $\mathcal{F}$ anyway, we will investigate to what extent this PRLF can be used to bootstrap some ivE implementation as well. Here we turn to the classical mode of operation CFB (Fig. 10), which has the advantage that only the forward direction of the underlying primitive $\mathcal{F}$ is required, even for decryption (relevant if one would instantiate with a blockcipher). When we move from the standard $\mathrm{CFB}[\mathcal{F}]$ to its implementation $\mathrm{CFB}[\mathcal{F}]$ (by replacing $\mathcal{F}$ with its implementation $\mathcal{F}$ ), processing multi-block plaintexts (or ciphertexts) will result in multiple refreshes of the key's representation $\boldsymbol{k}$ (one for each call to $\mathcal{F}$ ). We will show that CFB is secure against leakage when instantiated with a PRLF, using an adaptation of the classical proof for CFB security [1].

Our first task is to express the leakage sets $\left(\mathcal{L}_{\mathrm{ivE}}, \mathcal{L}_{\mathrm{ivD}}\right)$ for scheme iv $\mathcal{E}$ in terms of that of the PRF $\mathcal{F}$, namely $\mathcal{L}_{\mathrm{F}}$. When tracing through the operation of CFB-encryption, we will make two assumptions. Firstly, that leakage for each of the $\mathcal{F}$ calls is local (cf. OCLI), which in particular means leakage will be restricted to the representation of $\boldsymbol{k}$ specific for the $\mathcal{F}$ call at hand (and $\boldsymbol{k}$ is expected to be refreshed during a single iv $\mathcal{E}$ call). Secondly, that all visible wires in Fig. 10, corresponding to the iv $\mathcal{E}$ 's public inputs and outputs, will leak. Note that longer messages will lead to more leakage for an adversary, which matches practice (where the size of the power trace might be linear in the size of the message.

Decryption closely matches encryption and, under the same assumptions as above, leakage on decryption of a ciphertext can be expressed instead as leakage on the encryption of the corresponding plain- 


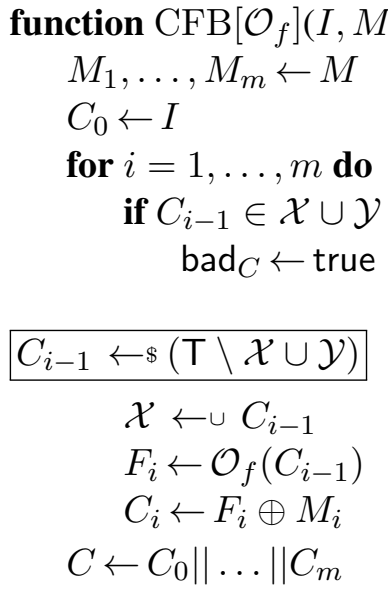

return $C$

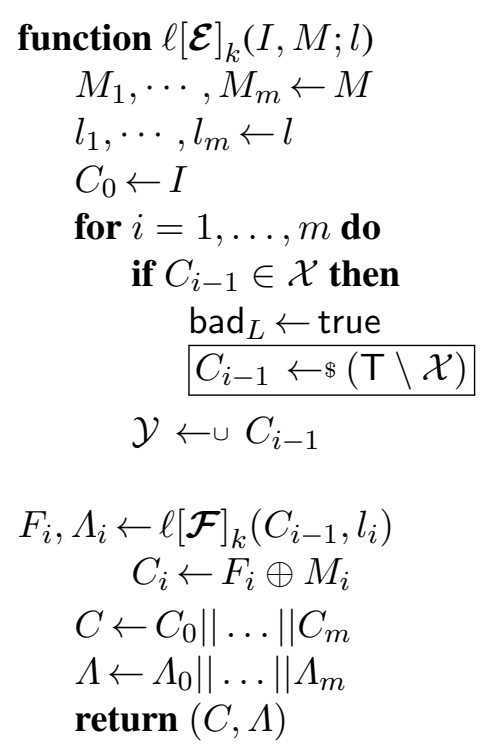

$C_{0}, \cdots, C_{m} \leftarrow C$

$l_{1}, \cdots, l_{m} \leftarrow l$

for $i=1, \ldots, m$ do

$$
\begin{gathered}
F_{i}, \Lambda_{i} \leftarrow \ell[\mathcal{F}]_{k}\left(C_{i-1}, l_{i}\right) \\
M_{i} \leftarrow F_{i} \oplus C_{i}
\end{gathered}
$$$$
M \leftarrow M_{0}, \ldots, M_{m}
$$$$
\Lambda \leftarrow \Lambda_{0}, \ldots, \Lambda_{m}
$$

return $(M, \Lambda)$

Fig. 11: Three oracles used to bound the IND-aCPLA advantage against $\mathrm{CFB}[\mathcal{F}]$. The first, $\mathrm{CFB}\left[\mathcal{O}_{f}\right]$ is $\mathrm{CFB}$ mode instantiated around some function $\mathcal{O}_{f}$ and does not include the boxed code. It also shows $\mathrm{CFB}\left[\mathcal{O}_{f}\right]$, which does include the boxed code. When $\mathcal{O}_{f}=\mathcal{F}_{k}$, $\mathrm{CFB}\left[\mathcal{F}_{k}\right]=\mathcal{E}_{k}$. The second and third panels show $\ell[\mathcal{E}]_{k}$ and $\ell[\mathcal{D}]_{k}$. The list $\mathcal{X}$ tracks all blocks queried to $\mathcal{F}_{k}$, and $\mathcal{Y}$ tracks blocks that have been queried to $\ell[\mathcal{F}]_{k}$.

text. Hence we refer to decryption leakage as $\mathcal{L}_{\text {ivE }}$ (where the prime connotes the syntactical malarkey to deal with the different input spaces for encryption and decryption).

Concluding, we define the leakage set $\mathcal{L}_{\text {ivE }}$ to be the collection of all functions $L_{\mathrm{CFB}}: \mathbf{K} \times \mathrm{N} \times \mathrm{M} \times$ $\mathrm{R} \rightarrow\{0,1\}^{*}$ that are of the form

$$
L_{\mathrm{CFB}}(\boldsymbol{k}, I, M ; r)=\left(M, C, L_{i}\left(\boldsymbol{k}_{i}, C_{i} ; r_{i}\right)_{i \in\{0 . . n-1\}}\right)
$$

with $L_{i} \in \mathcal{L}_{\mathrm{T}}$ (for $i \in\{0 . . n-1\}$ ) and where $M$ is an $n$-block message, $C=$ iv $\mathcal{E}_{k}(I, M)$ is an $(n+1)$ block ciphertext constituted of blocks $C_{i}(i \in\{0, . . n\}), r$ is the concatenation of the random values $r_{i}$ passed to the $i^{\text {th }} \mathcal{F}$-call $(i \in\{1 . . n\})$, and $\boldsymbol{k}_{i-1}$ is the key representation for the $i^{\text {th }} \mathcal{F}$-call $(i \in\{1 . . n\})$.

Theorem 4 (ivLE from LPRF via CFB mode). Let $\mathcal{F}: \mathrm{T}^{*} \rightarrow \mathrm{T}$ be a PRF with leakage class $\mathcal{L}_{\mathrm{F}}$ and let (iv $\mathcal{E}, \mathrm{iv} \mathcal{D})$ be the symmetric encryption scheme $\mathrm{CFB}[\mathcal{F}]$ with derived leakage $\left(\mathcal{L}_{\mathrm{ivE}}, \mathcal{L}_{\mathrm{ivE}}{ }^{\prime}\right)$. Let $\mathbb{A}$ be an iv-respecting adversary against the $\left(\mathcal{L}_{\mathrm{ivE}}, \mathcal{L}_{\mathrm{ivE}}\right)^{\prime}-I N D-a C P L A$ security of $(\mathrm{iv} \mathcal{E}, \mathrm{iv} \mathcal{D})$. Then there exists an adversary $\mathbb{A}_{\mathrm{PRF}}$ of similar complexity to $\mathbb{A}$ against the $\mathcal{L}_{\mathrm{F}}-P R L F$ security of $\mathcal{F}$ such that

$$
\operatorname{Adv}_{\mathbf{i v} \mathcal{E}, \text { iv } \mathcal{D} ; \mathcal{L}_{\mathrm{ivE}}, \mathcal{L}_{\mathrm{ivE}}}^{\mathrm{IND}}(\mathbb{A}) \leq 2 \cdot \mathbf{A d v}_{\mathcal{F} ; \mathcal{L}_{\mathrm{F}}}^{\mathrm{PRLF}}\left(\mathbb{A}_{\mathrm{PRF}}\right)+\frac{3}{4} \cdot \frac{\sigma^{2}}{|\mathrm{~T}|}
$$

where $\sigma$ is the total number of blocks encrypted, and the blocksize is $|\mathrm{T}|$.

Proof (Of Theorem 4). We will use a code-based proof method, with oracles provided in Figure 11. As noted in the caption of the figure, $\mathcal{E}_{k}=\mathrm{CFB}\left[\mathcal{F}_{k}\right]$. The function $\$^{\prime}: \mathrm{T} \rightarrow \mathrm{T}$ is a random function.

With these definitions set, we begin by bounding

$$
\begin{aligned}
& \mathbf{A d v}_{\text {iv } \mathcal{E}, \text { iv } \mathcal{D} ; \mathcal{L}_{\text {ive }}, \mathcal{L}_{\text {ive }}}^{\mathrm{IND}}(\mathbb{A})=\underset{\mathbb{A}}{\Delta}\left(\begin{array}{r}
\mathrm{CFB}\left[\mathcal{F}_{k}\right], \ell[\mathcal{E}]_{k}, \ell[\mathcal{D}]_{k} \\
\$, \ell[\mathcal{E}]_{k}, \ell[\mathcal{D}]_{k}
\end{array}\right)
\end{aligned}
$$

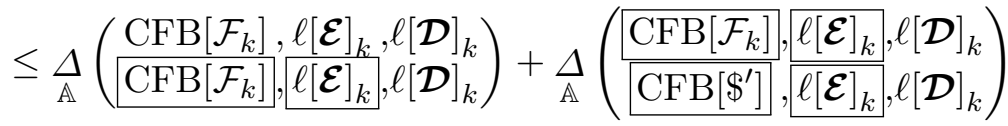

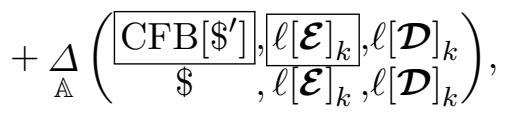


three terms we now bound individually.

Consider the first term in the expanded bound. By their construction, $\mathcal{E}_{k}$ and $\overline{\mathcal{E}_{k}}$ are identical-until-

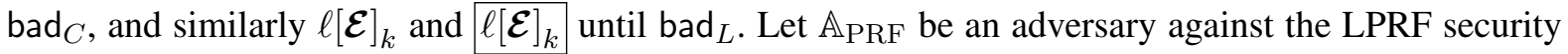
of $\mathcal{F}$, with challenge oracle $\mathcal{O}_{C}$. He runs $\mathbb{A}$, answering $\mathbb{A}$ 's first oracle queries with $\mathrm{CFB}\left[\mathcal{O}_{C}\right]$, which $\mathbb{A}_{\mathrm{PRF}}$ evaluates locally using his own challenge oracle. Any leakage queries are answered by simulating $\ell[\mathcal{E}]_{k}, \ell[\mathcal{D}]_{k}$ with his own leakage oracles. If $\mathbb{A}$ sets bad $=\operatorname{bad}_{C} \vee \operatorname{bad}_{L}$, then $\mathbb{A}_{\mathrm{PRF}} \rightarrow 1$; otherwise $\mathbb{A}_{\mathrm{PRF}} \rightarrow 0$. Note that $\mathbb{A}_{\mathrm{PRF}}$ never makes any prohibited queries to his oracles, since he only queries while $\neg$ bad, at which point all PRF queries are either fresh, or a leakage query repeating a previous leakage query.

Then,

$$
\begin{aligned}
& \operatorname{Pr}\left[\mathbb{A}_{\mathrm{PRF}}^{\mathcal{F}_{k}, \ell[\mathcal{F}]_{k}} \rightarrow 1\right]=\operatorname{Pr}\left[\mathbb{A}^{\mathrm{CFB}\left[\mathcal{F}_{k}\right], \ell[\mathcal{E}]_{k}, \ell[\mathcal{D}]_{k}} \text { sets bad }\right], \\
& \operatorname{Pr}\left[\mathbb{A}_{\mathrm{PRF}}^{\$^{\prime}, \ell[\mathcal{F}]_{k}} \rightarrow 1\right]=\operatorname{Pr}\left[\mathbb{A}^{\mathrm{CFB}\left[\$^{\prime}\right], \ell[\mathcal{E}]_{k}, \ell[\mathcal{D}]_{k}} \text { sets bad }\right] \leq \frac{\sigma(\sigma-1)}{2 \cdot|\mathrm{T}|} .
\end{aligned}
$$

The second case is just the traditional birthday bound, since one of the two inputs to any collision event is random: either as the output of $\$^{\prime}$ or as $I$, which is uniformly random because the adversary is ivrespecting. So, using the identical-until-bad argument above with these bounds,

$$
\begin{aligned}
& \Delta_{\mathbb{A}}\left(\begin{array}{l}
\mathrm{CFB}\left[\mathcal{F}_{k}\right], \ell[\mathcal{E}]_{k}, \ell[\mathcal{D}]_{k} \\
{\left[\mathrm{CFB}\left[\mathcal{F}_{k}\right],, \ell[\mathcal{E}]_{k}, \ell[\mathcal{D}]_{k}\right.}
\end{array}\right) \leq \operatorname{Pr}\left[\mathbb{A}^{\mathrm{CFB}\left[\mathcal{F}_{k}\right], \ell[\mathcal{E}]_{k}, \ell[\mathcal{D}]_{k}} \text { sets bad }\right] \\
& \leq\left|\operatorname{Pr}\left[\mathbb{A}_{\mathrm{PRF}}^{\mathcal{F}_{k}, \ell[\mathcal{F}]_{k}} \rightarrow 1\right]-\operatorname{Pr}\left[\mathbb{A}_{\mathrm{PRF}}^{\$^{\prime}, \ell[\mathcal{F}]_{k}} \rightarrow 1\right]\right|+\frac{\sigma(\sigma-1)}{2 \cdot|\mathrm{T}|} \\
& \leq \operatorname{Adv}_{\mathcal{F} ; \mathcal{L}_{\mathrm{F}}}^{\mathrm{PRLF}}\left(\mathbb{A}_{\mathrm{PRF}}\right)+\frac{\sigma(\sigma-1)}{2 \cdot|\mathrm{T}|}
\end{aligned}
$$

The second term we will bound by constructing an adversary $\mathbb{A}_{\text {PRF }}^{\prime}$ against the LPRF security of $\mathcal{F}$. Let $\mathcal{O}_{c}$ denote the first (challenge) oracle that $\mathbb{A}_{\mathrm{PRF}}^{\prime}$ may interact with. $\mathbb{A}_{\mathrm{PRF}}^{\prime}$ runs $\mathbb{A}$, answering all of $\mathbb{A}$ 's challenge queries with $\mathrm{CFB}\left[\mathcal{O}_{c}\right]$, and answers leakage oracle queries by making the appropriate queries to their own $\ell[\mathcal{F}]_{k}$ oracle. When $\mathbb{A}$ terminates, $\mathbb{A}_{\mathrm{PRF}}$ terminates with the same response. The boxed code ensures inputs to $\mathcal{O}_{c}$ are never forwarded to $\ell[\mathcal{F}]_{k}$, and vice versa, meaning $\mathbb{B}$ never makes prohibited queries. Since the two worlds are simulated exactly,

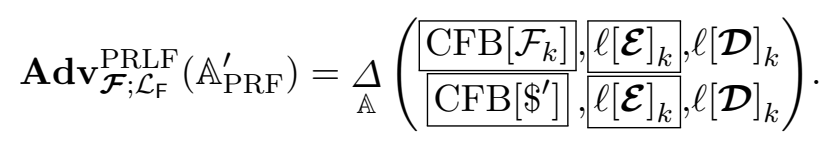

Finally, consider the third term in the expanded bound. Intuitively, this corresponds to distinguishing between $\mathrm{CFB}\left[\$^{\prime}\right]$ and $\$$, except that the bad events are defined across two oracles. As long as $\sigma \leq|\mathrm{T}|$, $\mathrm{CFB}\left[\$^{\prime}\right]$ is a random function: each block of output $\left(C_{i}\right)$ is the xor of an independently sampled uniformly random element $\left(F_{i}\right)$ with an independent input $\left(M_{i}\right)$ and thus independently uniform random. Thus the only difference between the two sets of oracles is whether the adversary can distinguish between $\left(\mathrm{CFB}\left[\$^{\prime}\right],, \ell[\mathcal{E}]_{k}, \ell[\mathcal{D}]_{k}\right)$ and $\left(\left[\mathrm{CFB}\left[\$^{\prime}\right], \ell[\mathcal{E}]_{k}, \ell[\mathcal{D}]_{k}\right)\right.$. Since they are identical-until-bad ${ }_{L}$, this corresponds to a collision between some $C_{i-1}$ during execution of $\ell[\mathcal{E}]_{k}$, with an element of $\mathcal{X}$, a list of uniformly sampled elements. Within $\sigma$ total queries, this is maximised by making $\sigma / 2$ queries to each oracle, and thus

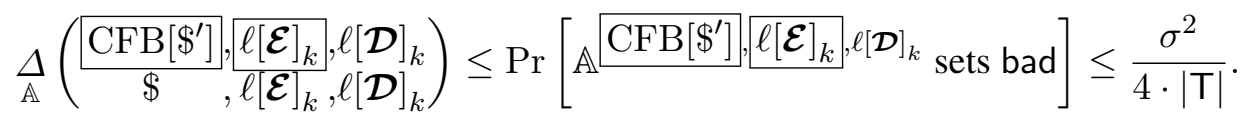

Summing the terms and putting $\mathbb{A}_{P R F}$ to be the adversary that maximises the LPRF advantage (out of $\mathbb{A}_{\mathrm{PRF}}^{\prime}$ and the $\mathbb{A}_{\mathrm{PRF}}$ in this proof) completes the proof. 


\section{6 mrLAE Security by Instantiating the PRF and MAC}

The A5 and SIVAT composition mechanisms can be instantiated with any suitably secure primitives to yield secure nLAE or mrLAE schemes. Together with using $\mathrm{CFB}[\mathcal{F}]$ as underlying iv $\mathcal{E}$, these allow us to construct a secure mrLAE scheme through any PRF $\mathcal{F}$ with a secure implementation $\mathcal{F}$ and a secure MAC implementation $(\mathcal{T}, \mathcal{V})$. The remaining questions therefore are what can be said about securely implementing these primitives and what conclusions for the overall scheme can subsequently be drawn. We will answer these questions from two perspectives: a practical side-channel one (for those favouring masked AES) and a more theoretical, yet eminently implementable one in the continuous leakage model.

A side-channel perspective. Our result provides a roadmap for obtaining a side-channel misuseresistant $\mathrm{AE}$ scheme by selecting reasonable practical primitives (and implementations) for the PRF and the MAC (say a suitably masked AES, respectively KMAC) and subsequently gauging to what extent actual leakage on the primitive implementations can be used to break the relevant PRLF or EUF-CLMA notions as well as whether leakage on the full implementation is cleanly segregated or whether undesired correlation indicates bleeding of leakage from the values or variables from one component into say part of the power trace associated with another component.

The result above no longer explicitly takes into account leakage classes; these have effectively become implicit artefacts of the attack. We assume that a successful attack on the full scheme will be recognized as such: our result essentially says that if such an attack is found then either the leakage is not cleanly separated or one of the primitive implementations is already insecure (or both).

A leakage resilience perspective. A complementary approach to the practical one above is to design the primitives and their implementations with a provable level of resistance against leakage functions from a specific class. As already explained in the introduction, a multitude of models exist depending on the class of functions under consideration. One of the stronger models is that of continuous leakage: here the leakage functions can be arbitrary, subject to the constraint that their range is bounded. A usual refinement is to use a split-state model, where the key's representation $\boldsymbol{k}$ is operated upon in two (or more) tranches and each tranch can only leak on that part of $\boldsymbol{k}$ in scope for the operation at hand (assuming only computation leaks, as usual).

While there are PRFs that have been proven secure in the continuous leakage model, as far as we can tell this has always come at the price of adaptivity. In order for our constructions to be implemented a new PRF is called for, with an implementation secure in the stronger, adaptive continuous leakage model. In Appendix A we provide such a function and implementation, and prove the latter secure in the generic group model (against adaptive continous leakage in a split-state setting). Additionally, we show how to create a related MAC such that leaking on the verification's implementation is ok.

Our construction is an evolution of the MAC of Martin et al. [36], itself inspired by a scheme by Kiltz and Pietrzak [29]. The key enabling novelty is the use of three shares instead of the customary two. A thorough discussion of the design choices, specifications, and security justification can be found in Appendix A but for completeness we provide the final theorem statement below.

Theorem 5. Let SIVAT be the SIVAT mechanism instantiated with the implementations described in Appendix A over a generic group of p elements, and assume that each share of the internal PRF leaks at most $\lambda$ per call following the associated leakage functions, as described by leakage sets $\left(\mathcal{L}_{\text {Enc }}, \mathcal{L}_{\text {Dec }}\right)$. Let $\mathbb{A}$ be an adversary making at most $g$ direct queries to the generic group oracle (including the complexity of all chosen leakage queries) and making q construction queries totalling $\sigma$ blocks. Then,

$$
\mathbf{A d v}_{\mathrm{SIVAT} ; \mathcal{L}_{\mathrm{Enc}}, \mathcal{L}_{\mathrm{Dec}}^{\mathrm{LAE}}}(\mathbb{A}) \leq \frac{7}{p}\left(2^{4 \lambda} \cdot \sigma^{2} \cdot(g+9 q+5 \sigma)^{2}+8(g+9 q+5 \sigma)^{2}\right)
$$

To get a feel for the practical security level, let's look at parameters if the schemes are instantiated over a 512 bit elliptic curves, and we want the keep the attack success probability below $2^{-60}$ (a common 
limit in the real world, e.g. [34]). Let's assume that each internal leakage function leaks at most $\lambda=85$ bits, which is approximately a sixth of a group element. Then the scheme would remain secure until the adversary has encrypted or decrypted around $2^{25}$ blocks, and made a similar number of queries to the generic group.

This result comes with a few caveats, covered in more detail by Appendix A. For instance, to ensure security against the leakage of arbitrary functions of the key, to process $q$ queries of total $\sigma$ blocks the construction must sample $4 q+\sigma$ random group elements in a leakage-resilient manner, which can be complicated [36]. Nonetheless, our construction is proof positive of the existence of leakage resilient authenticated encryption in a very strong sense.

\section{Conclusions and Open Problems}

We introduced notions for strengthened $\mathrm{AE}$ when considering leakage, discussed generic composition under leakage, and showed the EtM type constructions can be proven secure in this context. We give a new scheme, SIVAT, that achieves misuse resistance and leakage resilience simultaneously, and show how this can be bootstrapped from a PRF secure against leakage. Finally, we give a concrete instantiation for the SIVAT mechanism. Our research unveils several interesting open problems, which we summarise subsequently.

IND-aCPLA. If one allows nonce-reuse, then for any leakage set $\mathcal{L}_{\mathrm{E}}$ security against $\mathcal{L}_{\mathrm{E}}$-IND-CPLA adversary implies $\left(\mathcal{L}_{\mathrm{E}}, \mathcal{L}_{\mathrm{E}}{ }^{\prime}\right)$-IND-aCPLA security, where $\mathcal{L}_{\mathrm{E}}{ }^{\prime}$ is the essentially the same set as $\mathcal{L}_{\mathrm{E}}$ with some minor bookkeeping to ensure correct syntax. The implication is trivial as the leakage on any valid $\mathcal{D}$-query can be perfectly simulated by repeating the corresponding $\mathcal{E}$-query instead. In the the nonce or iv respecting cases the implication remains open (as repeating encryption queries including nonce is no longer allowed). Nonetheless, we conjecture that even in these two settings for many reasonable leakage sets $\mathcal{L}_{\mathrm{E}}, \mathcal{L}_{\mathrm{E}}$-IND-CPLA does imply $\left(\mathcal{L}_{\mathrm{E}}, \mathcal{L}_{\mathrm{E}}{ }^{\prime}\right)$-IND-aCPLA. We leave it as an interesting question to formalise this or find a counter-example. More generally, is there some way of defining $\mathcal{L}_{\mathrm{ED}}$ as a function of some general sets $\mathcal{L}_{\mathrm{E}}, \mathcal{L}_{\mathrm{D}}$ such that $\mathcal{L}_{\mathrm{ED}}$-IND-CPLA $\Longrightarrow\left(\mathcal{L}_{\mathrm{E}}, \mathcal{L}_{\mathrm{D}}\right)$-IND-aCPLA?

$M t E$ with restricted leakage sets. The insecurity of the majority of the $\mathrm{MtE}$ schemes when considering leakage comes from a generic attack against any schemes whose inverse follows the decrypt-then-verify or decrypt-and-verify structure. We leave it as an interesting open question to investigate the leakage security under other more restricted leakage sets.

Misuse resistance with minimal message expansion. We demonstrate that misuse resistance can be achieved through generic composition, at the cost of additional message expansion, using a MAC-thenEncrypt-then-MAC structure (leading to SIVAT). We believe that dedicated constructions are likely to exist that can achieve mrLAE security with minimal expansion, or more generally LAE without requiring independent keys.

Acknowledgements. Initial work was conducted while Dan Martin was employed by the Department of Computer Science, University of Bristol. Guy Barwell was supported by an EPSRC grant; Elisabeth Oswald and Dan Martin were in part supported by EPSRC via grants EP/I005226/1 (SILENT) and EP/N011635/1 (LADA). 


\section{References}

1. Alkassar, A., Geraldy, A., Pfitzmann, B., Sadeghi, A.R.: Optimized self-synchronizing mode of operation. In: Matsui, M. (ed.) FSE 2001. LNCS, vol. 2355, pp. 78-91. Springer, Heidelberg (Apr 2002); Cited on page 25.

2. Andreeva, E., Bogdanov, A., Luykx, A., Mennink, B., Mouha, N., Yasuda, K.: How to securely release unverified plaintext in authenticated encryption. In: Sarkar and Iwata [52], pp. 105-125; Cited on pages 3, 4, 5, 6, 15, and 18.

3. Aranha, D.F., Fouque, P.A., Qian, C., Tibouchi, M., Zapalowicz, J.C.: Binary elligator squared. In: Joux, A., Youssef, A.M. (eds.) SAC 2014. LNCS, vol. 8781, pp. 20-37. Springer, Heidelberg (Aug 2014); Cited on page 33.

4. Barwell, G., Page, D., Stam, M.: Rogue decryption failures: Reconciling AE robustness notions. In: Groth [23], pp. 94-111 ; Cited on pages 3, 4, 5, 7, and 15 .

5. Belaïd, S., Fouque, P.A., Gérard, B.: Side-channel analysis of multiplications in GF(2128) - application to AES-GCM. In: Sarkar, P., Iwata, T. (eds.) ASIACRYPT 2014, Part II. LNCS, vol. 8874, pp. 306-325. Springer, Heidelberg (Dec 2014); Cited on page 15 .

6. Bellare, M., Desai, A., Jokipii, E., Rogaway, P.: A concrete security treatment of symmetric encryption. In: 38th FOCS. pp. 394-403. IEEE Computer Society Press (Oct 1997); Cited on page 33.

7. Bellare, M., Kane, D., Rogaway, P.: Big-key symmetric encryption: Resisting key exfiltration. In: Robshaw, M., Katz, J. (eds.) CRYPTO 2016, Part I. LNCS, vol. 9814, pp. 373-402. Springer, Heidelberg (Aug 2016); Cited on page 5.

8. Bellare, M., Namprempre, C.: Authenticated encryption: Relations among notions and analysis of the generic composition paradigm. In: Okamoto, T. (ed.) ASIACRYPT 2000. LNCS, vol. 1976, pp. 531-545. Springer, Heidelberg (Dec 2000) ; Cited on pages 3 and 5 .

9. Bellare, M., Rogaway, P.: Random oracles are practical: A paradigm for designing efficient protocols. In: Ashby, V. (ed.) ACM CCS 93. pp. 62-73. ACM Press (Nov 1993); Cited on pages 32 and 33.

10. Bernstein, D.J.: CAESAR competition call (2013), http://competitions.cr.yp.to/caesar-call-3. html; Cited on pages 5 and 24.

11. Bernstein, D.J., Hamburg, M., Krasnova, A., Lange, T.: Elligator: elliptic-curve points indistinguishable from uniform random strings. In: Sadeghi, A.R., Gligor, V.D., Yung, M. (eds.) ACM CCS 13. pp. 967-980. ACM Press (Nov 2013); Cited on page 33 .

12. Berti, F., Koeune, F., Pereira, O., Peters, T., Standaert, F.X.: Leakage-resilient and misuse-resistant authenticated encryption. Cryptology ePrint Archive, Report 2016/996 (2016), http://eprint.iacr.org/2016/996; Cited on page 5.

13. Boldyreva, A., Degabriele, J.P., Paterson, K.G., Stam, M.: On symmetric encryption with distinguishable decryption failures. In: Moriai, S. (ed.) FSE 2013. LNCS, vol. 8424, pp. 367-390. Springer, Heidelberg (Mar 2014); Cited on pages 3, 4,5 , and 15 .

14. Boldyreva, A., Degabriele, J.P., Paterson, K.G., Stam, M.: Security of symmetric encryption in the presence of ciphertext fragmentation. Cryptology ePrint Archive, Report 2015/059 (2015), http: //eprint.iacr.org/2015/059; Cited on page 3.

15. Boneh, D., Boyen, X., Goh, E.J.: Hierarchical identity based encryption with constant size ciphertext. In: Cramer, R. (ed.) EUROCRYPT 2005. LNCS, vol. 3494, pp. 440-456. Springer, Heidelberg (May 2005); Cited on page 33.

16. Cohn-Gordon, K., Cremers, C., Dowling, B., Garratt, L., Stebila, D.: A formal security analysis of the signal messaging protocol. Cryptology ePrint Archive, Report 2016/1013 (2016), http: //eprint.iacr.org/2016/1013; Cited on page 5.

17. Dodis, Y., Kalai, Y.T., Lovett, S.: On cryptography with auxiliary input. In: Mitzenmacher, M. (ed.) 41st ACM STOC. pp. 621-630. ACM Press (May / Jun 2009); Cited on pages 3, 4, and 15.

18. Dodis, Y., Pietrzak, K.: Leakage-resilient pseudorandom functions and side-channel attacks on Feistel networks. In: Rabin, T. (ed.) CRYPTO 2010. LNCS, vol. 6223, pp. 21-40. Springer, Heidelberg (Aug 2010); Cited on pages 6, 13, and 33.

19. Dziembowski, S., Pietrzak, K.: Leakage-resilient cryptography. In: 49th FOCS. pp. 293-302. IEEE Computer Society Press (Oct 2008); Cited on pages 3, 4, and 6.

20. Faust, S., Pietrzak, K., Schipper, J.: Practical leakage-resilient symmetric cryptography. In: Prouff, E., Schaumont, P. (eds.) CHES 2012. LNCS, vol. 7428, pp. 213-232. Springer, Heidelberg (Sep 2012); Cited on pages 6, 13, and 33.

21. Fischlin, M., Günther, F., Marson, G.A., Paterson, K.G.: Data is a stream: Security of stream-based channels. In: Gennaro, R., Robshaw, M.J.B. (eds.) CRYPTO 2015, Part II. LNCS, vol. 9216, pp. 545-564. Springer, Heidelberg (Aug 2015) ; Cited on page 3 .

22. Galindo, D., Vivek, S.: A practical leakage-resilient signature scheme in the generic group model. In: Knudsen, L.R., Wu, H. (eds.) SAC 2012. LNCS, vol. 7707, pp. 50-65. Springer, Heidelberg (Aug 2013); Cited on page 32.

23. Groth, J. (ed.): 15th IMA International Conference on Cryptography and Coding, LNCS, vol. 9496. Springer, Heidelberg (Dec 2015); Cited on pages 30 and 31.

24. Hazay, C., López-Alt, A., Wee, H., Wichs, D.: Leakage-resilient cryptography from minimal assumptions. In: Johansson, T., Nguyen, P.Q. (eds.) EUROCRYPT 2013. LNCS, vol. 7881, pp. 160-176. Springer, Heidelberg (May 2013); Cited on pages 5 and 15 .

25. Hoang, V.T., Krovetz, T., Rogaway, P.: Robust authenticated-encryption AEZ and the problem that it solves. In: Oswald, E., Fischlin, M. (eds.) EUROCRYPT 2015, Part I. LNCS, vol. 9056, pp. 15-44. Springer, Heidelberg (Apr 2015); Cited on pages $3,5,19$, and 24 .

26. Ishai, Y., Sahai, A., Wagner, D.: Private circuits: Securing hardware against probing attacks. In: Boneh, D. (ed.) CRYPTO 2003. LNCS, vol. 2729, pp. 463-481. Springer, Heidelberg (Aug 2003); Cited on pages 4 and 15. 
27. Katz, J., Lindell, Y.: Introduction to Modern Cryptography. Chapman \& Hall/CRC (2008); Cited on page 6.

28. Katz, J., Vaikuntanathan, V.: Signature schemes with bounded leakage resilience. In: Matsui, M. (ed.) ASIACRYPT 2009. LNCS, vol. 5912, pp. 703-720. Springer, Heidelberg (Dec 2009); Cited on page 15.

29. Kiltz, E., Pietrzak, K.: Leakage resilient ElGamal encryption. In: Abe, M. (ed.) ASIACRYPT 2010. LNCS, vol. 6477, pp. 595-612. Springer, Heidelberg (Dec 2010); Cited on pages 3, 4, 28, and 32.

30. Kocher, P.C.: Timing attacks on implementations of Diffie-Hellman, RSA, DSS, and other systems. In: Koblitz, N. (ed.) CRYPTO’96. LNCS, vol. 1109, pp. 104-113. Springer, Heidelberg (Aug 1996); Cited on page 3.

31. Kocher, P.C., Jaffe, J., Jun, B.: Differential power analysis. In: Wiener, M.J. (ed.) CRYPTO'99. LNCS, vol. 1666, pp. 388-397. Springer, Heidelberg (Aug 1999); Cited on page 3.

32. Kurosawa, K., Phong, L.T.: Leakage resilient IBE and IPE under the DLIN assumption. In: Jacobson Jr., M.J., Locasto, M.E., Mohassel, P., Safavi-Naini, R. (eds.) ACNS 13. LNCS, vol. 7954, pp. 487-501. Springer, Heidelberg (Jun 2013); Cited on page 15 .

33. Longo, J., Martin, D.P., Oswald, E., Page, D., Stam, M., Tunstall, M.: Simulatable leakage: Analysis, pitfalls, and new constructions. In: Sarkar and Iwata [52], pp. 223-242; Cited on page 5.

34. Luykx, A., Paterson, K.: Limits on authenticated encryption use in tls (2016), http://www.isg.rhul.ac.uk/ $\sim \mathrm{kp} / \mathrm{TLS}-\mathrm{AEb}$ ound.s.pdf; Cited on page 29.

35. Mangard, S., Oswald, E., Popp, T.: Power Analysis Attacks: Revealing the Secrets of Smart Cards. Springer (2008); Cited on page 15.

36. Martin, D.P., Oswald, E., Stam, M., Wójcik, M.: A leakage resilient MAC. In: Groth [23], pp. 295-310; Cited on pages 4, $6,14,28,29,32,33$, and 41.

37. Maurer, U.M.: Abstract models of computation in cryptography (invited paper). In: Smart, N.P. (ed.) 10th IMA International Conference on Cryptography and Coding. LNCS, vol. 3796, pp. 1-12. Springer, Heidelberg (Dec 2005); Cited on page 33.

38. McGrew, D.A., Viega, J.: The security and performance of the galois/counter mode of operation (full version). Cryptology ePrint Archive, Report 2004/193 (2004), http: / /eprint.iacr.org/2004/193; Cited on page 15.

39. Micali, S., Reyzin, L.: Physically observable cryptography (extended abstract). In: Naor, M. (ed.) TCC 2004. LNCS, vol. 2951, pp. 278-296. Springer, Heidelberg (Feb 2004); Cited on pages 3, 4, 15, and 18.

40. Namprempre, C., Rogaway, P., Shrimpton, T.: Reconsidering generic composition. In: Nguyen, P.Q., Oswald, E. (eds.) EUROCRYPT 2014. LNCS, vol. 8441, pp. 257-274. Springer, Heidelberg (May 2014); Cited on pages 3, 4, 5, and 9.

41. Nechaev, V.I.: Complexity of a determinate algorithm for the discrete logarithm. Mathematical Notes 55(2), 165-172 (1994); Cited on page 33.

42. NIST: FIPS 81: DES Modes of Operation. Issued December 2, 63 (1980); Cited on page 3.

43. Pereira, O., Standaert, F.X., Vivek, S.: Leakage-resilient authentication and encryption from symmetric cryptographic primitives. In: Ray, I., Li, N., Kruegel:, C. (eds.) ACM CCS 15. pp. 96-108. ACM Press (Oct 2015); Cited on page 5.

44. Perrin, T.: Double ratchet algorithm (2014), https://github.com/trevp/double_ratchet/wiki, Retrieved 2016-09-01; Cited on page 5.

45. Pietrzak, K.: A leakage-resilient mode of operation. In: Joux, A. (ed.) EUROCRYPT 2009. LNCS, vol. 5479, pp. $462-482$. Springer, Heidelberg (Apr 2009); Cited on page 6.

46. Qin, B., Liu, S.: Leakage-flexible CCA-secure public-key encryption: Simple construction and free of pairing. In: Krawczyk, H. (ed.) PKC 2014. LNCS, vol. 8383, pp. 19-36. Springer, Heidelberg (Mar 2014); Cited on page 5.

47. Renauld, M., Standaert, F.X., Veyrat-Charvillon, N., Kamel, D., Flandre, D.: A formal study of power variability issues and side-channel attacks for nanoscale devices. In: Paterson, K.G. (ed.) EUROCRYPT 2011. LNCS, vol. 6632, pp. 109-128. Springer, Heidelberg (May 2011); Cited on page 18.

48. Rogaway, P.: Authenticated-encryption with associated-data. In: Atluri, V. (ed.) ACM CCS 02. pp. 98-107. ACM Press (Nov 2002); Cited on page 6.

49. Rogaway, P.: Nonce-based symmetric encryption. In: Roy, B.K., Meier, W. (eds.) FSE 2004. LNCS, vol. 3017, pp. 348359. Springer, Heidelberg (Feb 2004); Cited on page 6.

50. Rogaway, P., Bellare, M., Black, J., Krovetz, T.: OCB: A block-cipher mode of operation for efficient authenticated encryption. In: ACM CCS 01. pp. 196-205. ACM Press (Nov 2001); Cited on page 6.

51. Rogaway, P., Shrimpton, T.: A provable-security treatment of the key-wrap problem. In: Vaudenay, S. (ed.) EUROCRYPT 2006. LNCS, vol. 4004, pp. 373-390. Springer, Heidelberg (May / Jun 2006); Cited on pages 3, 5, and 24.

52. Sarkar, P., Iwata, T. (eds.): ASIACRYPT 2014, Part I, LNCS, vol. 8873. Springer, Heidelberg (Dec 2014); Cited on pages 30 and 31 .

53. Schipper, J.: Leakage-Resilient Authentication. Ph.D. thesis, Utrecht University (2010); Cited on page 5.

54. Shoup, V.: Lower bounds for discrete logarithms and related problems. In: Fumy, W. (ed.) EUROCRYPT'97. LNCS, vol. 1233, pp. 256-266. Springer, Heidelberg (May 1997); Cited on pages 33 and 34.

55. Shrimpton, T., Terashima, R.S.: A modular framework for building variable-input-length tweakable ciphers. In: Sako, K., Sarkar, P. (eds.) ASIACRYPT 2013, Part I. LNCS, vol. 8269, pp. 405-423. Springer, Heidelberg (Dec 2013) ; Cited on pages 19 and 43.

56. Standaert, F.X., Pereira, O., Yu, Y.: Leakage-resilient symmetric cryptography under empirically verifiable assumptions. In: Canetti, R., Garay, J.A. (eds.) CRYPTO 2013, Part I. LNCS, vol. 8042, pp. 335-352. Springer, Heidelberg (Aug 2013) ; Cited on pages 3, 4, and 15.

57. Yau, A.K.L., Paterson, K.G., Mitchell, C.J.: Padding oracle attacks on CBC-mode encryption with secret and random IVs. In: Gilbert, H., Handschuh, H. (eds.) FSE 2005. LNCS, vol. 3557, pp. 299-319. Springer, Heidelberg (Feb 2005); Cited on page 3 . 
58. Yu, Y., Standaert, F.X., Pereira, O., Yung, M.: Practical leakage-resilient pseudorandom generators. In: Al-Shaer, E., Keromytis, A.D., Shmatikov, V. (eds.) ACM CCS 10. pp. 141-151. ACM Press (Oct 2010); Cited on page 15.

\section{A A PRF and MAC Resilient Against Continuous and Fully Adaptive Leakage}

In this section we describe how one can instantiate our generic scheme with concrete functions, again within the OCLI paradigm. At the lowest level, we construct LPRF and LMAC implementations where each algorithm consists of three phases. These implementations are secure as long as the phases leak independently, and no phase leaks more than $\lambda$ bits.

We begin by restating the MAC of Martin et al. [36], on which our constructions are based. We prove that using three shares, the tagging implementation is a LPRF (but two shares are not sufficient). Next, we enhance the MAC by adjusting the verification routine such that it remains secure under leakage. Finally we consider the leakage resilience of $A 5$ and SIVAT when instantiated with these primitives and CFB mode.

\section{A.1 Recalling the MOSW MAC}

Martin et al. [36] designed a MAC that computes the pairing of the key with a hash of the message. Using a technique of Kiltz and Pietrzak [29], they demonstrate how to split the tagging oracle into two shares. We will refer to it as the MOSW construction and this tagging implementation as $\mathcal{T}_{2}$. It has been reproduced in Figure 12, using slightly different notation to the original (see below).

They prove it secure against adaptive leakage on tagging queries from the leakage set $\mathcal{L}_{\mathrm{T}_{2}}$, the set containing all functions of the form

$$
L_{T_{2}}\left(\left(S^{\ominus}, S^{\ominus}\right), M ; r\right)=\left(L^{\ominus}\left(S^{\ominus}, M ; r\right), L^{\ominus}\left(S^{\ominus}, H(M), Y^{\ominus} ; r\right)\right)
$$

where $L^{\ominus}$ and $L^{\ominus}$ output $\lambda$ bits, $S^{\ominus}$ and $S^{\ominus}$ is the secret information used by each half, $r$ is the randomness, and $Y^{\ominus}$ is the information passed from the first half of the function to the second half. It will form the basis for our constructions, but is not itself suitable for any of our requirements, since the authors do not provide an implementation secure under verification leakage, nor is it a PRLF (in the next section we will show that it is a PRF, but not a PRLF).

A note on notation. The symbols $\ominus, \ominus$ and later $\ominus$ will be used to refer to the different shares and subroutines used within the function. They are run sequentially from "top to bottom".

In the MOSW work, $\mathcal{T}_{2}^{\ominus}$ generated the randomness it required and passed it to $\mathcal{T}_{2}^{\ominus}$. Here, we have $\mathcal{T}_{2}$ generate all randomness and pass it into the functions. This is to match our definition of an implementation and keep the functions themselves more concise. However, as discussed in the MOSW work, how randomness is generated effects the amount of information that an adversary can extract from a device, and thus it must still be taken into consideration. Instead of giving the leakage function access to $W$, we write $H(M)$, to show how the input to the leakage function relates to the inputs of the functions.

\section{A.2 Modelling Discussion}

Before giving the details of the primitives used to instantiate the generic constructions from Section 5, we describe any assumptions that will be required for the remainder of this work. We explicitly detail any subtleties that arise from the interaction of leakage and well studied models.

Model assumptions. In the leakage free case, our constructions are secure in the random oracle model [9]. When leakage is available, we will extend to the generic group model, allowing us to model internal curve points. This is in contrast to the other works in the literature [22,29] that require the generic group model to achieve security even without leakage. 


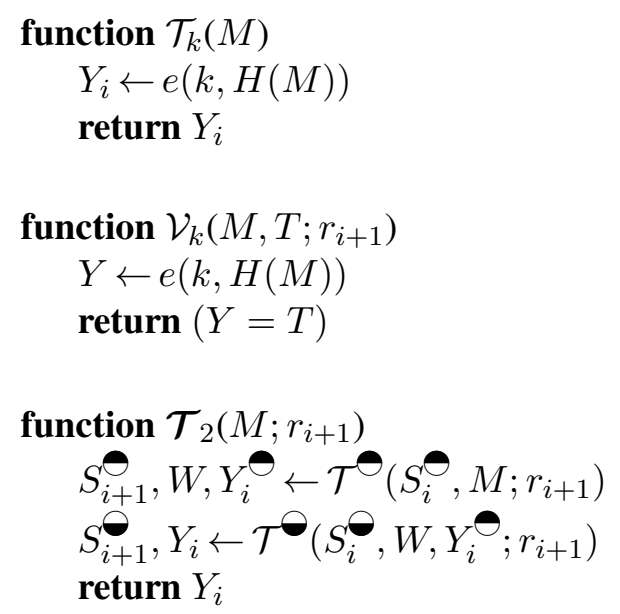

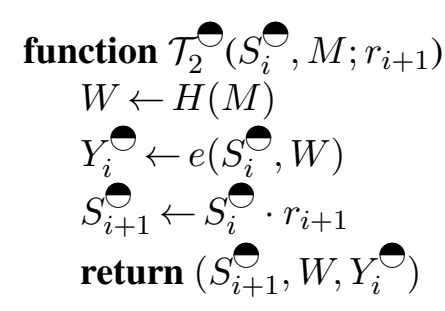

function $\mathcal{T}_{2}^{\ominus}\left(S_{i}^{\ominus}, W, Y_{i}^{\ominus} ; r_{i+1}\right)$

$Y_{i}^{\ominus} \leftarrow e\left(S_{i}^{\ominus}, W\right)$

$Y_{i} \leftarrow Y_{i}^{\ominus} \cdot Y_{i}^{\ominus}$

$S_{i+1}^{\ominus} \leftarrow S_{i}^{\ominus} \cdot r_{i+1}^{-1}$

return $\left(S_{i+1}^{\ominus}, Y_{i}\right)$

Fig. 12: The MOSW MAC Construction [36]. On the left are the tagging and verification functions $\mathcal{T}_{k}$ and $\mathcal{V}_{k}$. Below them is $\mathcal{T}_{2}$, a secure $\left(\mathcal{L}_{\mathrm{T}_{2}}, \emptyset\right)$-LMAC implementation of the tagging function. The key and computation are split into two shares shown on the right, which can be assumed to leaks independently.

The generic group model $[37,41,54]$ was designed to model groups where the adversary cannot exploit the structure of the underlying group. The model we follow here, due to Shoup [54], achieves this by representing group elements as random bit strings. The bilinaer GGM [15] extends this to provision a bilinear pairing $e$ between groups, acting in a similar way. The only operation an adversary can perform locally is equality testing (by comparison of bitstrings), while to perform the group operation or bilinear pairing they must interact with their oracles. A result of using the GGM to model a group, it implicity assumes that the group operations themselves do not leak. Therefore, in practice, the group operations would have to be implemented using counter-measures.

We also assume the existence of a secure hash function, which we model in the random oracle model [9]. The random oracle is publicly accessible, and so we provide both the adversary and their leakage functions implicit access to it. We need not consider additional leakage from random oracle queries, since in every use case their inputs will already be known to the adversary or the leakage function.

Caveats and limitations. Our proofs also assume evaluation of an arbitrary sized input hash function takes a fixed amount of effort. If the hash did not support this functionality, the advantage of our two general constructions would be slightly different as the PRLF and MAC hash more data in SIVAT than in A5, although under valid decryption queries, SIVAT actually hashes less data.

Another caveat to our results is that this particular instantiation provides indistinguishability from a stream of random elliptic curve group elements, and not indistinguishability from random bit strings. While this is a slightly weaker notion of security, it still implies the key requirements of traditional leftor-right, real-or-random security [6]. Work by a number of authors on "Elligators" [3,11] has investigated how to efficiently convert pseudo-random elliptic curve points into pseudo-random bitstrings, but are not without issue.

\section{A.3 A Leakage Resilient PRF}

Since at the base level a PRLF instantiates the majority of our components for the generic composition, we begin by constructing this. There have been two leakage resilient PRFs in the continuous leakage model $[18,20]$, but neither provides adaptive security. We claim that $\mathcal{T}$ is a secure PRF in the classical setting, and can be implemented as a PRLF. Thus we begin by showing that the output is indeed pseudo random in the leakage-free case (Theorem 7). The proof is extremely similar to that for the original MAC, except the reduction goes to a decisional bilinear Diffie-Hellman assumption, rather than the computational version used by Martin et al. [36]. 

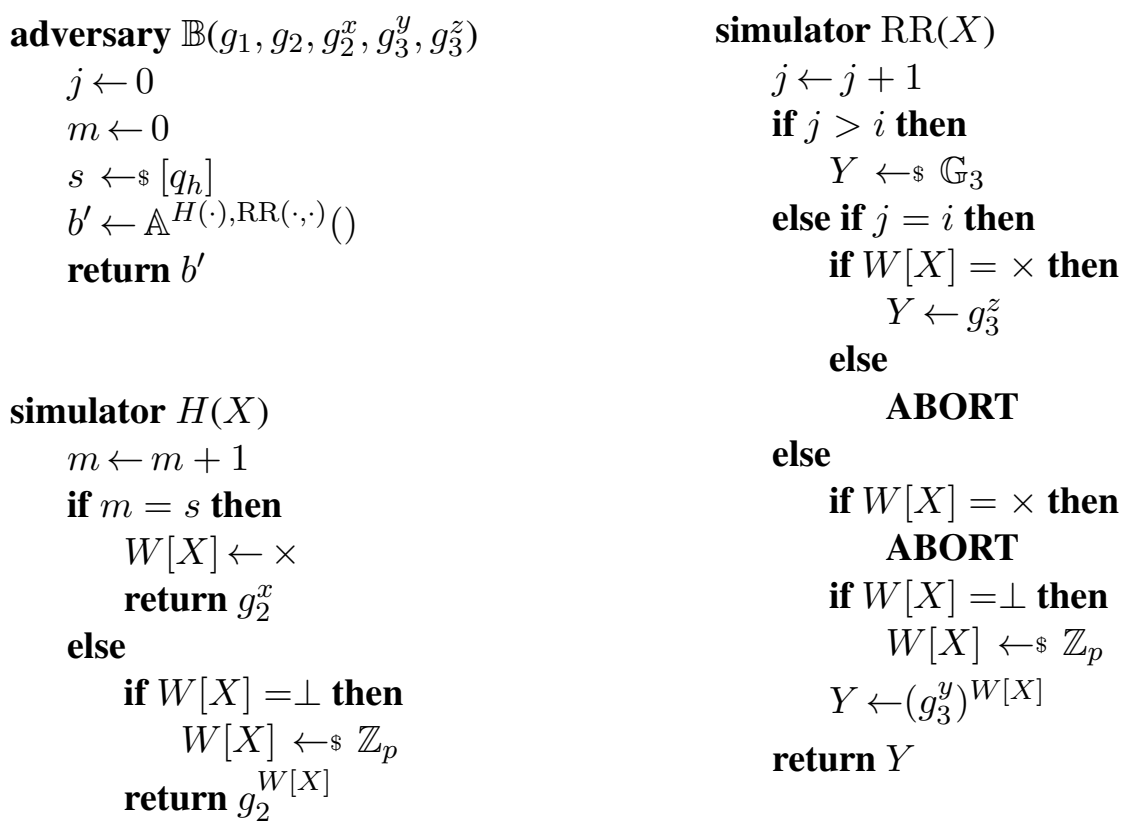

ABORT

else

if $W[X]=\times$ then

ABORT

if $W[X]=\perp$ then $W[X] \leftarrow \$ \mathbb{Z}_{p}$ $Y \leftarrow\left(g_{3}^{y}\right)^{W[X]}$

return $Y$

Fig. 13: Adversary $\mathbb{B}$ simulates the hybrid games $H_{i-1}, H_{i}$ for adversary $\mathbb{A}$

Definition 13 (Decisional Target Bilinear Diffie-Hellman Problem (DTBDH)). For some group size $p$, let $\mathbb{B} \mathbb{G}=\left(\mathbb{G}_{1}, \mathbb{G}_{2}, \mathbb{G}_{3}, e, p\right)$ be a collection of groups with a pairing $e: \mathbb{G}_{1} \times \mathbb{G}_{2} \rightarrow \mathbb{G}_{3}$ between them. The decisional target bilinear Diffie-Hellman problem is then defined as: given $g_{1}, g_{2}, g_{2}^{x}, g_{3}^{y}, g_{3}^{z}$ determine whether $g_{3}^{z}=g_{3}^{x \cdot y}$ or $g_{3}^{z}=g_{3}^{r}$, where $x, y, r$ are chosen uniformly at random from $\mathbb{Z}_{p}$ and $z=x \cdot y$ if $b=1$ and $r$ otherwise for $b$ chosen uniformly at random from $\{0,1\}$. The advantage of an adversary $\mathbb{A}$ is defined as:

$$
\operatorname{Adv}_{\mathbb{B} \mathbb{G}}^{\mathrm{DTBDH}}(\mathbb{A}):=\operatorname{Pr}\left[\mathbb{A}\left(g_{1}, g_{2}, g_{2}^{x}, g_{3}^{y}, g_{3}^{x y}\right)=1\right]-\operatorname{Pr}\left[\mathbb{A}\left(g_{1}, g_{2}, g_{2}^{x}, g_{3}^{y}, g_{3}^{r}\right)=1\right] .
$$

Reassuringly, in the generic group this problem is hard, with the adversarial advantage essentially limited to a birthday-bound style collision attack (the proof is similar to [54]).

Theorem 6. Let the bilinear groups $\mathbb{B} \mathbb{G}$, of size p, be generic groups. Then there exists an adversary $\mathbb{A}$ against the DTBDH game such that:

$$
\mathbf{A d v}_{\mathbb{B} \mathbb{G}}^{\mathrm{DTBDH}}(\mathbb{A}) \leq \frac{\gamma(\gamma-1)}{2 \cdot p}
$$

where $\gamma$ is the number of generic group elements constructed during the game.

We now derive a PRF security bound for a the MOSW tagging function, based on this hard problem.

Theorem 7. Let $\mathbb{A}$ be an adversary against the query PRF security of $\mathcal{T}: \mathbb{G}_{1} \times \mathbb{G}_{2} \rightarrow \mathbb{G}_{3}$. Then there exists an adversary $\mathbb{B}$ (of similar complexity to $\mathbb{A}$ ) who can break the DTBDH assumption for $\mathbb{B} \mathbb{G}$, such that

$$
\operatorname{Adv}_{\mathcal{T}}^{\mathrm{PRF}}(\mathbb{A}) \leq q_{h} \cdot q_{r} \cdot \mathbf{A d v}_{\mathbb{B} \mathbb{G}}^{\mathrm{DTBDH}}(\mathbb{B}),
$$

where $q_{h}$ is the number of queries made to the random oracle and $q_{r}$ is the number of queries to the challenge oracle.

Proof. Let $\mathbb{A}$ be an adversary against the query PRF security of $\mathcal{T}$ (assume without loss of generality the adversary makes unique queries), then it is possible to construct a series of hybrid games (given in Fig. 13). In game $H_{i}$ the first $i$ queries correspond to real queries, while the remaining queries are 


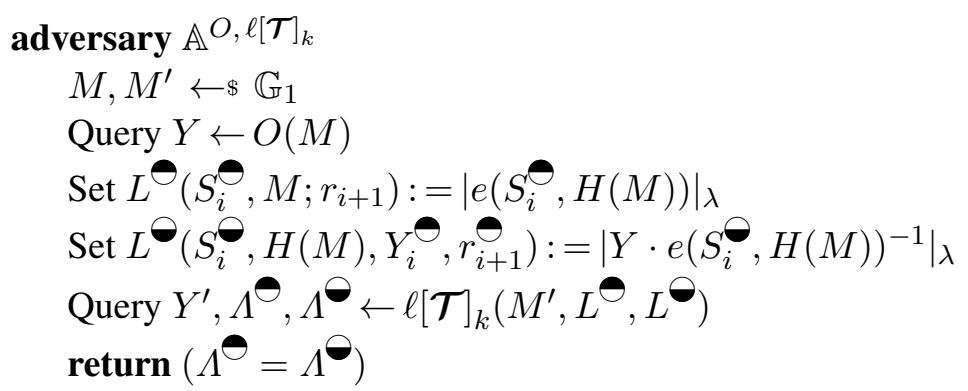

Fig. 14: An adversary $\mathbb{A}$ against the $\mathcal{L}_{\mathrm{T}_{2}}-\mathrm{PRLF}$ security of $\mathcal{T}$. With two generic group elements and two oracle queries the adversary distinguishes whether $O$ implements $\mathcal{T}$ or is ideal with probability $1-2^{-\lambda}$.

responded to with random queries. Game $H_{q_{r}}$ corresponds to the real world while $H_{0}$ corresponds to the random world. If $\mathbb{A}$ can distinguish between these two games with probability $\epsilon$ there exists two consecutive games $H_{i-1}, H_{i}$ that they can distinguish between, with probability at least $\frac{\epsilon}{q_{r}}$. Figure 13 shows how an adversary $\mathbb{B}$ against the DTBDH assumption can simulate these two games for adversary $\mathbb{A}$.

Assume without loss of generality that the value $X$ sent to the PRF on the $i^{\text {th }}$ query was sent to the random oracle beforehand. If $\mathbb{B}$ can guess which query to the random oracle the value $X$ was sent, the reduction will behave as expected. As the oracle will return $g_{3}^{z}$, which is either $g_{3}^{x y}$ or $g_{3}^{r}$ corresponding to the two hybrids $H_{i}$ or $H_{i-1}$. The probability that this happens is $\frac{1}{q_{h}}$.

When the adversary $\mathbb{B}$ can guess which oracle query the $i^{\text {th }}$ challenge query is, all queries $j>i$ return random results, while if $j<i$ the RR oracle returns real values (it will not trigger the abort since the $X$ which has the flag set is query $i$ ). Query $i$ in this scenario returns the DTBDH challenge. It then follows that $z$ is real or random depending if the adversary is playing hybrid $H_{i}$ or $H_{i-1}$ respectively. Thus, if adversary $\mathbb{A}$ can distinguish between these two hybrid games, $\mathbb{B}$ can win the DTBDH game. Putting it all together gives the desired result.

PRF and LMAC does not imply LPRF. Given the traditional similarities between a MAC and a PRF, it would be reasonable to expect that, given that $\mathcal{T}$ is both a PRF and $\left(\mathcal{L}_{\mathrm{T}_{2}}, \emptyset\right)$-LMAC secure, it might also be $\mathcal{L}_{\mathrm{T}_{2}}$-PRLF. However, this is not the case, once again highlighting the surprising effects of allowing adaptive leakage. Figure 14 defines an adversary that can distinguish between a real and random challenge (even in the GGM) when the leakage set is all leakage functions which output $\lambda$ bits from each half of the computation (while obeying the OCLI assumption).

When the challenge is a real PRF call, $Y=e(k, H(M))$ and so $\Lambda_{i}^{\ominus}$ is $\lambda$ bits of $e\left(S_{i}^{\ominus}, H(M)\right)$ and $\Lambda_{i}^{\ominus}$ is $\lambda$ bits of $Y \cdot e\left(S_{i}^{\ominus}, H(M)\right)^{-1}=e\left(S_{i}^{\ominus}, H(M)\right)$ and therefore the two leakage results are equal with probability one. However, when $Y$ is from $\$, \Lambda_{i}^{\ominus}$ will provide $\lambda$ bits of a random element and therefore the two outputs are the same with probability $2^{-\lambda}$. Hence the adversary can win the PRLF game with probability $1-2^{-\lambda}$.

Constructing a secure PRLF. While it is not possible to show PRLF security for an implementation with two sections, we are able to recover security with just one further share, forming the three share implementation shown in Figure 15. The proof reduces from the leakage setting to the leak-free case, which we already proved secure. Following the OCLI assumption, it is proven secure for leakage set $\mathcal{L}_{\mathrm{T}}$ containing all functions of the form:

$$
\begin{aligned}
& L_{T}\left(\left(S^{\ominus}, S^{\ominus}, S^{\ominus}\right), M ;\left(r^{\ominus}, r^{\ominus}\right)\right) \\
& \quad=\left(L^{\ominus}\left(S^{\ominus}, M ; r^{\ominus}\right), L^{\ominus}\left(S^{\ominus}, H(M) ; r^{\ominus}\right), L^{\ominus}\left(S^{\ominus}, H(M), Y^{\ominus}, Y^{\ominus} ; r^{\ominus}, r^{\ominus}\right)\right),
\end{aligned}
$$



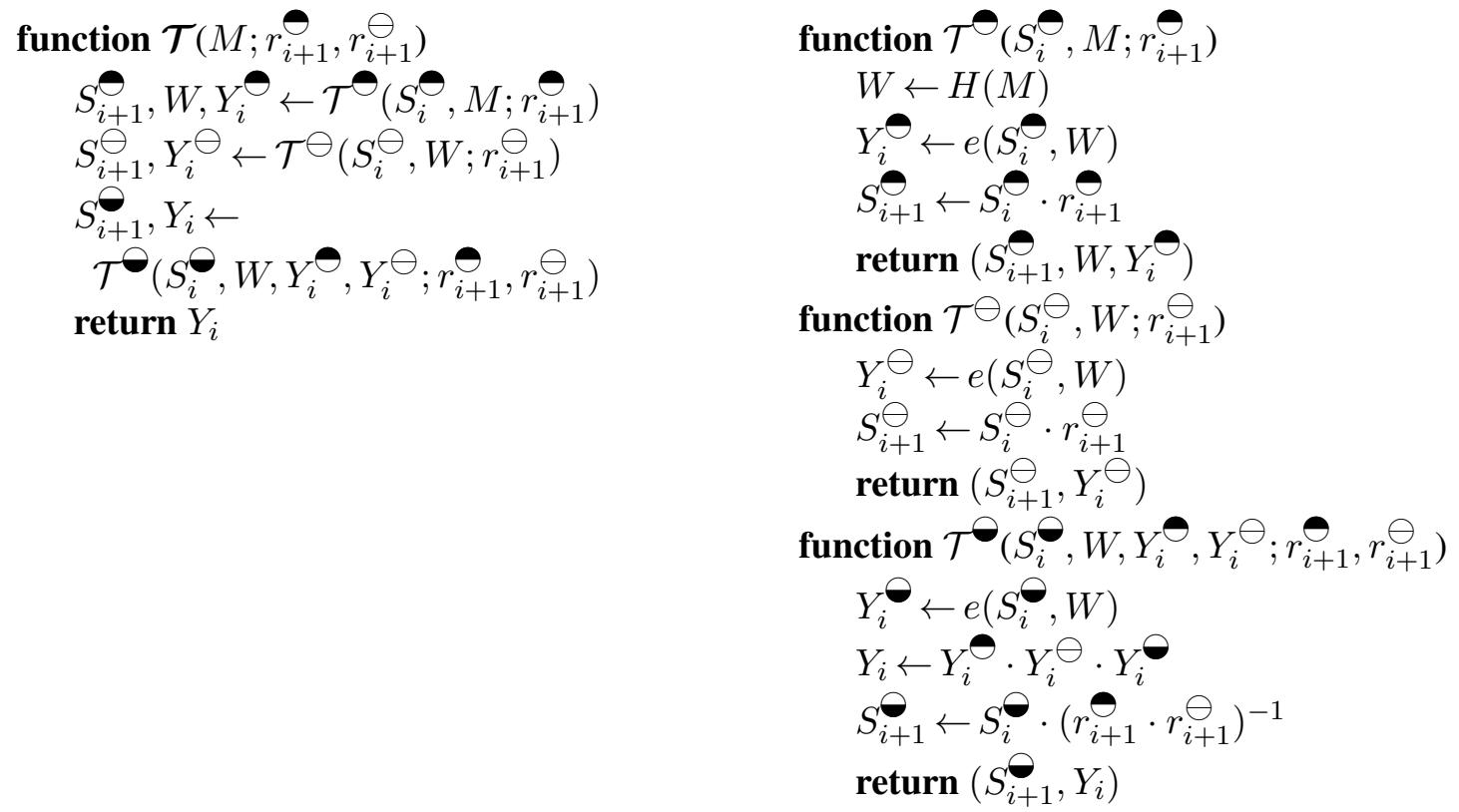

Fig. 15: A secure PRLF implementation $\mathcal{T}$ using three shares.

where $L^{\ominus}, L^{\ominus}$ and $L^{\ominus}$ output $\lambda$ bits, $S^{\ominus}, S^{\ominus}$ and $S^{\ominus}$ is the secret information used by each part, $r^{\ominus}, r^{\ominus}$ is the randomness, and $Y^{\ominus}, Y^{\ominus}$ is the information passed from the first two parts of the function to the final part.

Theorem 8. Let $\mathbb{A}$ be an adversary against the $\mathcal{L}_{\mathrm{T}-P R L F}$ security of $\mathcal{T}$ in the generic group model, then there exists an adversary $\mathbb{A}_{\mathrm{PRF}}$ (of similar complexity to $\mathbb{A}$ ) against the PRF security of $\mathcal{T}$ such that

$$
\operatorname{Adv}_{\mathcal{T}_{i} ; \mathcal{L}_{T}}^{\mathrm{PRLF}}(\mathbb{A}) \leq 2^{4 \cdot \lambda} \cdot \mathbf{A d v}_{\mathcal{T}}^{\mathrm{PRF}}\left(\mathbb{A}_{\mathrm{PRF}}\right)+\frac{\gamma(\gamma-1)}{p}
$$

where $\gamma$ is the number generic group elements, $p$ is the size of the group and $\lambda$ is the amount of leakage allowed per share of the PRF, giving $3 \cdot \lambda$ bits leakage per function call.

Proof. The proof is given in the Generic Group Model and shows that the use of leakage does not allow the adversary to learn any elements that they would be unable to learn if no leakage had been involved. Once this has been shown, it follows that the adversary's advantage can at most be increased by the number of bits that can be learnt about a single element. By showing that each element is only leaked, at most, four times, the adversary's advantage can, at most, be increased by $2^{4 \cdot \lambda}$ over the advantage in the game where no leakage is involved.

Group elements will be represented by polynomials, which will be instantiated at the end of the computation. The polynomials allow the game to keep track of which elements the adversary has asked for in a straightforward manner and because they are instantiated at the end of the computation, the adversary's decisions clearly cannot depend on the actual values of the elements. It must be shown that the chance of these polynomials colliding when evaluated is small (it will be shown to be $\frac{\gamma(\gamma-1)}{2 \cdot p}$ ). Since the PRLF involves three groups, polynomials will be tracked per group.

Let $\mathcal{K},\left\{\mathcal{R}_{j}^{\ominus}\right\}_{j=0}^{q_{R}}\left\{\mathcal{R}_{j}^{\ominus}\right\}_{j=0}^{q_{R}},\left\{\mathcal{H}_{i}\right\}_{j=1}^{q_{H}},\left\{\mathcal{U}_{j}\right\}_{j=1}^{2 \cdot q_{O}},\left\{\mathcal{V}_{j}\right\}_{j=1}^{2 \cdot q_{O}},\left\{\mathcal{W}_{j}\right\}_{j=1}^{2 \cdot q_{O}},\left\{\mathcal{Y}_{j}\right\}_{j=0}^{q_{R}}$ be indeterminants where $q_{H}$ is the number of hash queries, $q_{R}$ is the number of calls to the challenge oracle, $q_{F}$ is the number of calls made to the PRF oracle and $q_{O}$ is the number of group oracle calls (by the adversary). The indeterminants represent the following; $\mathcal{K}$ is the secret key, $\left\{\mathcal{R}_{j}^{\ominus}\right\}_{j=0}^{q_{R}},\left\{\mathcal{R}_{j}^{\ominus}\right\}_{j=0}^{q_{R}}$ are the randomness used to update the key, $\left\{y_{j}\right\}_{j=0}^{q_{R}}$ is the output from the challenge oracle, $\left\{\mathcal{H}_{i}\right\}_{j=1}^{q_{H}}$ represent any hash function queries and $\left\{\mathcal{U}_{j}\right\}_{j=1}^{2 \cdot q_{O}},\left\{\mathcal{V}_{j}\right\}_{j=1}^{2 \cdot q_{O}},\left\{\mathcal{W}_{j}\right\}_{j=1}^{2 \cdot q_{O}}$ represent any elements that are guessed in $\mathbb{G}_{1}, \mathbb{G}_{2}, \mathbb{G}_{3}$ respectively. Let $q=q_{H}+8 \cdot q_{R}+8 \cdot q_{F}+3 \cdot q_{O}$, the factor 3 arises from the fact the adversary can guess the representation of two elements being passed into a binary operation and learns another from 
the output, thus adding, at most, 3 elements to the list per oracle call. Calling either the challenge or PRF oracle adds 8 polynomials to the list; the 3 updated shares, the PRF output and an extra element (since the calculuation requires two multiplications) and the three intermediate values $Y^{\ominus}, Y^{\ominus}, Y^{\ominus}$. The lists $\mathcal{L}_{1}, \mathcal{L}_{2}, \mathcal{L}_{3}$ are used to keep track of polynomials and their representations in $\mathbb{G}_{1}, \mathbb{G}_{2}, \mathbb{G}_{3}$ respectively. They are initialised as follows:

$$
\begin{aligned}
& \mathcal{L}_{1}=\left\{\left(1, \xi_{1}^{1}\right)\right\} \cup\left\{\left(\mathcal{R}_{j}^{\ominus}, \xi_{i+2}^{1}\right)\right\}_{j=0}^{q_{R}} \cup\left\{\left(\mathcal{R}_{j}^{\ominus}, \xi_{i+q_{R}+2}^{1}\right)\right\}_{j=0}^{q_{T}} \\
& \mathcal{L}_{2}=\left\{\left(1, \xi_{1}^{2}\right)\right\} \\
& \mathcal{L}_{3}=\left\{\left(1, \xi_{1}^{3}\right)\right\}
\end{aligned}
$$

where the $\xi_{j}^{i}$ are chosen uniformly at random from $\Xi^{i}$, such that all polynomials have a unique representation. The sets the representations are drawn from $\Xi^{1}, \Xi^{2}, \Xi^{3}$ (for $\mathbb{G}_{1}, \mathbb{G}_{2}, \mathbb{G}_{3}$ respectively) are all disjoint. All three lists are initially instantiated with the identity. Note that it is not strictly necessary to instantiate the identity in $\mathbb{G}_{3}$ since it can be calculated using the other information provided. We precompute the representations of the randomness used for the key update, the $r_{i}^{\ominus}$ 's and $r_{i}^{\ominus}$ 's, but since the adversary does not have access to this list of elements, this does not effect the game.

The adversary $\mathbb{A}$ outputs a bit $b^{\prime}$ and is said to have won if:

1. $\mathcal{F}_{i}^{l}=\mathcal{F}_{j}^{l}$ for $l \in\{1,2,3\}$ and $i \neq j$

2. $b^{\prime}=b$

The first case corresponds to the adversary being able to create two polynomials which evaluate to the same value. If this occurs then a single group element has two representations. The adversary is said to have won because the simulation has been broken. The second case corresponds to the adversary being able to distinguish which world they are in. The only way (beyond guessing) that an adversary can distinguish between the two worlds is if they can construct a polynomial $\mathcal{F}_{i}^{3}$ such that $\mathcal{F}_{i}^{3}-\mathcal{K} \cdot \mathcal{H}=0$. Where $\mathcal{H}$ is the indeterminant corresponding to the hash of an $X$ that has not been sent to the PRF oracle. This corresponds to the adversary winning the PRLF game. We first bound the chance of a collision and then go on to bound the chance of winning the game.

All polynomials originally in $\mathcal{L}_{1}, \mathcal{L}_{2}, \mathcal{L}_{3}$ are of degree one and the only operation that increases the degree is the pairing operation which can only be called on elements in $\mathbb{G}_{1}, \mathbb{G}_{2}$. This means that degree two polynomials can be in $\mathbb{G}_{3}$ but not the other two lists (since there is no way to get an element of $\mathbb{G}_{3}$ into either of the other two groups). Hence, by the Schwartz-Zippel lemma, the probability of two (nonzero) polynomials evaluating to the same value is $\frac{2}{p}$. Since there are, at most, $\gamma$ polynomials, there are up to $\left(\begin{array}{l}\gamma \\ 2\end{array}\right)=\frac{\gamma(\gamma-1)}{2}$ pairs of polynomials that could collide and thus the probability of any two polynomials colliding is at most $\frac{\gamma(\gamma-1)}{p}$.

Without loss of generality, we will now only look at leakage in the target group $\mathbb{G}_{3}$ since any element from $\mathbb{G}_{1}, \mathbb{G}_{2}$ calculated by the leakage can be transferred over to $\mathbb{G}_{3}$ using a pairing, with the corresponding generator, and any elements known to the adversary can easily be embedded into the leakage by the adversary as required. Since, any element which can be leaked upon from $\mathcal{L}_{1}$ or $\mathcal{L}_{2}$ can be leaked on from $\mathcal{L}_{3}$, by transferring the element to $\mathbb{G}_{3}$ with a pairing, this does not weaken the adversary.

While only the leakage from $\mathcal{L}_{3}$ needs to be considered, due to the OCLI assumption $F^{\ominus}, F^{\ominus}$ and $F^{\ominus}$ will have access to different secret information and, due to the randomness used to update the key, each iteration will have access to different secret information. The lists $\mathcal{L}_{i}^{\ominus}, \mathcal{L}_{i}^{\ominus}$ and $\mathcal{L}_{i}^{\ominus}$ will represent all elements which can be calculated by $L_{i}^{\ominus}, L_{i}^{\ominus}$ and $L_{i}^{\ominus}$ respectively.

Let $\mathcal{L}_{i}^{\ominus}$ be the set of elements that could be computed by the leakage function $L_{i}^{\ominus}$. Then, utilising the leakage functions from the theorem statement:

$$
\mathcal{L}_{i}^{\ominus}=\left\{A \cdot \mathcal{S}_{i}^{\ominus}+B \cdot \mathcal{R}_{i+1}^{\ominus}+C\right\},
$$


where $A, B \in \mathcal{F}_{p}\left[\left\{\mathcal{H}_{j}\right\}_{j=1}^{q_{H}},\left\{\mathcal{V}_{j}\right\}_{j=1}^{2 q_{O}}\right]$ and $C \in \mathcal{F}_{p}\left[\mathcal{K}\left\{\mathcal{H}_{j}\right\}_{j=1}^{i-1},\left\{\mathcal{H}_{j}\right\}_{j=1}^{q_{H}},\left\{\mathcal{Y}_{j}\right\}_{j=1}^{q_{R}},\left\{\mathcal{U}_{j}\right\}_{j=1}^{2 q_{O}}\right.$, $\left.\left\{\mathcal{V}_{i}\right\}_{i=1}^{2 q_{O}},\left\{\mathcal{W}_{i}\right\}_{i=1}^{q_{O}}\right]$ and $\mathcal{S}_{i}^{\Theta}$ denotes $\sum_{j=0}^{i} \mathcal{R}_{j}^{\Theta}$ (corresponding to the definition of $S_{i}^{\boldsymbol{\Theta}}$ in the PRLF).

Let $\mathcal{L}_{i}^{\ominus}$ be the set of elements that could be computed by the leakage function $L_{i}^{\ominus}$. Therefore,

$$
\mathcal{L}_{i}^{\ominus}=\left\{A \cdot \mathcal{S}_{i}^{\ominus}+B \cdot \mathcal{R}_{i+1}^{\ominus}+C\right\}
$$

where $\mathcal{S}_{i}^{\ominus}$ denotes $\sum_{j=0}^{i} \mathcal{R}_{j}^{\ominus}$ (corresponding to the definition of $S_{i}^{\ominus}$ in the PRLF).

Let $\mathcal{L}_{i}^{\ominus}$ be the set of elements that could be computed by the leakage function $L_{i}^{\ominus}$. Therefore,

$$
\mathcal{L}_{i}^{\ominus}=\left\{A \cdot \mathcal{S}_{i}^{\ominus}+B^{\ominus} \cdot \mathcal{R}_{i+1}^{\ominus}+B^{\ominus} \cdot \mathcal{R}_{i+1}^{\ominus}+C+d^{\ominus} \cdot \mathcal{S}_{i}^{\ominus} \cdot \mathcal{H}_{i}+d^{\ominus} \cdot \mathcal{S}_{i}^{\ominus} \cdot \mathcal{H}_{i}\right\}
$$

where $d^{\ominus}, d^{\ominus} \in \mathcal{F}_{p}, B^{\ominus}, B^{\ominus} \in \mathcal{F}_{p}\left[\left\{\mathcal{H}_{j}\right\}_{j=1}^{q_{H}},\left\{\mathcal{V}_{j}\right\}_{j=1}^{2 q_{O}}\right]$ and $\mathcal{S}_{i}^{\ominus}$ denotes $\mathcal{K}-\sum_{j=0}^{i}\left(\mathcal{R}_{j}^{\ominus}+\mathcal{R}_{j}^{\ominus}\right)$ (corresponding to the definition of $S_{i}^{\ominus}$ in the PRLF). Without loss of generality we will assume that it PRF call maps to $\mathcal{H}_{i}$.

The adversary can win if they can leak $\mathcal{H} \cdot \mathcal{K}$ where $\mathcal{H}$ corresponds to some unqueried value $X$. However, there is no such linear combination within the leakage sets that allows this to be possible.

To bound the leakage per element, we will only consider leakage functions which contains at least one unknown group element. Since, while completely known elements can be leaked on multiple times, they do not give the adversary any new information. By showing that each element can only leak a bounded number of times ( 4 times), the adversary cannot learn any group elements which they would not be able to learn when leakage is not involved and thus the advantage will be increased by at most the number of bits the adversary can learn.

- $\mathcal{S}_{i}^{\ominus}$ can be leaked on twice: once in $\mathcal{L}_{i-1}^{\ominus}$ since $S_{i-1}^{\ominus}$ is passed in and $r_{i}^{\ominus}$ is generated internally (represented by the polynomials $\mathcal{S}_{i-1}^{\ominus}$ and $\mathcal{R}_{i}^{\ominus}$ ), and once in $\mathcal{L}_{i}^{\ominus}$ since it is passed in.

- $S_{i}^{\ominus}$ can be leaked on twice: once in $\mathcal{L}_{i-1}^{\ominus}$ since $S_{i-1}^{\ominus}$ is passed in and $r_{i}^{\ominus}$ is generated internally (represented by the polynomials $\mathcal{S}_{i-1}^{\ominus}$ and $\mathcal{R}_{i}^{\ominus}$ ), and once in $\mathcal{L}_{i}^{\ominus}$ since it is passed in.

- $\mathcal{S}_{i}^{\ominus}$ can be leaked on twice: once each in $\mathcal{L}_{i-1}^{\ominus}$ and $\mathcal{L}_{i}^{\ominus}$ due to a similar argument as above.

- $\mathcal{R}_{i+1}^{\ominus}$ can be leaked on twice: once each in $\mathcal{L}_{i}^{\ominus}$ and $\mathcal{L}_{i}^{\ominus}$ since it is generated in $F^{\ominus}$ and then passed into $F^{\ominus}$ on the $i^{\text {th }}$ iteration.

- $\mathcal{R}_{i+1}^{\ominus}$ can be leaked on twice: once each in $\mathcal{L}_{i}^{\ominus}$ and $\mathcal{L}_{i}^{\ominus}$ since it is generated in $F^{\ominus}$ and then passed into $F^{\ominus}$ on the $i^{\text {th }}$ iteration.

$-S_{i}^{\ominus} \cdot \mathcal{H}_{i}$, the intermediated state, can be leaked on 4 times: once in each of $\mathcal{L}_{i-1}^{\ominus}, \mathcal{L}_{i}^{\ominus}, \mathcal{L}_{i-1}^{\ominus}$, and $\mathcal{L}_{i}^{\ominus}$ using the argument above for calculating the next share and the fact for input $X_{i}$, on the $i^{\text {th }}$ tag call the intermediate state is generated in $F^{\ominus}$ and passed into $F^{\ominus}$. To leak on this four times will require querying the same input twice.

$-S_{i}^{\ominus} \cdot \mathcal{H}_{i}$, the intermediated state, can be leaked on 4 times: once in each of; $\mathcal{L}_{i-1}^{\ominus}, \mathcal{L}_{i}^{\ominus}, \mathcal{L}_{i-1}^{\ominus}$, and $\mathcal{L}_{i}^{\ominus}$ using the argument above for calculating the next share and the fact for input $X_{i}$, on the $i^{\text {th }}$ tag call the intermediate state is generated in $F^{\ominus}$ and passed into $F^{\ominus}$. To leak on this four times will require querying the same input twice.

Since each element can only be leaked on at most four times, the adversary can only learn up to $4 \cdot \lambda$ bits of information per unknown group element. Therefore, the adversary's advantage can be at most $2^{4 \cdot \lambda}$ times the advantage of playing the standard non-leakage game. This results in the bound given in the theorem statement. 


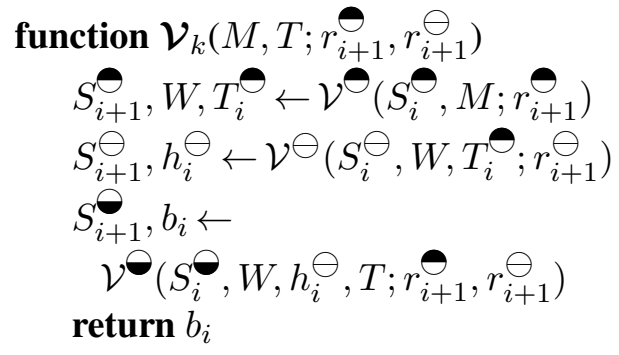

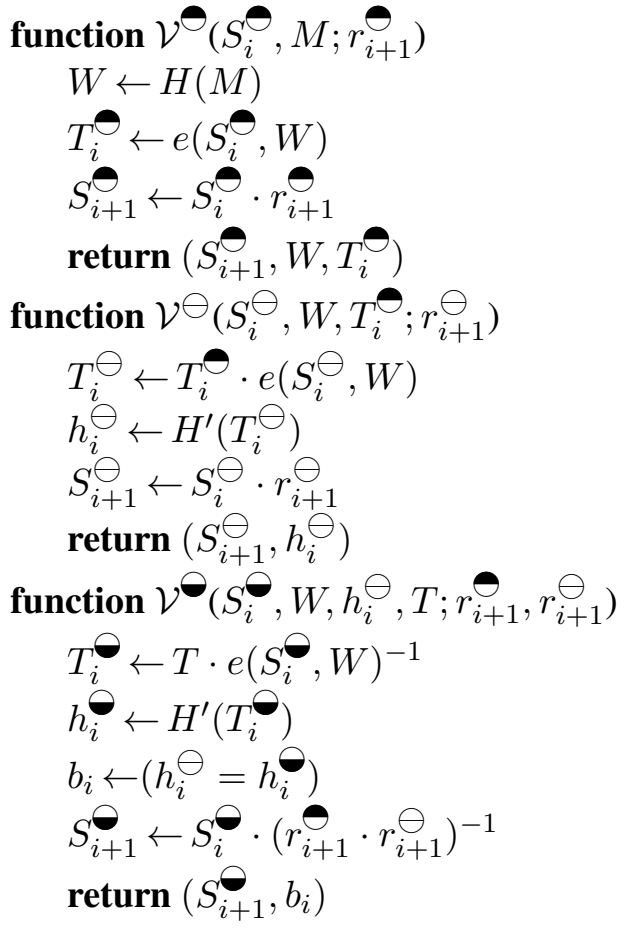

Fig. 16: A secure implementation $\mathcal{V}$. The two-share version $\mathcal{V}_{2}$ merges $\mathcal{V}^{\ominus}$ and $\mathcal{V}^{\ominus}$

\section{A.4 A fully Leakage Resilient MAC}

The MOSW verification routine uses the classical method of recomputing a tag to verify correctness. However, this is not secure under verification leakage, since an attacker may leak (functions of) the candidate tag. Thus providing a MAC secure under verification leakage was left as an open problem.

In this work we answer this, providing a secure verification implementation $\mathcal{V}$ in Figure 16. Instead of calculating a candidate tag, we invert the final pairing step, and surrounding the comparison in a random oracle query, meaning the candidate tag is never available to the adversary.

For simplicity, consider the (also secure) two share version $\mathcal{V}_{2}$. If $T$ is a valid tag on message $M$ under key $k=S^{\ominus} \cdot S^{\ominus}$, then $T=e(k, H(M))$. Instead of calculating $T^{\prime}=e\left(S^{\boldsymbol{\bullet}}, H(M)\right) \cdot e\left(S^{\bullet}, H(M)\right)$ and testing whether $T^{\prime}=T$, we calculate the two pairings and check whether $e\left(S^{\ominus}, H(M)\right)=$ $T \cdot e\left(S^{\ominus}, H(M)\right)^{-1}$. However, rather than doing this final comparison directly, we enclose each side in a random oracle call to ensure the adversary can never leak on both parts of the tag in unison. This results in the comparision of $H^{\prime}\left(e\left(S^{\ominus}, H(M)\right)\right)=H^{\prime}\left(T \cdot e\left(S^{\ominus}, H(M)\right)^{-1}\right)$ This allows us to recover security even under a large set of verification leakage functions, meaning both $\left(\mathcal{T}_{2}, \mathcal{V}_{2}\right)$ and $(\mathcal{T}, \mathcal{V})$ define secure LMAC implementations under the OCLI assumption.

For our overall implementation, we require that the tagging algorithm is a LPRF, and so use the three share variant $\mathcal{V}$, since key updating agrees with $\mathcal{T}$. To prove security under OCLI, we define the verification leakage set $\mathcal{L}_{\vee}$ to be the set of functions of the form:

$$
\begin{aligned}
& L_{V}\left(\left(S^{\ominus}, S^{\ominus}\right), M, T ;\left(r^{\ominus}, r^{\ominus}\right)\right) \\
& \quad=\left(L_{V}^{\ominus}\left(S^{\ominus}, M ; r^{\ominus}\right), L_{V}^{\ominus}\left(S^{\ominus}, H(M), T^{\ominus} ; r^{\ominus}\right), L_{V}^{\ominus}\left(S^{\ominus}, H(M), T, T^{\ominus} ; r^{\ominus}, r^{\ominus}\right)\right)
\end{aligned}
$$

where $L^{\ominus}, L^{\ominus}$ and $L^{\ominus}$ each output $\lambda$ bits, $S^{\ominus}, S^{\ominus}$ and $S^{\ominus}$ is the secret information used by each share, $r^{\ominus}, r^{\ominus}$ the randomness, and $T_{i}^{\ominus}, T_{i}^{\ominus}$ the information passed between the shares. This allows us to state the following theorem, reducing to the security in the leakage-free case, shown secure by MOSW.

Theorem 9. Let $\mathbb{A}$ be an adversary against the $\left(\mathcal{L}_{\mathrm{T}}, \mathcal{L}_{\mathrm{V}}\right)$-sEUF-CMLA security of $(\mathcal{T}, \mathcal{V})$ in the generic group model, for $H^{\prime}$ a random permuation. Then, there exists an adversary $\mathbb{A}_{\mathrm{MAC}}$ (of similar complexity 
to $\mathbb{A})$ against the sEUF-CMLA security of $(\mathcal{T}, \mathcal{V})$ such that

$$
\mathbf{A d v}_{\mathcal{T}, \mathcal{V} ; \mathcal{L}_{\mathrm{T}}, \mathcal{L}_{V}}^{\mathrm{sEU}}(\mathbb{A}) \leq 2^{4 \cdot \lambda} \cdot \mathbf{A d v}_{\mathcal{T}, \mathcal{V}}^{\mathrm{sEUF}-\mathrm{CMA}}\left(\mathbb{A}_{\mathrm{MAC}}\right)+\frac{\gamma(\gamma-1)}{p}
$$

where $\gamma$ is the total number of group elements, $p$ is the size of the group and $\lambda$ is the amount of leakage output by each of the three parts of the leakage function.

Proof. In this proof we use the same initial setup of representing group elements as the LPRF proof, with the same indeterminants. In this game the adversary $\mathbb{A}$ outputs the pair $(M, T)$ and is said to have won if:

1. $\mathcal{F}_{i}^{l}=\mathcal{F}_{j}^{l}$ for $l \in\{1,2,3\}$ and $i \neq j$

2. $\mathcal{K} \cdot \mathcal{H}^{*}-\mathcal{T}=0$ where $\mathcal{H}^{*}$ is the indeterminant corresponding to the hash of $M, \mathcal{T}$ is the corresponding polynomial for $T$ and $T$ was not output from the tag oracle

The first case corresponds to the adversary being able to create two polynomials which evaluate to the same value. Two distinct polynomials evaluating to the same value, means that a single group element has two distinct representations. This breaks the simulation and therefore the adversary is said to have won the game. As previously this can be bounded as $\gamma(\gamma-1) / p$. The second case corresponds to the adversary being able to create a forgery on the MAC. The polynomial given in the second case evaluating to zero implies that the adversary output a valid forgery for the MAC. Therefore, they have won the sEUF-CMLA game.

As before we consider what the $i^{\text {th }}$ leakage function can leak on. We now have to consider leakage sets for both tag and verify queries. The tagging query leakage is the same as the leakage for the LPRF above but is recapped below.

$$
\begin{gathered}
\mathcal{L}_{i}^{\ominus}=\left\{A \cdot \mathcal{S}_{i}^{\ominus}+B \cdot \mathcal{R}_{i+1}^{\ominus}+C\right\} \\
\mathcal{L}_{i}^{\ominus}=\left\{A \cdot \mathcal{S}_{i}^{\ominus}+B \cdot \mathcal{R}_{i+1}^{\ominus}+C\right\} \\
\mathcal{L}_{i}^{\ominus}=\left\{A \cdot \mathcal{S}_{i}^{\ominus}+\mathcal{B}^{\ominus} \cdot \mathcal{R}_{i+1}^{\ominus}+\mathcal{B}^{\ominus} \cdot \mathcal{R}_{i+1}^{\ominus}+C+d^{\ominus} \cdot \mathcal{S}_{i}^{\ominus} \cdot \mathcal{H}_{i}+d^{\ominus} \cdot \mathcal{S}_{i}^{\ominus} \cdot \mathcal{H}_{i}\right\}
\end{gathered}
$$

We then give the leakage sets for verify. It is important to note the extra element in the $\mathcal{J}_{i}^{\ominus}$ set (which was added on in the $\mathcal{L}_{i}^{\ominus}$ set). This cannot be added on in this set because it was passed through the second hash function $H^{\prime}$ first and therefore is no longer in the group.

$$
\begin{gathered}
\mathcal{J}_{i}^{\ominus}=\left\{A \cdot \mathcal{S}_{i}^{\ominus}+B \cdot \mathcal{R}_{i+1}^{\ominus}+C\right\} \\
\mathcal{J}_{i}^{\ominus}=\left\{A \cdot \mathcal{S}_{i}^{\ominus}+B \cdot \mathcal{R}_{i+1}^{\ominus}+C+d \cdot \mathcal{S}_{i}^{\ominus} \cdot \mathcal{H}_{i}\right\} \\
\mathcal{J}_{i}^{\ominus}=\left\{A \cdot \mathcal{S}_{i}^{\ominus}+\mathcal{B}^{\ominus} \cdot \mathcal{R}_{i+1}^{\ominus}+\mathcal{B}^{\ominus} \cdot \mathcal{R}_{i+1}^{\ominus}+C, d^{\ominus} \cdot \mathcal{S}_{i}^{\ominus} \cdot \mathcal{H}_{i}+d^{\ominus} \cdot \mathcal{S}_{i}^{\ominus} \cdot \mathcal{H}_{i}\right\}
\end{gathered}
$$

The adversary can win if they can leak $\mathcal{H} \cdot \mathcal{K}$ where $\mathcal{H}$ corresponds to some unqueried value $X$. However, there is no such linear combination within the leakage sets that allows this to be possible.

To bound the leakage per element, we will only consider leakage functions which contains at least one unknown group element. Since, while completely known elements can be leaked on multiple times, they do not give the adversary any new information. By showing that each element can only leak a 
bounded number of times, the adversary cannot learn any group elements which they would not be able to learn when leakage is not involved and thus the advantage will be increased by at most the number of bits the adversary can learn.

A similar approach, to the one given for the LPRF, can be used to show that each (unknown) element can be leakage on at most four times. Since each element can only be leaked on, at most, four times, the adversary can only learn up to $4 \cdot \lambda$ bits of information per unknown group element. Therefore, the adversary's advantage can be at most $2^{4 \cdot \lambda}$ times the advantage of playing the standard non-leakage game. This results in the bound given in the theorem statement.

As shown by Martin et al. [36] the security of the MAC without leakage takes the same form as that of the PRF, except it reduces to the computational variant of the Diffie-Hellman style problem, instead of the decisional one. However, since then computational problem is at least as hard as the decisional variant, the MAC security is bounded by the PRF security (In fact, in the GGM they are identical, since the decisional problem is no easier than the computational one).

\section{A.5 nLAE and mrLAE Constructions in the Generic Group Model}

Collecting together the bounds for each component with the security of the A5 and SIVAT composition mechanisms, we are able to give the first provably secure leakage resilient AE schemes. Carefully counting the number of generic group queries $\gamma$ made by the different elements of the construction, we find that $\gamma \leq 3 g+27 q+14 \sigma$, where $g$ is the total number of direct queries the adversary makes to the generic group or random oracle, $q$ the number of AE oracle queries (honest or challenge) of total $\sigma$ blocks. This is formally stated as Theorem 10 for A5, and was already given as Theorem 5 for the SIVAT case.

Theorem 10. Let $\left(\mathcal{L}_{\mathrm{ivE}}, \mathcal{L}_{\mathrm{ivD}}, \mathcal{L}_{\mathrm{T}}, \mathcal{L}_{\mathrm{V}}, \mathcal{L}_{\mathrm{F}}\right)$ be leakage sets as defined within this section, parametrised by some $\lambda$, and define $\left(\mathcal{L}_{\text {Enc }}, \mathcal{L}_{\text {Dec }}\right)$ as in Section 5.1. Then for any adversary $\mathbb{A}$ against the $\left(\mathcal{L}_{\text {Enc }}, \mathcal{L}_{\text {Dec }}\right)$ $n L A E$ security of $\mathrm{A} 5[\mathrm{iv} \mathcal{E}, \operatorname{iv} \mathcal{D} ; \mathcal{F} ; \mathcal{T}, \mathcal{V}]$ making at most q queries to his oracles totalling up to $\sigma$ blocks and making $g$ direct queries to the generic group oracle (including the complexity of all chosen leakage queries)

$$
\begin{aligned}
\operatorname{Adv}_{\mathrm{A} 5 ; \mathcal{L}_{\text {Enc }}, \mathcal{L}_{\mathrm{Dec}}}^{\mathrm{nLAE}}(\mathbb{A}) & \leq \frac{3}{4 p}\left(2^{4 \lambda} \cdot \sigma^{2} \cdot \gamma^{2}+8 \cdot \gamma^{2}\right) \\
& \leq \frac{7}{p}\left(2^{4 \lambda} \cdot \sigma^{2} \cdot(g+9 q+5 \sigma)^{2}+8(g+9 q+5 \sigma)^{2}\right),
\end{aligned}
$$

where $\gamma$ is the total number of generic group elements generated.

Proof (Simultaneously proving Theorem 5). Without loss of generality, assume $\mathbb{A}$ makes the maximum number of queries allowed. Now, let us begin by collecting the previous theorems to generate the first bound, given in terms of $\gamma$.

By Theorem 1, there exist adversaries $\mathbb{A}_{\mathrm{CPA}}, \mathbb{A}_{\mathrm{PRF}}$ and $\mathbb{A}_{\mathrm{MAC}}$ each making $q$ queries totalling $\sigma$ blocks such that

$$
\begin{aligned}
& \operatorname{Adv}_{\mathrm{A} 5 ; \mathcal{L}_{\text {Enc }}, \mathcal{L}_{\text {Dec }}}^{\mathrm{nLAE}}(\mathbb{A}) \leq \mathbf{A d v}_{\mathcal{E}, \mathcal{D} ; \mathcal{L}_{\text {ive }}, \mathcal{L}_{\text {iv }}}^{\mathrm{IND}}\left(\mathbb{A}_{\mathrm{CPA}}\right)+2 \cdot \mathbf{A d v}_{\mathcal{F} ; \mathcal{L}_{\mathrm{F}}}^{\mathrm{LPRF}}\left(\mathbb{A}_{\mathrm{PRF}}\right) \\
& +2 \cdot \mathbf{A d v}_{\mathcal{T}, \mathcal{V} ; \mathcal{L}_{\mathrm{T}}, \mathcal{L}_{\mathrm{V}}}^{\mathrm{sEUF}}\left(\mathbb{A}_{\mathrm{MAC}}\right) .
\end{aligned}
$$

By Theorem 4, there exists a PRLF adversary $\overline{\mathbb{A}_{\mathrm{CPA}}}$ making $\sigma$ single-block queries such that

$$
\mathbf{A d v}_{\mathbf{i v} \mathcal{E}, \text { iv } \mathcal{D} ; \mathcal{L}_{\text {ive }}, \mathcal{L}_{\mathrm{ivD}}}^{\mathrm{IND}}\left(\mathbb{A}_{\mathrm{CPA}}\right) \leq 2 \cdot \mathbf{A d v}_{\mathcal{F} ; \mathcal{L}_{\mathrm{F}}}^{\mathrm{PRLF}}\left(\overline{\mathbb{A}_{\mathrm{CPA}}}\right)+\frac{3}{4} \cdot \frac{\sigma^{2}}{p}
$$

By Theorem 8 , for any adversary $\mathbb{B}$ there exists an adversary $f[\mathbb{B}]$ making the same number and length of queries such that

$$
\operatorname{Adv}_{\mathcal{T} ; \mathcal{L}_{\boldsymbol{T}}}^{\mathrm{LPRF}}(\mathbb{B}) \leq 2^{4 \lambda} \cdot \mathbf{A d v}_{\mathcal{T}}^{\mathrm{PRF}}(f[\mathbb{B}])+\frac{\gamma(\gamma-1)}{p}
$$


By Theorem 9, there exists $\overline{\mathbb{A}_{\mathrm{MAC}}}$ making $q$ queries of total $\sigma$ blocks such that

$$
\mathbf{A d v}_{\mathcal{T}, \mathcal{V} ; \mathcal{L}_{\mathrm{T}}, \mathcal{L}_{V}}^{\mathrm{sEUF}}\left(\mathbb{A}_{\mathrm{MAC}}\right) \leq 2^{4 \lambda} \cdot \mathbf{A d v}_{\mathcal{T}, \mathcal{V}}^{\mathrm{sEUF}-\mathrm{CMA}}\left(\overline{\mathbb{A}_{\mathrm{MAC}}}\right)+\frac{\gamma(\gamma-1)}{p} .
$$

Collecting these together,

$$
\begin{aligned}
\operatorname{Adv}_{\mathrm{A} 5 ; \mathcal{L}_{\mathrm{Enc}}, \mathcal{L}_{\mathrm{Dec}}}^{\mathrm{nLAE}}(\mathbb{A}) \leq & \left(2 \cdot 2^{4 \lambda} \cdot \mathbf{A d v}_{\mathcal{T}}^{\mathrm{PRF}}\left(f\left[\overline{\mathbb{A}_{\mathrm{CPA}}}\right]\right)+2 \cdot \frac{\gamma(\gamma-1)}{p}+\frac{3 \sigma^{2}}{4 p}\right) \\
& +2 \cdot\left(2^{4 \lambda} \cdot \mathbf{A d v}_{\mathcal{T}}^{\mathrm{PRF}}\left(f\left[\mathbb{A}_{\mathrm{PRF}}\right]\right)+\frac{\gamma(\gamma-1)}{p}\right) \\
& +2 \cdot\left(2^{4 \lambda} \cdot \mathbf{A d v}_{\mathcal{T}, \mathcal{V}}^{\mathrm{sEUF}-\mathrm{CMA}}\left(\overline{\mathbb{A}_{\mathrm{MAC}}}\right)+\frac{\gamma(\gamma-1)}{p}\right)
\end{aligned}
$$

Using the fact that the sEUF-CMLA advantage is upper bounded by the PRF advantage, each of these advantages is upper bounded by the maximum advantage of any adversary against the PRF querying a total of $\sigma$ blocks. By Theorem 7, for any particular adversary $\mathbb{A}$, this is bounded by

$$
\mathbf{A d v}_{\mathcal{T}}^{\mathrm{PRF}}(\mathbb{B}) \leq\left(\frac{\sigma}{2}\right)^{2} \cdot \mathbf{A d v}_{\mathbb{B} \mathbb{G}}^{\mathrm{DTBDH}}(\mathbb{B}) \leq \frac{\sigma^{2}}{4} \cdot \frac{\gamma(\gamma-1)}{2 p},
$$

where the second bound uses Theorem 6 to explicitly bound the hardness of DTBDH in the generic group model.

Thus overall,

$$
\begin{aligned}
\operatorname{Adv}_{\mathrm{A} 5 ; \mathcal{L}_{\text {Enc }}, \mathcal{L}_{\text {Dec }}}^{\mathrm{nLAE}}(\mathbb{A}) & \leq 6\left(2^{4 \lambda} \cdot \mathbf{A d v}_{\mathcal{T}}^{\mathrm{PRF}}(\sigma)+\frac{\gamma(\gamma-1)}{p}\right)+\frac{3 \sigma^{2}}{4 p} \\
& \leq \frac{3}{4 p}\left(2^{4 \lambda} \cdot \sigma^{2} \cdot \gamma(\gamma-1)+8 \gamma(\gamma-1)+\sigma^{2}\right) \\
& \leq \frac{3}{4 p}\left(2^{4 \lambda} \cdot \sigma^{2} \cdot \gamma^{2}+8 \cdot \gamma^{2}\right),
\end{aligned}
$$

which is the first bound in the theorem statement. For the final inequality, we have merged the first and last terms using $\sigma^{2}(\gamma(\gamma-1)+1) \leq \sigma^{2} \gamma^{2}$.

Thus we are left to bound $\gamma$, the total number of generic group elements constructed by the adversary. This is just a tedious calculation, and proceeds as follows. First we note that for each query to the oracle, inputting just arbitrary strings of their own choice they may discover said strings were valid and thus learn three new elements. Thus the $g$ oracle queries made yield up to $3 g$ group elements.

Consider now the evaluations of the tagging or PRF function $\mathcal{T}$. Assuming all variables are new, two new elements $r$ must be sampled (2, prior to calling), one hash value (1 operation), 3 partial outputs (3 pairings), 1 actual output ( 2 operations), and three updated key shares computed ( 5 operations). Thus in total there are 13 new elements for each call to $\mathcal{T}$.

Suppose the adversary makes $q_{e}$ encryption queries of total $\sigma_{e}$ blocks, and to decryption define $q_{d}, \sigma_{d}$ equivalently. In total across their encryption queries they make $q_{e}$ PRF queries creating the IVs, $\sigma_{e}$ generating the stream, and $q_{e}$ on tagging queries, along with $\sigma_{e}$ new elements from the chaining mode. Finally, each encryption query may confirm that a candidate string does indeed correspond to a group element, thus creating an additional $q_{e}$ group elements. Thus the total number of elements created during encryption is $13\left(2 q_{e}+\sigma_{e}\right)+\sigma_{e}+q_{e}=14\left(2 q_{e}+\sigma_{e}\right)-q_{e}$.

On decryption things are slightly more complicated. Consider a PRF call on an input that has already been queried. Such a query repeats the hash call, and the final ciphertext call, thus taking 11 new elements. Verification costs two hash calls more than tagging, but recall invalid queries stop processing at this point. So, invalid queries (which have new content) cost 15 elements, plus possibly proving that $q_{d}$ candidate strings were indeed elements. Valid queries do not create so many new elements, but do proceed to complete the whole encryption routine. Thus a valid query requires just 11 new elements per PRF query, but makes as many as the encryption routine. In total then, a valid query of length $\sigma_{v}$ creates $11\left(2+\sigma_{v}\right)$. Thus the number of elements created on a decryption query is maximised by making valid queries, creating a total of $11\left(2 q_{d}+\sigma_{d}\right)$ elements.

Overall then, the bound is maximised by making just encryption queries, meaning

$$
\gamma \leq 3 g+14(2 q+\sigma)-q=3 g+27 q+14 \sigma \leq 3(g+9 q+5 \sigma) .
$$

Substituting this into the advantage, and bounding $\left(3^{2}\right) * 3 / 4=27 / 4<7$ completes the proof. 
If Shrimpton and Terashima's [55] (weaker) notion were considered to be sufficient, where "recovery information" is not required to be random and hence the LMAC's tagging algorithm need not also be a LPRF. This would lead to a minor improvement in the overall bound, reducing the constant factor slightly. Similar improvements can be made by tightening the analysis throughout. 